

\section{DISCLAIMER}

This report was prepared as an account of work sponsored by an agency of the United States Government. Neither the United States Government nor any agency Thereof, nor any of their employees, makes any warranty, express or implied, or assumes any legal liability or responsibility for the accuracy, completeness, or usefulness of any information, apparatus, product, or process disclosed, or represents that its use would not infringe privately owned rights. Reference herein to any specific commercial product, process, or service by trade name, trademark, manufacturer, or otherwise does not necessarily constitute or imply its endorsement, recommendation, or favoring by the United States Government or any agency thereof. The views and opinions of authors expressed herein do not necessarily state or reflect those of the United States Government or any agency thereof. 


\section{DISCLAIMER}

Portions of this document may be illegible in electronic image products. Images are produced from the best available original document. 


\title{
THERMONUCLEAR BURN IN WALL-CONFINED PLASMAS
}

\author{
Lubomyra Nadia Kmetyk
}

\section{Submitted in partial fulfillment of the requirements for the degree of Doctor of Engineering Science}

in the School of Engineering and Applied Science

COLUMBIA UNIVERSITY

1979

This repon was prepared as an accnunt of work sputisured by the United States Covernment. Neither the United States nor the United States Department of Energy. nor any of their employees, Department of contractors, subcontrair employees, nor any of their any warranty, express or or their employees, makes lizbility or responsibs implied, or assumes any legal or usefulness of any in for the accuracy, completeness process diselosed, or information, apparatus, product or process disclosed, or epresents that its use would not
infringe privately owmed nights. 
ABSTRACT

The one-dimensional initial value problem describing a hot, dense, thermonuclear reacting plasma which is magnetically insulated and contained between cold metal wails has been studied by means of computational simulation. The resulting time evolution of the plasma temperature, number density and magnetic field profiles includes the effects of thermal conduction and radiation energy losses from the plasma, convection, and a detailed treatment of alpha particle heating, as well as ohmic heating and magnetic field diffusion.

The results are used to evaluate the net energy gains possible in a shock-heated, magnetically insulated, wall-confined fusion cycle. The energy multiplication is calculated for simple limiting cases and then in greater detail using one-dimensional time-dependent physics. The effects on the energy gain of various two-dimensional loss mechanisms, such as particle drifts and boundary layer instabilities, are estimated using modified forms of the general one-dimensional model. Energy multiplications of between 10 and 20 are calculated for a cylinder whose mean radius is less than one meter. Energy breakeven is found for $L=20 \mathrm{~cm}, E_{\text {in }}=1.9 \mathrm{MJ}$, at a power input of $8.8 \mathrm{TW}$. 


\section{ACKNOWLEDGEMENTS}

I would like to thank Professor Robert A. Gross for his help in selecting this thesis topic, and his continued assistance. Thanks are also due to Laura Howes, for an excellent typing job.

I am very grateful to the many people in Los Alamos who aided me with this project -- most notably Len Margolin for his sympathy and for many helpful discussions, Tom 01 iphant and Irv Lindemuth for their help with the plasma-wall study, and Alton Coulter and Hans Weertman for helping with the alpha particle modelling.

I would like to acknowledge the moral support of my parents, even when they had no idea what in the world I was trying to do.

Finally, "of course, my heartfelt thanks to Wally Green and Dan Speyer, who were always there with a shoulder for me to cry on whenever I needed one. 
TABLE OF CONTENTS

Page

Abstract

Acknowledgements

1. Introduction

2. Physics of a Magnetically Insulated, Thermonuclear

Reacting Plasma Contained Between Cold Walls

A. Assumptions and Discussion 14

B. Equations and Boundary Conditions . 23

C. Numerical Method of Solution 33

D. Results $\cdots 44$

3. Energy Gains in a Shock-heated Wal1-confined

Fusion Reactor

64

A. Definition of Energy Gain and Calculation of Maximum Possible Energy Gain

B. Optimization of Energy Gain in the Presence of Radiation Cooling

C. Energy Gains from the General One-dimensional Model

D. Energy Gains in the Absence of Alpha Particle Heating and the Presence of Enhanced Thermal 
4. Discussion and Summary

Appendices

I. Characteristic Times and Lengths 91

II. Alpha Particle Modelling $\quad \therefore \quad \therefore \quad 99$

A. Time Dependence 99

B. Spatial Dependence 108

III. Shock Heating - linear geometry 125

IV. System Parameters : 134

V. Nomenclature $\quad \therefore \quad 136$

References $\quad \ldots \quad \ldots$

List of Figures 144 


\section{Chapter 1: Introduction}

This is a study of the physical behavior of hot, dense, thermonuclear reacting plasma which is magnetically. insulated and confined by metal walls. The thesis consists of two major parts: (1) a computational, initial value problem, study of deuterium-tritium fusion reacting plasma which is brought into sudden contact with a cold metal wall; (2) an evaluation of the net energy that a shock-heated, magnetically insulated, wall-confined fusion cycle can produce. The computational study of the time evolution of the plasma temperature, number density and magnetic field profiles is described in chapter 2, and includes the effects of thermal conduction and radiation losses, plasma convection, alpha particle and Ohmic heating, and magnetic field diffusion. The plasma profiles thus calculated are then used in chapter 3 to estimate the potential energy gains in a shock-heated wall-confined fusion cycle for various initial conditions, and the results are discussed in chapter 4.

Wall confinement of thermonuclear plasmas has not been emphasized in the past primarily because raw (no magnetic insulation) plasma would be rapidly cooled through conduction losses by contact with the wall, and by charge exchange and radiation losses, so that the thermonuclear reaction would be rapidly quenched. The energy density of the plasma is much too small to cause serious damage to a solid wall for a modest number of exposures. Wall confinement of plasmas is employed in various fusion concepts such as imploding liners, gas blankets, the $\mathrm{CO}_{2}$ laser heated solenoid and the wall-confined magnetically insulated fusion system described here. Budker [1-1] has described the U.S.S.R. program developing a linear cylindrical plasma configuration, heated by 
an electron beam and confined by a cylindrical wall, with the radial heat conduction losses decreased by a transverse magnetic field and the logitudinal plasma loss retarded by a multimirror system. Braams [1-2] in the Netherlands and Lehnert [1-3] in Sweden are studying gas blankets insulating hot plasma from walls. In the United States, Sherwood [1-4] has considered very high density plasmas confined by a. magnetically imploded solid liner and Gross [1-5,1-6] is studying the concept of shock-heated wall-confined magnetically insulated fusion, which appears to have an advantage over more standard confinement concepts in that it lies between the small size but high power density requirements of inertial confinement and the low power density but large size extreme of magnetically confined systems.

The shock-heated wall-confined cycle has undergone a significant simplification since it was first proposed in 1972, and is shown schematically in its current form in figure 1. Two coaxial concentric metal cylinders with dielectric end walls form a cavity of height $h=L$, inner radius $r_{j}=f_{1} L$ and outer radius $r_{0}=f_{2} L$, which is filled with a 50-50 mixture of deuterium-tritium gas. A current is applied which flows down the cylinder axis and returns along the outer cylinder wall; this current creates an azimuthal magnetic field in the cavity chamber. As a voltage is rapidly applied across the radial cylinder gap the gas breaks down, and the resulting radial current sheet forms a magnetic piston, which drives a strong transverse magnetohydrodynamic shock wave ahead of it as it propagates down the length of the cavity, as shown in figure 2a. (This technique has been used to generate and study very strong shock waves $[1-7,1-8,1-9,1-10$ and 1-11].) The shock wave reflects from the opposite end wall as shown in figure $2 b$, and 
z

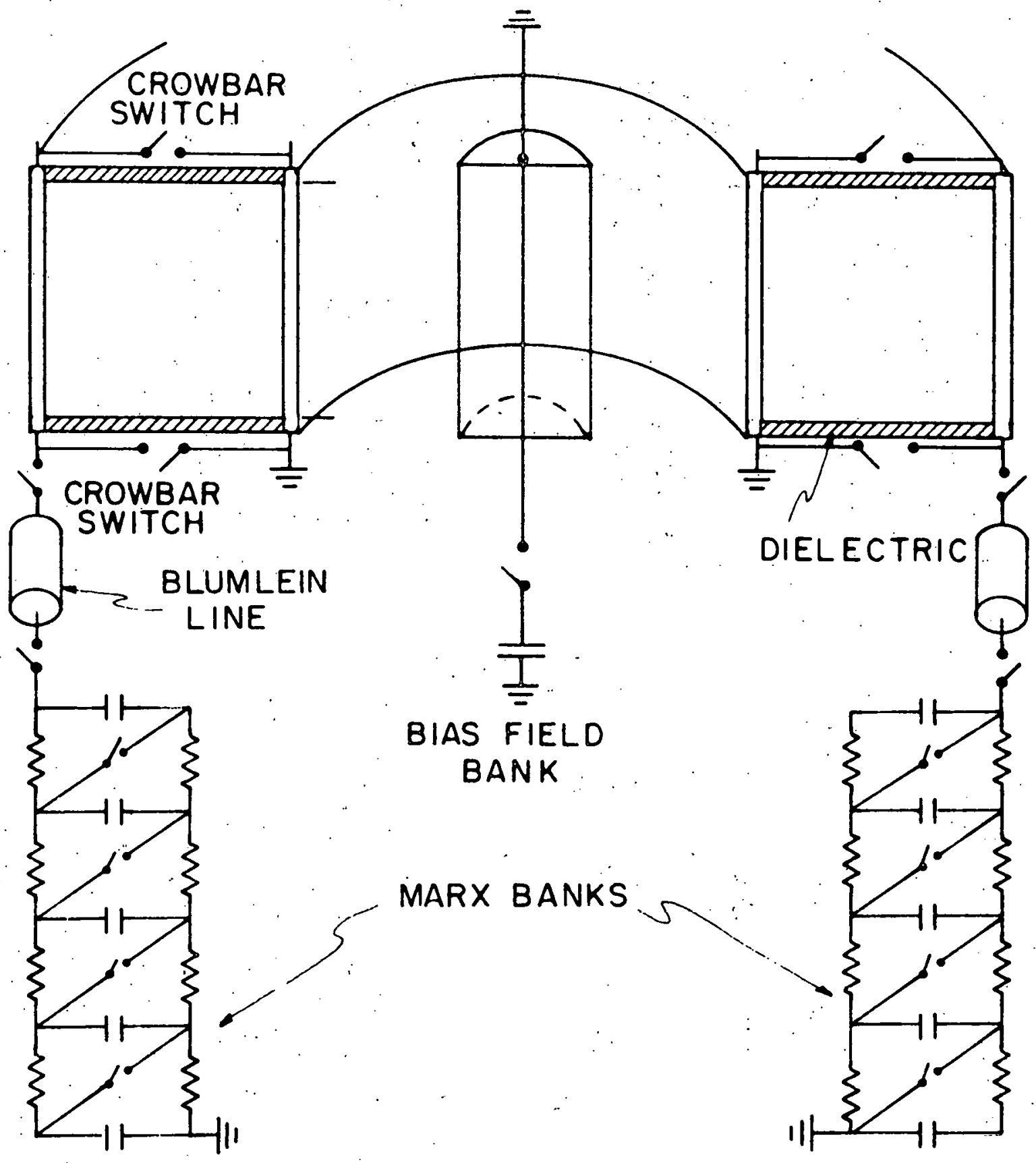

Figure 1 (chapter. 1) Schematic of a shock-heated wall-confined fusion reactor 

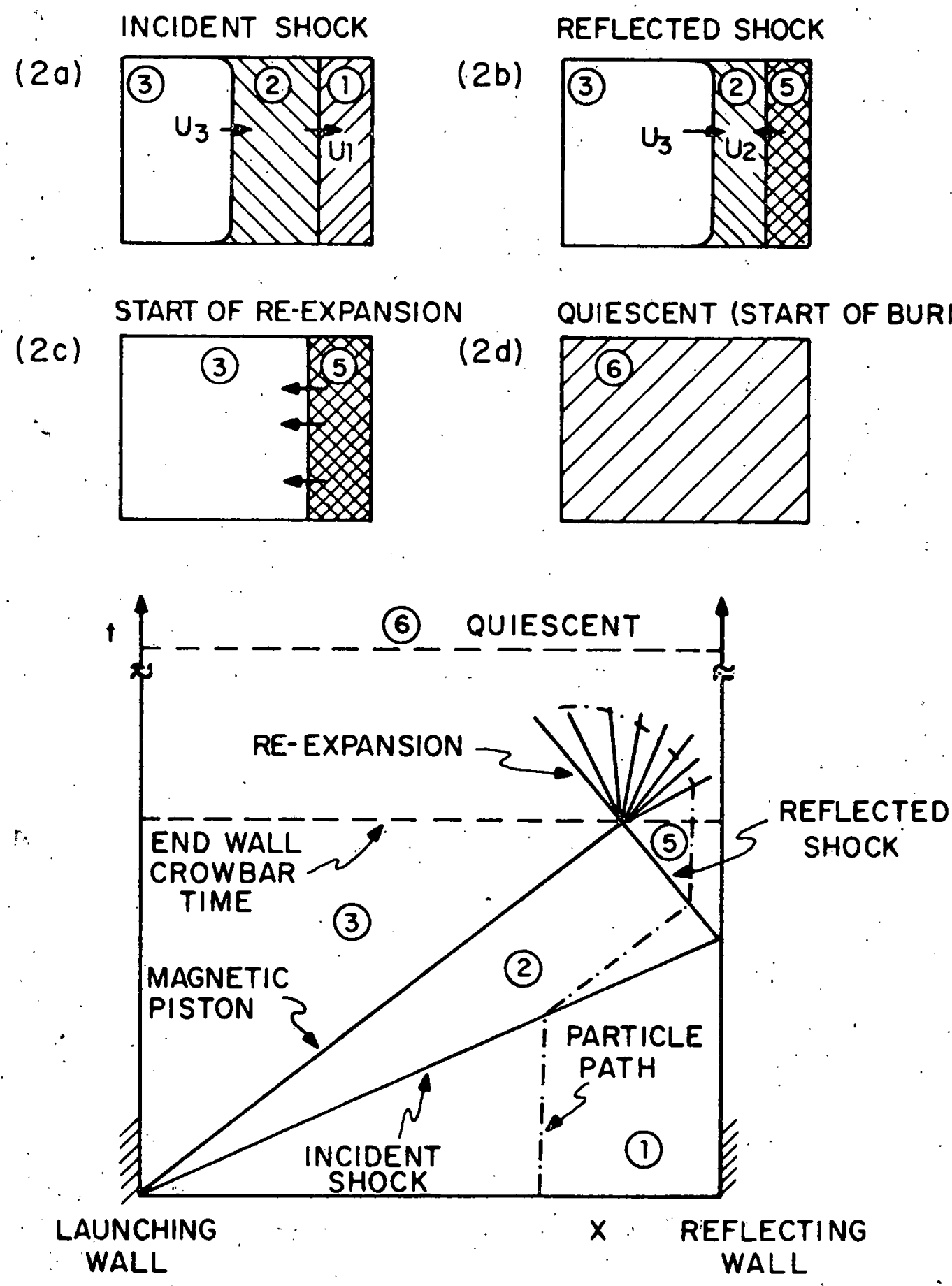

Figure 2 (chapter 1) Shock-heating and re-expansion 
further heats and compresses the plasma and imbedded magnetic field, until it reaches the vacuum interface between the magnetic piston and the plasma. At that instant, shown in figure $2 c$, the plasma consists of all the gas which has been swept up, heated by both the initial and reflected shocks, and compressed into about $10 \%$ of the cavity volume, while the rest of the coaxial chamber is filled with vacuum magnetic field.

Early conceptual designs [1-6] suggested using a magnetic dam or trap at one end of the system to contain the hot compressed plasma. This, however, required a large expenditure of energy, complicated and uncertain trapping physics, and large circulating power. In the more recent, simpler version of this cycle, plasma is allowed to re-expand and fill the entire cavity as the shock wave reflects from the magnetic piston-plasma vacuum interface as a magnetohydrodynamic expansion wave propagating back through the hot plasma. Waves continue to propagate through the plasma as the gas sloshes back and forth in the cavity. The gas comes to rest, filling the entire chamber with a hot plasma containing a uniform azimuthal magnetic field, as shown in figure $2 \mathrm{~d}$. The system is crowbarred by short-circuiting the flat dielectric end walls at the time the reflected shock first reaches the magnetic piston-plasma interface, so that all the magnetic flux, including the driver piston magnetic field, is trapped within the coaxial chamber. At the conclusion of the plasma sloshing, some of this trapped magnetic field has been converted by Ohmic dissipation into plasma thermal energy, and the rest has significantly increased the imbedded insulating magnetic field. Thus as the initially compressed plasma re-expands, it is actualiy. heated further with the final thermodynamic state being at 
a higher temperature than that which existed before the re-expansion. The time for this shoshing to decay is assumed to be several Alfven transit times over the axial length of the chamber.

The quiescent plasma is in contact with and confined by the walls, and thermally insulated by both the azimuthal magnetic field. (which is everywhere parallel to the confining walls) and by a magnetic and thermal boundary layer which develops over the entire surface area where the plasma is in contact with the walls. The interaction between a warm gas and a wall is a classical problem which has been extensively studied. The heat loss from the gas to the wall was first analyzed by Fourier [1-12] who employed a simple constant conduction model. The effects of convection were included later and then the physics of a compressible fluid were added [1-13]. Cold plasma boundary layers have been studied recently for magnetohydrodynamic power generation research [1-14]. Chu's basic study of the behavior of a hot plasma brought into contact with a cold wall [1-15] displayed the formation of a cold dense layer and a corresponding increase in the magnetic field at the wa11, where it was assumed that the thermal conduction of both electrons and ions was in the strong magnetic field limit and where radiative effects were not included. Vekshtein [1-16] recognized that the increased density at the wall could lead to weak field thermal conduction and important radiation losses. Jensen [1-17] extended this work to include line radiation and neutral particles coupled to the plasma by rate equations, and showed the absence of electrical currents in the cold layer immediately adjacent to the wall. Lindemuth $[1-18]$ extended these one-dimensional Cartesian models to study the effects of field line curvature, and demonstrated the formation of convective cells 
characteristic of a wall-induced boundary layer instability in a two-dimensional cylindrical geometry, which enhances thermal conduction losses from the plasma.

The plasma near the wall cools rapidly, by radiation losses and thermal conduction losses, al though this energy loss rate is substantially reduced from that of a hot, unmagnetized, dense plasma brought into contact with a metal wall by employing within the plasma a strong magnetic field (which can be considerably less than that required to magnetically confine the plasma) which is everywhere parallel to the confining wall. The reduced kinetic pressure near the wall drives magnetosonic waves which propagate outward from this thermal boundary layer region, and the resulting convective motion of the plasma, toward the wall, transverse to the magnetic field, induces an electric field, which then generates corresponding currents in the plasma. These currents, while causing local ohmic heating, in turn generate a magnetic field which stiffens the plasma and impedes the motion toward. the wall; the magnetic field thus not only drastically reduces the heat transfer rate to the wall by decreasing the plasma thermal conductivity, but also helps the plasma resist collapsing against the wall.

If the plasma has been heated to above the fusion ignition temperature, the interior volume of the plasma burns and its temperature increases through alpha particle heating. Energetic alpha particles created by thermonuclear reactions deposit their kinetic energy in the electron and ion fluids mainly through Coulomb interactions while orbiting around the azimuthal magnetic field lines, except when a gyro orbit carries an alpha particle into the chamber wall, where its remaining energy is lost and either a cold alpha is left or uther cold 
impurity atoms are sputtered into the plasma boundary layer. Alpha particle heating has not been included in past plasma-wall interaction studies. The general time dependence of the alpha particle heating rate of both electrons and ions has however been modelled previously for other physical situations. Chu [1-19] assumed local and instantaneous alpha particle energy deposition, with the alphas becoming part of the ion Maxwellian and the fraction of energy deposited to electrons and ions given by a numerical approximation of an exact kinetic theory. result [1-20]. Conn' [1-21] examined alpha particle slowing down properties in thermonuclear plasmas using the Fokker-Planck equations, while Sigmar and Joyce [1-22] used the Balescu-Lenard kinetic equation and Rose [1-23] a high-energy approximation in which the alpha particle velocity is taken to always be much larger than the plasma thermal velocities. Kammash [1-24] studied plasma heating by energetic heavy charged particles using binary collision theory, where collective effects are incorporated only through the use of Debye screening in the Coulomb cross section. Willenburg [1-25] modeled the distribution among momentum components for various energy ranges as a function of singleparticle Hamiltonian and canonical momenta (which amounts to specification of the particle's trajectory) in a parallel radially-dependent magnetic field in which orbital motion varied widely because the Larmor. radius was comparable to the plasma dimension, so the excursion distance of the alpha particles out of the plasma had to be taken into account.

The process of heating the plasma interior while cooling its edges continues for a time which depends on both the rate of energy loss and the size of the system. For a certain size and initial state (fill density and fill magnetic field) the fusion energy generated in the hot 
central region is greater than the electromagnetic input energy (in both the magnetic piston and the initial fill magnetic field). This fusion energy is transferred through the chamber walls as neutrons or. heat. The energy deposited in the surrounding thermal blanket is converted to electrical power using a conventional thermal cycle.

Previous analyses $[1-26,1-27]$, done for reactor designs which included a magnetic dam, calculated potential burn fractions and energy gains using simple algebraic arguments based on classical characteristic times. For plasmas shock-heated to high $(T Z 50 \mathrm{keV})$ temperatures, significant burn fractions were found, of ten greater than $50 \%$. Various basic characteristic times (derived in appendix I) for the simplifed reactor concept illustrated in figure 1 are shown in figure 3 , for an intermediate-sized system with a characteristic length $L=50 \mathrm{~cm}$, and for typical values of number density $\left(n \sim 10^{22} / \mathrm{m}^{3}\right)$ and magnetic field ( $B \sim 100$ kGauss). Alpha particle energy deposition to electrons and ions is the fastest process at all temperatures of interest; followed by electron-ion equilibration which is not rapid enough to maintain a single plasma temperature unless most of the alpha particle energy is lost in wall collisions rather than deposited in the plasma. The plasma then cools by bremsstrahlung radiation, before significant thermonuclear burn occurs at lower ( $T \sim 10 \mathrm{keV}$ ) temperatures or after substantial thermonuclear burn occurs at higher $(T \approx 100 \mathrm{keV})$ temperatures. The classical conduction cooling time, based on ion transverse conductivity, is great enough that thermal conduction is not a dominant loss mechanism; the conduction cooling time is the only characteristic time depending on the cavity size, and conduction becomes comparable to radiation only for small $(1.20 \mathrm{~cm})$, low tomperiture 


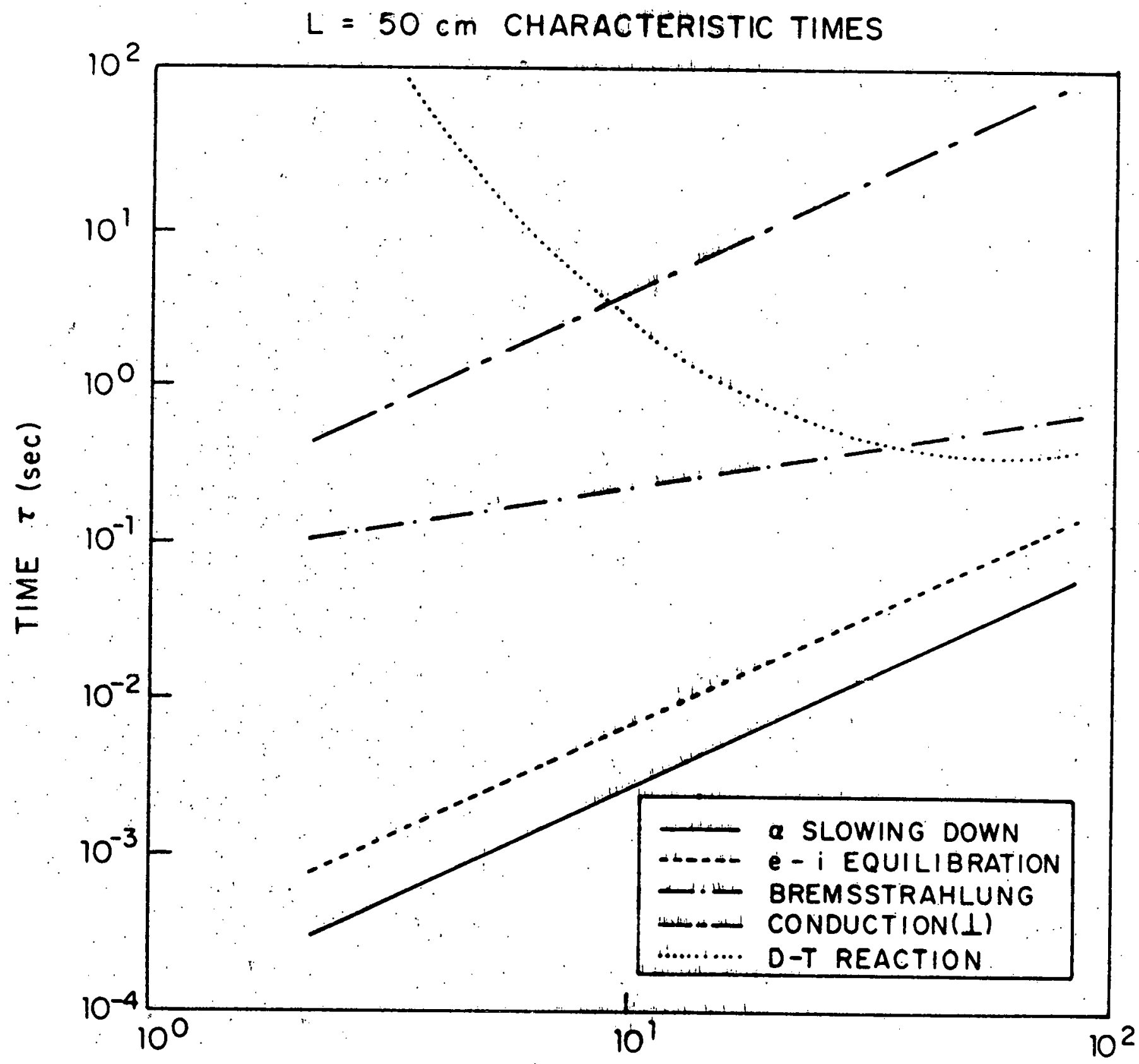

POST-EXPANSION TEMPERATURE $T_{6}(\mathrm{keV})$

Figure 3 (chapter 1) Characteristic times for a plasma with temperature $\mathrm{T}_{6}$, confined between walls $50 \mathrm{~cm}$ apart 
( $\mathrm{T} \leqslant 10 \mathrm{keV}$ ) systems. The burn fraction attainable can be estimated from the characteristic cooling time and reaction time. The deuterium or tritium number density is given by

$$
\frac{d n_{D, T}}{d t}=-\frac{n_{D, T}}{\tau_{R}}
$$

where $\tau_{R}$ is the characteristic DT reaction time. If the plasma is assumed to react for one characteristic bremsstrahlung cooling time $\tau_{b}$, the final number densities are given by

$$
n_{D, T}\left(t=\dot{\tau}_{b}\right)=n_{D, T}(0) \exp \left(-\frac{\tau_{b}}{\tau_{R}}\right)
$$

and the burn fraction is then

$$
f=\frac{n_{\alpha}}{n_{D, T}(0)}=\frac{n_{D, T}(0)-n_{D, T}}{n_{D, T}(0)}=1-\exp \left(-\frac{\tau_{b}^{\prime}}{\tau_{R}}\right)
$$

This burn fraction is independent of number density and magnetic field, and ranges from $8 \%$ at $3 \mathrm{keV}$, through $57 \%$ at $30 \mathrm{keV}$, to $83 \%$ at $100 \mathrm{keV}$; it rises rapidly until $\sim 30 \mathrm{keV}$ and then levels off as it approaches its limiting value of 1 . The thermonuclear energy proportionately rises sharply until $T \sim 30 \mathrm{keV}$, and then slows (while the energy input required to reach the required temperature increases smoothly over the entire range).

A more accurate estimate of the attainable burn fraction and energy gain can be made by studying a one-dimenșional model of the plasma-wall interaction, as is done in this thesis. The bremsstrahlung cooling rate and thermonuclear reaction rate are calculated more: 
precisely from time dependent differential equations, and additional, potentially important, effects such as thermal conduction losses, alpha particle heating (described in appendix II), convection and magnetic diffusion are taken into account. Because the characteristic shock heating time ( microseconds) is short compared to the various plasma times shown in figure 3 , and since the ré-expansion is assumed to occur over only a few Alfven transit times (also $\sim$ microseconds), this onedimensional plasma burn model is applied only to the re-expanded, initially quiescent plasma, and the shock-heating and re-expansion phases are treated as a simple conservation-law algebraic problem which, when solved (in appendix III),yields the energy input required to shock-heat to a given post-expansion plasma temperature and magnetic field.

Such a one-dimensional model by definition does not include several physical effects which may be important and which are inherently twodimensional in nature. Charged particles in an azimuthal radiallydependent magnetic field such as would be found in the cylindrical geometry of figure 1 experience grad $B$ and curvature drifts [1-28] which are of particular importance to the behavior of the high energy alpha particles created throughout the plasma by thermonuclear reactions. These alpha particles drift to the outer wall in times comparable to, but usually shorter than, their characteristic energy deposition time; thus proper modelling of alpha particle heating requires a full twodimensional analysis in cylindrical geometry. While no such study has been done, the problem of a two-dimensional, initial value, timedependent model of a hot, dense, magnetized but "unreacting" plasma brought into sudden contact with a cold wall has been treated recently 
[1-18], and the results show a magnetohydrodynamic wall-induced instability (believed similar to the $Z$-pinch negative radial pressure gradient instability) which enhances thermal conduction losses from the plasma, by a factor of two in the first twenty microseconds in the particular case studied. This instability repetitively grows and stabilizes due to convective stabilization competing with wall thermal losses maintaining unstable profiles, and the conduction enhancement wnuld thus not be expeciled to increase drastically over much longer times. Such two-dimensional loss mechanisms can be approximated in a simple one-dimensional study, but their actual effect remains unknown until a full two-dimensional problem is considered. 
Chapter 2: Physics of a Magnetically Insulated, Thermonuclear Reacting Plasma Contained Between Cold Walls

The one-dimensional problem of a bounded, magnetized, thermonuclear burning plasma contained between two cold walls is studied in this chapter. The magnetohydrodynamic model used includes thermal conduction, resistive diffusion, radiation and alpha particle heating. In the first section, appropriate physics asssumptions are presented and their range of validity is discussed. The magnetohydrodynamic conservation laws and the laws of reaction kinetics are then used to formulate the problem mathematically as a set of partial differential equations, and the appropriate initial and boundary conditions are discussed. The computational scheme used in solving these equations is described in the third section. Finally, the numerical results of the time evolution of the density, temperature and magnetic field profiles are presented in the fourth section.

\section{A. Assumptions and Discussion}

The physical model used is determined by the relationship of the various characteristic lengths and times found in the problem. These are summarized in appendix I, where they are arranged in approximate length and time orderings. In this study, a one-fluid, two-temperature, optically thin plasma is assumed. The only thermonuclear reaction taken into account is the $D(T, n) \alpha$ channel, and the energy carried by the reacting products is only partially absorbed by the plasma itself 
(with all the neutron and part of the alpha particle energy escaping). Classical values of plasma transport coefficients and rate constants are assumed. The plasma has a uniform time-dependent total pressure (kinetic and magnetic). The wall is assumed to be electrically conducting, and at a constant small wall temperature.

The magnetohydrodynamic model, in which the plasma is treated as a condurting fluid, is valid in the regime where the particle mean free path is much smaller than the characteristic distance over which the plasma quantities change appreciably [2-1]. In the presence of a strong magnetic field, this condition is modified and the region of validity of the magnetohydrodynamic approximation is that where the ion gyroradius is much smaller than the particle mean free path [2-2],

$$
\frac{r_{g i}}{\ell_{c}} \ll 1
$$

The ion gyroradius should also be smaller than the dimensions of the plasma, and the ion gyroperiod smaller than the time scales studied, for the continuum approach to be valid [2-3, 2-4].

While the assumption of quasi-neutrality $\left(n_{e}=Z n_{j}\right)$ is violated in a very thin sheath near the wall, whose width is on the order of the Debye length, this thickness is much less than the ion gyroradius (which is itself much smaller than the other characteristic lengths in the problem), and thus charge neutrality holds whenever [2-5]

$$
\frac{\lambda_{D}}{r_{g i}} \ll 1
$$

The Debye lemgth, mesen frece path, and ion gyroradius are piven hy. 
equations (AI.14), (AI.15) and (AI.16), respectively. A comparison of these characteristic lengths for various values of plasma temperature (for a magnetic field of 100 kGauss, and electron and ion number densities of $6.4410^{21} / \mathrm{m}^{3}$ ) is given in the following table:

\begin{tabular}{|c|c|c|c|}
\hline$T(k e V)$ & $\lambda_{D}(m)$ & $r_{g i}(m)$ & $\ell_{c}(m)$ \\
\hline 1 & $2.9 \cdot 10^{-6}$ & $8.210^{-4}$ & 2.4 \\
\hline 3 & $.5 .1 \cdot 10^{-6}$ & $1.410^{-3}$ & $2.010^{1}$ \\
\hline 10 & $9.310^{-6}$ & $2.610^{-3}$ & $2.010^{2}$ \\
\hline 30 & $1.610^{-5}$ & $4.510^{-3}$ & $1.610^{3}$ \\
\hline 100 & $2.910^{-5}$ & $8.210^{-3}$ & $1.7 \cdot 10^{4}$ \\
\hline
\end{tabular}

The ratio in equation (2.1) ranges from $\sim 10^{-4}$ at low temperature to $\sim 10^{-6}$ at high temperature. The ratio in equation (2.2) is $\sim 10^{-3}$, and is constant for a fixed number density and magnetic field.

The electrons and ions attain Maxwellian velocity distributions and have clearly defined temperatures if their respective self-collision times $\tau_{c e}$ and ${ }^{\tau_{c j}}$ are smaller than the characteristic thermonuclear reaction time $\tau_{R}$,

$$
\frac{\tau_{c e}}{\tau_{R}} \ll \frac{\tau_{c i}}{\tau_{R}} \ll 1
$$

Their temperature difference must be taken into account if the electronion equilibration time is comparable to the reaction rate,

$$
\frac{\tau}{\tau_{\mathrm{ej}}} \stackrel{<1}{\tau_{\mathrm{R}}}
$$


The ion and electron self-collision times and the electron-ion equilibration time are given by equations (AI.1), (AI.2) and (AI.3), respectively. A comparison of these characteristic times, and of the reaction time given by equation (AI.9), for various values of plasma temperature is given in the following table:

\begin{tabular}{lllll}
$T(k e V)$ & ${ }_{c e}^{\tau}(s c c)$ & ${ }^{\tau}{ }_{c j}(s e c)$ & ${ }^{\tau}{ }_{e j}(\mathrm{sec})$ & $\tau_{\mathrm{R}}(\mathrm{sec})$ \\
\hline 1 & $1.010^{-7}$ & $7.110^{-6}$ & $2.510^{-4}$ & $4.410^{4}$ \\
3 & $4.910^{-7}$ & $3.310^{-5}$ & $1.210^{-3}$ & $1.610^{2}$ \\
10 & $2.710^{-6}$ & $1.810^{-4}$ & $6.410^{-3}$ & 2.6 \\
30 & $1.310^{-5}$ & $8.810^{-4}$ & $3.110^{-2}$ & 0.45 \\
100 & $7.210^{-5}$ & $4.910^{-3}$ & $1.710^{-1}$ & 0.39
\end{tabular}

The ratios in equation (2.3) are always less than $\sim 10^{-2}$, but at high temperatures the ratio in equation (2.4) ranges from 0.07 to 0.5 . The difference in ion and electron temperatures must also be taken into account when the alpha particle slowing down time is comparable to the electron-ion equilibration time,

$$
\frac{\tau_{\alpha}}{\tau_{\mathrm{ei}}} \sim \frac{\tau_{\mathrm{th}}}{\tau_{\mathrm{ej}}} \sim 1
$$

The alpha particle slowing down time $\tau_{\alpha}$ (the classical alpha-electron equilibration time) and the alpha particle thermalization time ${ }^{\tau}$ th (the time required for the alpha particle to thermatize to the ion fluid background in the presence of both electrons and ions) are given in equations (AI.6) and (AI.7). A comparison of these charac- 
teristic times with the electron-ion equilibration time and the reaction time is given in the following table for different values of plasma temperatures;

\begin{tabular}{lllll}
$T(\mathrm{keV})$ & $\tau_{\alpha}(\mathrm{sec})$ & $\tau_{t h}(\mathrm{sec})$ & $\tau_{\mathrm{ej}}(\mathrm{sec})$ & $\tau_{R}(\mathrm{sec})$ \\
\hline 1 & $1.010^{-4}$ & $4.710^{-4}$ & $2.510^{-4}$ & $4.410^{4}$ \\
3 & $4.810^{-4}$ & $1.710^{-3}$ & $1.210^{-3}$ & $1.610^{2}$ \\
10 & $2.610^{-3}$ & $6.310^{-3}$ & $6.410^{-3}$ & 2.6 \\
30 & $1.210^{-2}$ & $1.710^{-2}$ & $3.110^{-2}$ & 0.45 \\
100 & $6.810^{-2}$ & $3.410^{-2}$ & $1.710^{-1}$ & 0.39
\end{tabular}

The ratio in equation (2.5) varies from $\sim 2$ at low temperatures to $\sim 0.2$ at high temperatures, and thus alpha particle heating could maintain unequal electron and ion temperatures.

The radiation emitted by a plasma will be able to escape from the plasma if its characteristic absorption length is larger than the plasma dimension,

$$
\frac{\lambda}{\ell}>1
$$

The frequency-dependent photon mean free path corresponding to the sharply peaked maximum in the power emitted due to ion-electron bremsstrahlung is given by equation (AI.17), and varies from $\sim 10^{15} \mathrm{~m}$ at low temperatures to $\sim 10^{22} \mathrm{~m}$ at high temperatures (for a number density of $\sim 10^{22} / \mathrm{m}^{3}$ ).

Similarly, the neutrons (which can be thermalized only by 
nuclear force interactions with the plasma ions) will be able to escape from the plasma if their absorption mean free path is much greater than the plasma dimension,

$$
\frac{l_{n}}{\ell} \gg 1
$$

The neutron mean free path is given by equation (AI.18), and is on the urder of $10^{6} \mathrm{~m}$ for an ion number density of $10^{22} / \mathrm{m}^{3}$.

The ratios in equations (2.6) and (2.7) are therefore easily satisfied for plasma dimensions of $0.1 \mathrm{~m}$ to $1 \mathrm{~m}$, for various plasma temperatures and for number densities $\sim 10^{22} / \mathrm{m}^{3}$.

The alpha particles can be contained in the magnetized plasma when the alpha particle gyroradius is smaller than the plasma dimension,

$$
\frac{r_{\alpha}}{\ell}<1
$$

The gyroradius is given by equation (AI.19), and for a typical magnetic field strength of 100 kGauss the ratio in equation (2.8) ranges from $\sim 0.3$ to $\sim 0.05$ as the plasma dimension varies from 0.2 to 1 meter. Thus the alpha particle energy is only partially absorbed and the alpha particle confinement must be modelled.

While there are seven possible nuclear reaction channels in a $D-T$ plasma [2-6], only the four reactions $D(T, n) \mathrm{He}^{4}, D(D, p) T, D(D, n) \mathrm{He}^{3}$ and $\mathrm{He}^{3}(D, p) \mathrm{He}^{4}$ are generally considered in thermonuclear problems. The reaction probabilities for these four reactions are shown in figure 1. The value of $\langle\sigma v\rangle$ is several times greater for the $D-T$ reaction than for the $D-D$ and $\mathrm{He}^{3}-\mathrm{D}$ reactions, for the ion temperatures of interest. 


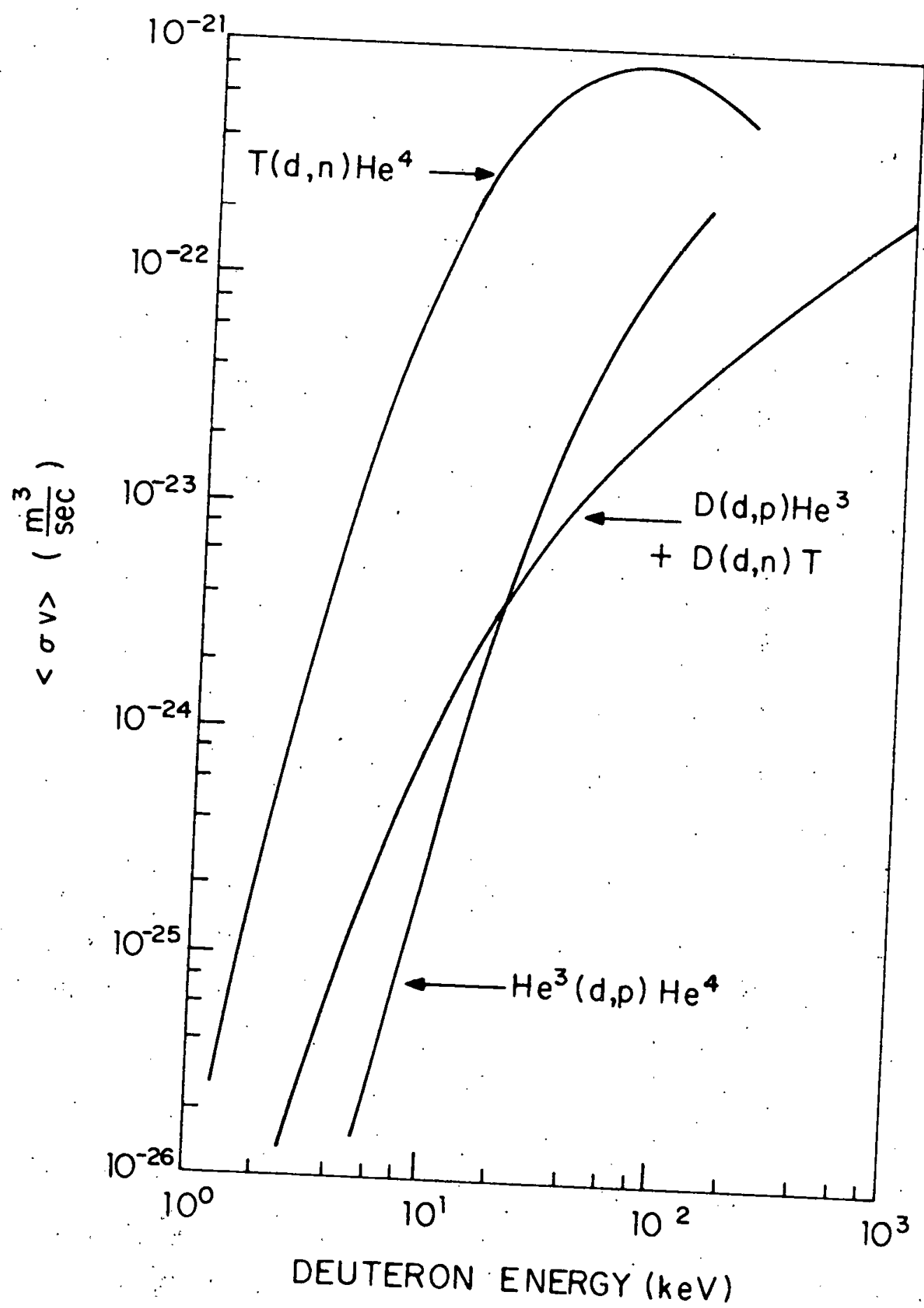

Figure 1 (chapter 2) Reaction probabilities for several thermonuclear reactions in a deuterium-tritium plasma 
The $D-T$ reaction is also favored from concentration arguments, since the $\mathrm{He}^{3}-\mathrm{D}$ reaction is lower in the chain and the two $D-D$ branches involve two deuterons. Thus only the D-T reaction is assumed to occur.

The plasma is a nonequilibrium system, evinced by the temperature difference between the electrons and ions and the radiation optical thinness of the plasma at each point, and by the tempcrature, density, velocity and magnetic field gradients between different points. The plasma tries to attain equilibrium by establishing fluxes of energy, momentum and mass, and through diffusion of the magnetic field, between regions with different temperatures, densities, velocities and magnetic fields, and by radiating continuously and transferring energy between the electrons and ions. The values of the rate constants and transport properties governing these processes are assumed to have their classical values; no instabilities or turbulence are assumed.

The buildup of the temperature and magnetic field gradients. near the wall drive magnetosonic waves into the hot plasma interior. The magnetoacoustic speed is given by

$$
\therefore m=\frac{\gamma k T T}{m_{i}}+\frac{B^{2}}{\mu_{0} \rho}
$$

and is a characteristic velocity of the problem. For a magnetic field of 100 kGauss, the magnetosonic speed varies from $210^{6} \mathrm{~m} / \mathrm{sec}$ to $310^{6} \mathrm{~m} / \mathrm{sec}$ for plasma temperatures between 1 and $100 \mathrm{keV}$. The time for the magnetoacoustic waves to cross the system is on the order of $10^{-7} \mathrm{sec}$, much shorter than any other characteristic time, except the electron self-collision time. The total plasma pressure (kinetic and 
magnetic) can therefore be assumed uniform in the plasma, al though varying with time.

The wall is assumed to be at a constant temperature, which is valid if the wall thermal conductivity is very large (so that the wall can conduct the heat at a rate comparable to the rate at which the plasma transfers heat to the wal1). A large thermal conductivity implies a large electrical conductivity [2-7], which in turn implies small ohmic losses in the wall. If the wall is assumed to be a metal such as copper, the total energy it can gain before melting is approximately

$$
e=\rho c_{V}\left(T_{M}-T_{0}\right)=\frac{k}{k}\left(T_{M}-T_{0}\right) \sim 3.510^{9} \frac{\mathrm{J}}{\mathrm{m}^{3}}
$$

(The material data used for copper are a thermal conductivity $\kappa=394 \frac{\text { watts }}{\mathrm{m}^{\circ} \mathrm{c}}$, a thermal diffusivity $k=1.1410^{-4} \frac{\mathrm{m}^{2}}{\mathrm{sec}}$ and $a$ melting temperature $T_{M}=1346^{\circ} \mathrm{K}[2-8]$ with a room temperature $T_{0}=$ $300^{\circ} \mathrm{K}$ ) A simple thermal conduction model for a plasma boundary layer gives as the total energy loss from the plasma [2-9]

$$
\begin{aligned}
\mathrm{q}\left(\frac{\mathrm{J}}{\mathrm{cm}^{2}}\right) & =6.310^{-24} \frac{\left[T\left({ }^{\circ} \mathrm{K}\right)\right]^{3 / 4}\left[n_{j}\left(\mathrm{~cm}^{-3}\right)\right]^{3 / 2}(\mathrm{ln} \Lambda)^{1 / 2} t^{1 / 2}}{B(\text { Gauss })} \\
& \simeq 1.610^{2} \sqrt{t}
\end{aligned}
$$

for a plasma temperature of $10 \mathrm{keV}$, magnetic field of 100 kGauss and number density of $6.4410^{21} / \mathrm{m}^{3}$. The energy loss above equals the energy gain a $1 \mathrm{~cm}$ thick copper wall can tolerate before bulk melting at $t$ $500 \mathrm{sec}$, and conversely the mean wall temperature rise for $t \leadsto 1 \mathrm{sec}$ is 
$\sim 46^{\circ} \mathrm{K}$. The wall temperature, even when it rises, is always much smaller than the plasma temperatures, and the plasma effectively sees a constant small wall temperature.

B. Equations and Boundary Conditions

In this section, the assumptions made ill the last section are used together with the conservation and reaction kinetics laws to formulate a set of differential equations that govern the plasma behavior, and the appropriate initial and boundary conditions discussed. Continuity of number density for each particle species $(s=D, T$, $n, \alpha$, and e) gives

$$
\frac{\partial n_{s}}{\partial t}+\frac{\partial}{\partial x}\left(n_{s} u\right)=s_{s}
$$

where $S_{S}$ is the species source function, giving the net rate of increase of the individual specie number densities. . For the D-T reaction these source functions are

$$
\begin{gathered}
S_{\alpha}=S_{n}=-S_{D}=-s_{T}=n_{D} n_{T}<\sigma V> \\
S_{e}=0
\end{gathered}
$$

Since the plasma is assumed to remain electrically. neutral at a11 times, the ion and electron components undergo the same dynamic motion, characterized by the single-fluid bulk velocity $u$. The plasma equation of motion in conservation form is 


$$
\frac{\partial}{\partial t}(\rho u)+\frac{\partial}{\partial x}\left(\rho \frac{u^{2}}{2}+p+\frac{B^{2}}{2 \mu_{0}}\right)=-P_{n}
$$

where the mass density $\rho$ is given by

$$
\rho=m_{D} n_{D}+m_{T} n_{T}+m_{\alpha} n_{\alpha}+m_{e} n_{e}
$$

and the pressure $p$ is the sum of the partial pressure

$$
p=p_{D}+p_{T}+p_{\alpha}+p_{e}
$$

There is no contribution to the plasma density from the neutrons, since they are assumed to escape from the plasma immediately, carrying an amount of momentum $P_{n}$ with them. (Conversely, even when the alpha particles are not confined and lose all their energy in wall collisions, all the alpha particles are assumed to remain as part of the plasma.) When combined with the number density expressions given by equations (2.10) and (2.11), the plasma equation of motion becomes

$$
-\dot{P}_{n}=\rho \frac{\partial u}{\partial t}+\rho u \frac{\partial u}{\partial x}+\frac{\partial}{\partial x}\left(p+\frac{B^{2}}{2 \mu_{0}}\right)+u\left(-m_{D} n_{D} S_{D}-m_{T} n_{T} S_{T}+m_{\alpha} n_{\alpha} S_{\alpha}\right)
$$

If the change in momentum of an individual species $s$ is defined as

$$
\dot{p}_{s}=m_{s} n_{s} s_{s} u
$$

and if the approximation $m_{n} \sim m_{D} \sim m_{T} \sim m_{\alpha}$ is used, the plasma equation of motion takes on the standard form. 


$$
\rho\left(\frac{\partial u}{\partial t}+u \frac{\partial u}{\partial x}\right)=-\frac{\partial}{\partial \dot{x}}\left(p+\frac{B^{2}}{2 \mu_{0}}\right)
$$

The Lorentz force is represented by the $\frac{\mathrm{B}^{2}}{2 \mu_{0}}$ term; with the current eliminated in favor of the magnetic field using

$$
f_{x}=j_{y} B_{z}=\left(-\frac{1}{\mu_{0}} \frac{\partial B_{z}}{\partial x}\right)_{z}=-\frac{\partial}{\partial x}\left(\frac{B_{z}^{2}}{2 \mu_{n}}\right)
$$

When the magnetoacoustic speed given by equation (2.9), which is the characteristic velocity of the system of equations given in this section, is large enough that it can be considered infinite, equation (2.16) can be replaced by the pressure balance equation

$$
p+\frac{B^{2}}{2 \mu_{0}}=p_{\text {wail }}
$$

The plasma is, of course, in pressure balance to start with, but as the ions react they change the plasma density and energy, and as the magnetic field diffuses it will alter the field distribution, resulting in forces that drive the plasma into motion. This motion will be small in comparison with other processes and can adequately be followed by maintaining a static pressure balance. Maxwell's equations of electrodynamics give Faraday's law in conservation form as

$$
\frac{\partial B}{\partial t}+\frac{\partial}{\partial x}(u B)-\frac{1}{\mu_{0}} \frac{\partial}{\partial x}\left(\eta \frac{\partial B}{\partial x}\right)=0
$$

where $n$ is the classical electrical resistivity transverse to the magnetic field, given by [2-10] 


$$
\begin{aligned}
n_{\perp}=\frac{n_{L}}{\gamma_{E B}} & =\left(\frac{32}{3 \pi}\right) \frac{\pi^{3 / 2} m_{e}{ }^{1 / 2} Z_{e}{ }^{2} c^{2} \ell n \Lambda}{2(2 k T)^{3 / 2}} \\
& =1.26910^{-19 \frac{l n \Lambda}{T^{3 / 2}}}
\end{aligned}
$$

where $[\eta]=\sec ,[T]=k e V$ and $\ln \Lambda$ is the Coulomb logarithm, defined in appendix I.

The electrons and ions, having two different temperatures, satisfy separate energy equations, given in conservation form by

$$
\frac{\partial}{\partial t}\left(\frac{3}{2} n_{i, e^{k T}} ;, e\right)+\frac{\partial}{\partial x}\left(\frac{3}{2} n_{i, e^{k T}}, e^{u}\right)=-p_{i, e} \frac{\partial u}{\partial x}+Q_{i, e}
$$

(The ion fluid is assumed to consist of deuterons, tritons and thermalized alpha particles, all with an average mass of $2.5 \mathrm{amu}$, as discussed in appendix II.) This equation can be combined with the electron number density equation to give

$$
\frac{3}{2} n_{e} k\left(\frac{\partial T_{e}}{\partial t}+u \frac{\partial T_{e}}{\partial x}\right)=-p_{e} \frac{\partial u}{\partial x}+Q_{e}
$$

where $Q_{e}$ is the power input to the electrons per unit volume, due to various processes such as thermal conduction, electron-ion equilibration, radiation, Joule heating and alpha particle heating. The thermal conduction term is given by.

$$
Q_{c, e}=\frac{\partial}{\partial x}\left(\kappa_{e} \frac{\partial T_{e}}{\partial x}\right)
$$

where $k$ is the classical transverse thermal conductivity, given by 
[2-10]

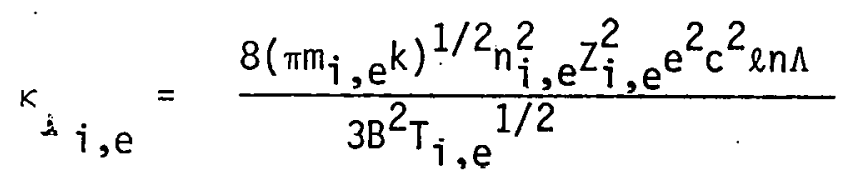

The electron thermal conductivity in equation (2.23) is thus given by

$$
\kappa_{e}=7.3010^{-23} \frac{n_{e} \ell n \Lambda}{B^{2 /} T_{e}{ }^{1 / 2}}
$$

(In this section, unless noted otherwise, the units used are $[n]=m^{-3}$, $[T]=k e V$, and $[B]=$ kGauss.) The electron-ion equilibration term is given by

$$
Q_{x, e}=\frac{3}{2} n_{e} e^{\frac{T_{j}-T_{e}}{\tau_{e i}}}
$$

where the classical electron-ion equilibration time $\tau_{e i}$ is given by equation (AI.3).

Because of the presence of low plasma temperatures near the wall due to conduction losses, and because of the buildup of alpha particles near the wall due to collisions, the radiation term includes three major processes -- bremsstrahlung, line or excitation radiation and recombination. While bremsstrahlung dominates throughout much of the problem, line radiation becomes of significance when $T \lesssim 25 \mathrm{eV}$, while recombination is important for $\mathrm{T} \lesssim 100 \mathrm{eV}$. The specific radiation power emitted by the electrons is given by [2-11]

$$
P_{\text {rad }}=n_{e}\left[1.6 \times 10^{-27}\left(\sum_{z} z^{2} n_{z}\right) T^{1 / 2}\right.
$$




$$
\left.+610^{-22}\left(\sum_{z} z^{4} n_{z}\right) T_{e}^{-1 / 2}+810^{-17}\left(\sum_{z} z^{6} n_{z}\right) T_{e}^{-3 / 2}\right]
$$

where $T_{e}$ is the electron temperature in ${ }^{\circ} \mathrm{K}, \mathrm{n}_{\mathrm{e}}$ and $\mathrm{n}_{z}$ are the electron and ion-of-atomic-number- $Z$ number density in $\mathrm{cm}^{-3}$, and $P_{\text {rad }}$ is the radiated power per cc of plasma in erg/sec. The three terms are bremsstrahlung, recombination, and line radiation, in that order. For a plasma with equal numbers of deuterons and tritons, this expression becomes, in the usual units,

$$
\begin{aligned}
& Q_{\text {rad }}=n_{e}\left[6.69 \cdot 10^{-21}\left(n_{D}+2 n_{\alpha}\right) T_{e}^{1 / 2}\right. \\
& \left.+2.210^{-22}\left(n_{D}+8 n_{\alpha}\right) T_{e}^{-1 / 2}+2.5410^{-24}\left(n_{D}+32 n_{\alpha}\right) T_{e}^{-3 / 2}\right]
\end{aligned}
$$

The Joule heating term is given by

$$
Q_{j}=n j^{2}=\frac{\eta}{\mu_{0}^{2}}\left(\frac{\partial B}{\partial x}\right)^{2}
$$

and the alpha particle heating term $Q_{\alpha e}$ is given by equation (AII.19). (The spacial and time-dependent modelling of the alpha particle energy deposition is described in detail in appendix II.) Combining equations (2.22) through (2.29) gives as the electron temperature equation 


$$
\begin{aligned}
\frac{3}{2} n_{e} k\left(\frac{\partial T_{e}}{\partial t}+u \frac{\partial T_{e}}{\partial x}\right)=-p_{e} \frac{\partial u}{\partial x}+\frac{\partial}{\partial x}\left(k_{e} \frac{\partial T_{e}}{\partial x}\right)+\frac{3}{2} n_{e} \cdot \frac{T_{j}-T_{e}}{T_{e j}} \\
+\frac{\eta}{\mu_{o}^{2}}\left(\frac{\partial B}{\partial x}\right)^{2}+Q_{\alpha e}-C_{f f} n_{e} \cdot\left(\sum z^{2} n_{z}\right) T_{e}{ }^{1 / 2}-C_{f b} n_{e}\left(\sum z^{4} n\right) T_{e} e^{-1 / 2} \\
-C_{b b} n_{e}\left(\sum z^{6} n_{z}\right) T_{e}{ }^{-3 / 2}
\end{aligned}
$$

where $C_{f f}, C_{f b}$ and $C_{b b}$ are numerical coefficients for bremsstrahlung, recombination and line radiation given by equation (2.27).

When the ion energy equation given by equation (2.21) is combined with the number density expressions given by equation (2.10), it becomes

$$
\frac{3}{2} n_{i} k\left(\frac{\partial T_{i}}{\partial t}+u \frac{\partial T_{i}}{\partial x}\right)+\frac{3}{2} k T_{i}\left(-S_{D}-S_{T}+S_{\alpha}\right)=-p_{i} \frac{\partial u}{\partial x}+Q_{i}
$$

where $Q_{j}$ is the power input to the ions per unit volume due to thermal conduction, electron-ion equilibration, alpha particle heating and neutron loss. If each heavy particle is assumed to carry an energy of $\frac{3}{2} k T_{i}$, so that the change in specific energy of any individual species is

$$
Q_{S}=\frac{3}{2} k T_{i} S_{S}
$$

and so that the thermal energy carried away by neutrons escaping the system is $Q_{n}=-\frac{3}{2} k T_{i} S_{n}$, the source function terms cancel:

The thermal conduction term is given by

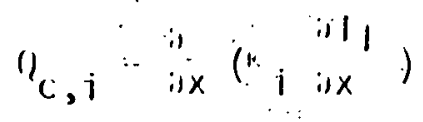


where the classical ion transverse thermal conductivity is given by equation (2.24) as

$$
\kappa_{i}=4.98610^{-21} \frac{n_{i} \ln \Lambda}{B^{2} T_{i}^{1 / 2}}
$$

The electron-ion equilibration term is given by

$$
Q_{x i}=\frac{3}{2} n_{i} k \frac{T_{e}-T_{i}}{\tau_{e i}}
$$

which by energy conservation is $-Q_{x e}$ of equation (2.26), and the alpha heating term $Q_{\alpha j}$ is given by equation (AII.19). The ion temperature equation is therefore

$$
\frac{3}{2} n_{i} k\left(\frac{\partial T_{i}}{\partial t}+u \frac{\partial T_{i}}{\partial x}\right)=-p_{i} \frac{\partial u}{\partial x}+\frac{\partial}{\partial x}\left(k_{i} \frac{\partial T_{i}}{\partial x}\right)+\frac{3}{2} n_{i} k \frac{T_{e}-T_{i}}{\tau_{e i}}+Q_{\alpha_{j}}
$$

where the ion pressure is given by

$$
\dot{p}_{i}=\left(n_{D}+n_{T}+n_{\alpha}\right) k T_{i}
$$

Equations (2.10), (2.16) or (2.18), (2.19), (2.30) and (2.35) form a set of differential equations for the plasma number densities, velocity, pressure, magnetic field and temperatures, which can be used to study the behavior of an initially uniform, fully ionized plasma contained between two cold walls. The coordinate system is shown in figure 2; the problem is symmetric about $x=\ell=\frac{L}{2}$ (the walls are assumed located at $x=0$ and $x=L$ ). The plasma is considered infinite in both the 


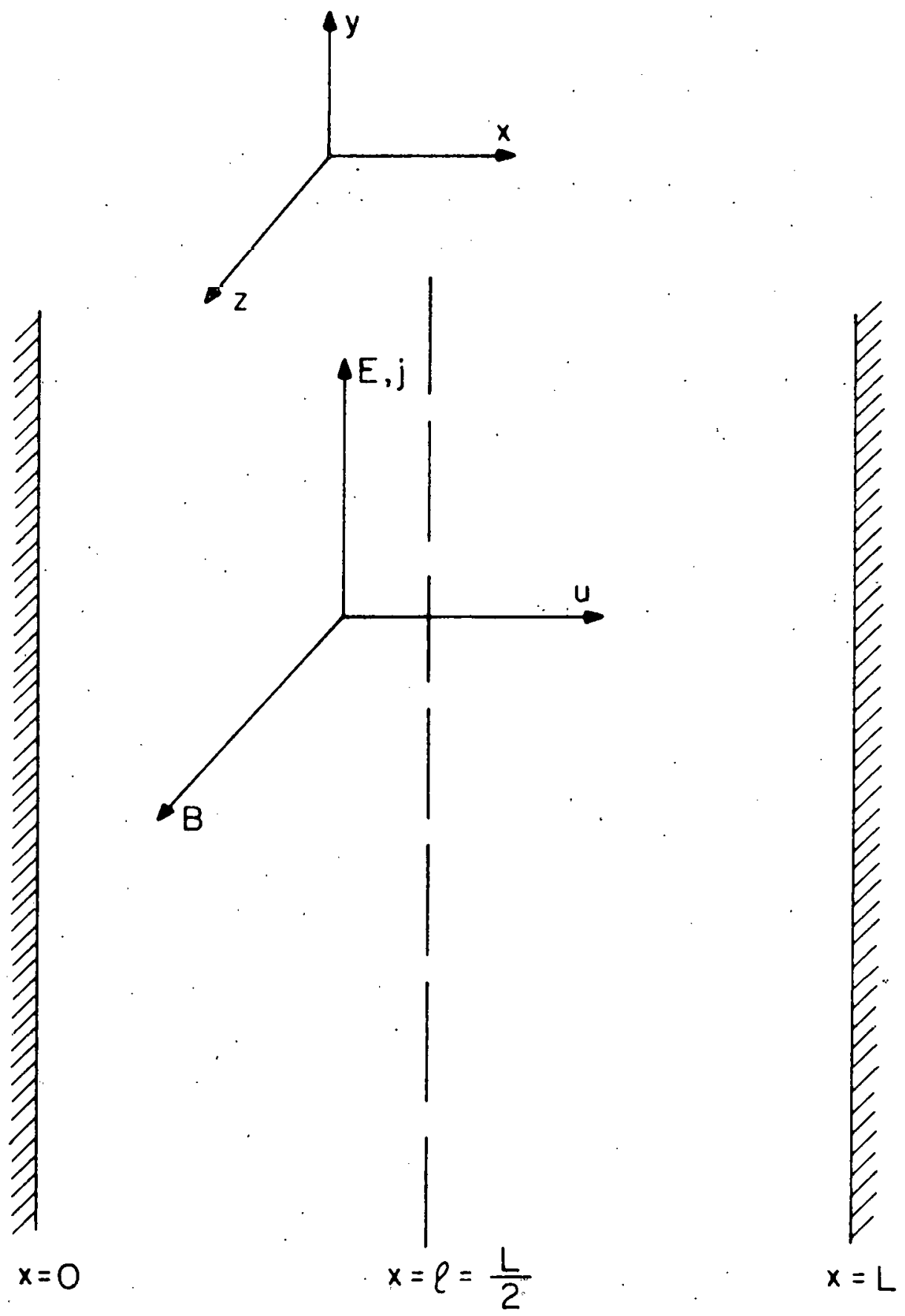

Figure 2 (chapter 2) Coordinate system for a one-dimensional plasmawall study 
$y$ and $z$ directions. Initially the plasma is uniform and stationary, so that

$$
\begin{aligned}
& T_{j}(x, 0)=T_{e}(x, 0)=T_{0} \\
& 2 n_{D}(x, 0)=2 n_{T}(x, 0)=n_{e}(x, 0)=n_{0} \\
& n_{\alpha}(x, 0)=n_{n}(x, 0)=0 \\
& B(x, 0)=B_{0} \\
& u(x, 0)=0
\end{aligned}
$$

The plasma at the wall is assumed to have a constant temperature, giving

$$
T_{i}(0, t)=T_{e}(0, t)=T_{w}
$$

and, since the particle flux through the wall is zero,

$$
u(0, t)=0
$$

At the symmetry plane the boundary conditions are

$$
\begin{gathered}
\frac{\partial T_{i}}{\partial x}(l, t)=\frac{\partial T_{e}}{\partial x}(l, t)=0 \\
\frac{\partial n_{e}}{\partial x}(l, t)=\frac{\partial n_{D, T}}{\partial x}(l, t)=\frac{\partial n_{\alpha}}{\partial x}(l, t)=0
\end{gathered}
$$




$$
\begin{aligned}
\frac{\partial B}{\partial x}(l, t) & =0 \\
u(l, t) & =0
\end{aligned}
$$

C. Numerical Method of Solution

The equations derived in the previous section are rewritten for convenience in Lagrangian form and the difference equations used to solve the system numerically are described, together with the zoning and boundary conditions appropriate for a plasma-wall interface.

The motion of the plasma is followed by using a Lagrangian reference frame in which the position $x$ of a given plasma element is expressed in terms of its original position $x_{0}$ and time $t$. Instead of $x_{0}$ it is convenient to introduce the transformed Lagrangian variable $\xi$ defined by

$$
\xi=\frac{1}{\rho_{0}} \int_{0}^{x} \rho\left(x^{\prime}, t\right) d x^{\prime}
$$

so that the Eulerian position $x$ of a given fluid element is given by $x=x(\xi, t) \quad\left(\rho_{0}\right.$ is the reference density). The relative volume $V$ is defined by

$$
V=\frac{\delta x}{\delta \xi}=\frac{\rho}{\rho}
$$

and the Eulerian derivatives are given by 


$$
\begin{aligned}
& \left.\left.\left.\left.\frac{\partial}{\partial t}\right)_{X} \rightarrow \frac{\partial}{\partial t}\right)_{\xi}-\frac{u}{V} \frac{\partial}{\partial \xi}\right)_{t}=\frac{d}{d t}-\frac{u}{V} \frac{\partial}{\partial \xi}\right)_{t} \\
& \left.\left.\frac{\partial}{\partial x}\right)_{t} \rightarrow \frac{1}{V} \frac{\partial}{\partial \xi}\right)_{t}
\end{aligned}
$$

The Lagrangian number density $\bar{n}_{S}(\xi, t)$ of a plasma component species (such that $\rho_{0} \equiv \sum_{s} m_{s} \bar{n}_{s}$ ) is the density defined in terms of the original configuration and remains the same in time except for addition and depletion of particles resulting from reactions described by the species source functions given in equation (2.11). The actual number densities are given by

$$
n_{s}(\xi, t)=\frac{\bar{n}_{s}(\xi, t)}{V(\xi, t)}
$$

The dependent. variables are the number densities $\left(n_{e}, n_{D}=n_{T}\right.$ and $\left.n_{\alpha}=n_{n}\right)$, the magnetic field $(B)$, the electron and ion temperatures $\left(T_{e}\right.$ and $\left.T_{j}\right)$, and the relative volume $(V)$. The number density equations become

$$
\frac{d n_{\dot{s}}}{d t}=s_{s}-\frac{n_{s}}{V} \frac{d V}{d t}
$$

using the relationship $\frac{\partial u}{\partial x} \approx \frac{1}{V} \frac{d V}{d t}$. Using this expression the magnetic field equation becomes

$$
\frac{d B}{d t}=-\frac{B}{V} \frac{d V}{d t}-\frac{1}{\mu_{0} V} \frac{\partial}{\partial \xi}\left(\frac{n}{v} \frac{\partial B}{\partial \xi}\right)
$$

The electron and ion temperature equations (2.30) and (2.35) respectively 
transform to

$$
\begin{aligned}
\frac{d T_{e}}{d t}= & -\frac{2}{3} \frac{T_{e}}{V} \frac{d V}{d t}+\frac{2}{3 n_{e} V} \frac{\partial}{\partial \xi}\left(\frac{r_{e}}{V} \frac{\partial T_{e}}{\partial \xi}\right)+\frac{T_{i}-T_{e}}{\tau_{e i}} \\
& +\frac{2}{3 n_{e}} \frac{n_{\mu}}{2 V^{2}}\left(\frac{\partial B}{\partial \xi}\right)^{2}+Q_{\alpha e}-\frac{2}{3} C_{f f}\left(\sum_{z} z^{2} n_{z}\right) T_{e}^{1 / 2} \\
& -\frac{2}{3} C_{b f}\left(\sum_{z} z^{4} n_{z}\right) T_{e}^{-1 / 2}-\frac{2}{3} C_{b b}\left(\sum_{z} z^{6} n_{z}\right) T_{e}^{-3 / 2} \\
\frac{d T_{i}}{d t}= & -\frac{2}{3} \frac{T_{i}}{V} \frac{d V}{d t}+\frac{2}{3 n_{j} V} \frac{\partial}{\partial \xi}\left(\frac{\kappa i}{V} \frac{\partial T_{j}}{\partial \xi}\right)+\frac{T_{e}-T_{i}}{\tau_{e j}}+Q_{\alpha j}
\end{aligned}
$$

The system of differential equations (2.51) through (2.54) is replaced by a set of finite difference equations which are used to advance the plasma variables in time, with the time step number given by the variable superscript. The finite intervals, or zones, into which the spatial region of interest is divided, and which determine the points in space at which the dependent variables are defined, are shown in figure 3 [2-12]. The region of interest is divided into $J=20$ cells, or zones, and all dependent variables are defined at $J+2$ points. The cell-centered values such that $1 \leq j \leq J$ are interpreted physically as being "average" values, so that, for example, the total mass in the problem is equal to the sum of the products of the density and cell volumes. The value of the dependent variables at $j=0$ and $j=J+1$ (corresponding to the walls located at $x=0$ and $x=L)$ are interpreted as boundary values; they are considered to be located one half cell away from the values at $j=1$ and $j=J$, respectively, and they are not included in the determination of integral quantities such as mass or thermal energy. In the difference equations 


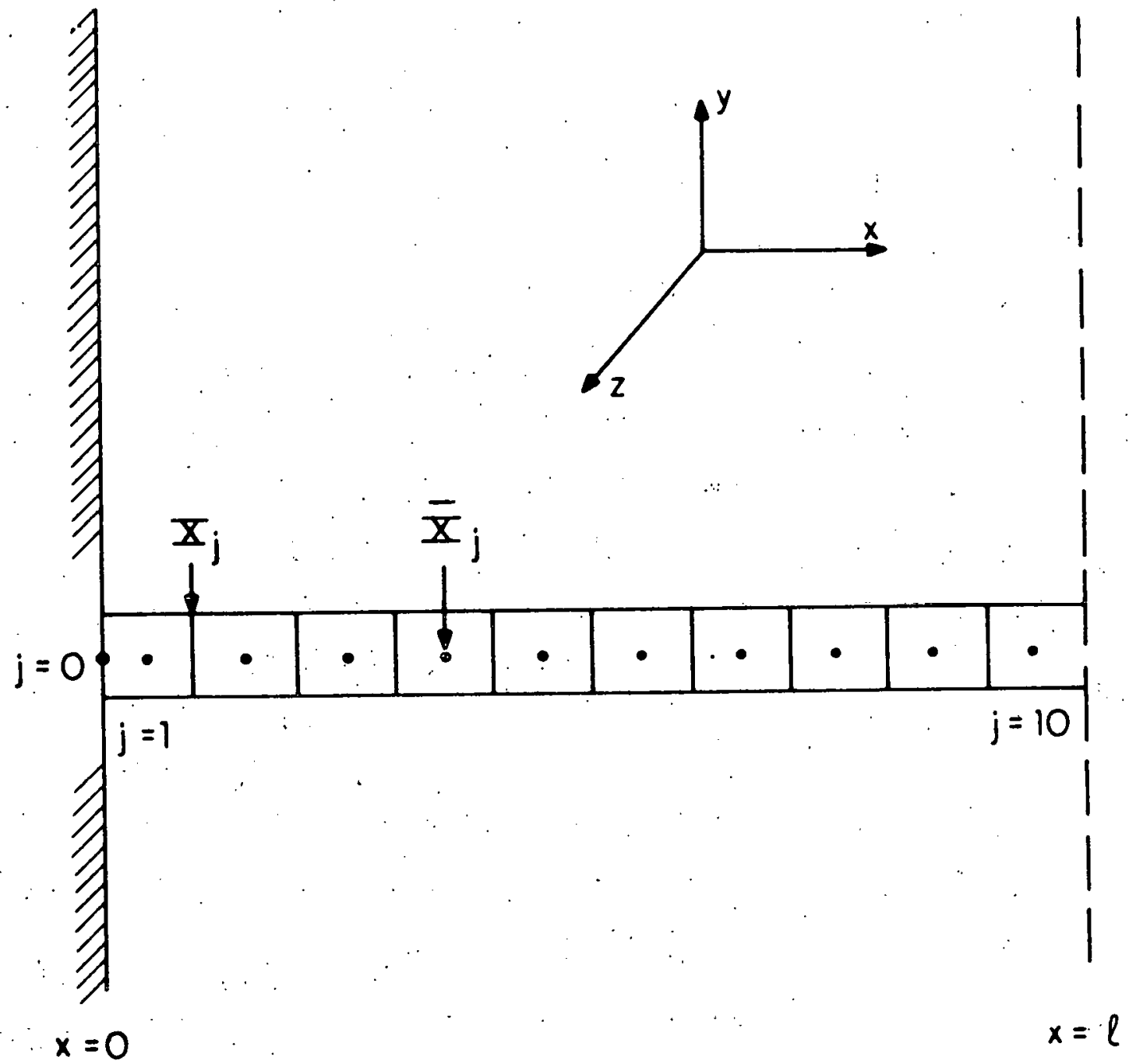

Figure 3 (chapter 2) Spacial zoning for the geometry. shown in figure 2 
described below, integer subscripts refer to cell-centered values with $1 \leq j \leq J$, while half-integer subscripts refer to average interface values given by

$$
g_{j+\frac{1}{2}}=\frac{1}{2}\left(g_{j+1}+g_{j}\right) \quad 1 \leq j \leq j
$$

The boundary conditions at $j=0$ will be discussed later in this section, and are analogous to those at $j=j+1$.

In a given time increment $\Delta t$, the volume of each cell is changed by a factor y required to restore pressure balance; the plasma conditions are then altered by the ion reaction, the diffusion of magnetic field and the transfer of heat all performed with the cell volumes held fixed. At the end of this step each cell has new values of number density, temperature and magnetic field, denoted by asterisks, namely $\bar{n}_{i}^{*}, e, T_{i}^{*}, e$ and $B^{*}$ (not adjusted for pressure balance). The volume of each cell is again changed by a factor $y$ at the beginning of the next time step, and the whole procedure is repeated to continue advancing in time.

The factor $y$ is given by [2-13]

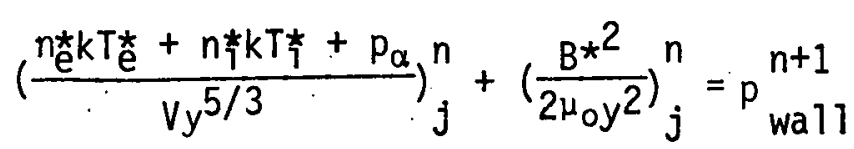

and can be solved for at each cell by Newton-Raphson iteration. The new cell boundaries are given by advancing across the spatial cell grid using 


$$
\begin{aligned}
& x_{0} \equiv 0 \\
& x_{j}=x_{j-1}+y_{j} v_{j} \Delta \xi
\end{aligned}
$$

where $x_{0}$ corresponds to the wall at $X=\xi=0$, and $x_{j}$ is the right boundary of cell $j$ and the left boundary of cell $j+1$, as shown in figure 3. The position of the outermost meshpoint $x_{J}$ will of course deviate from its correct position $X=\xi=L$ at the other wall. The wall pressure is then slightly adjusted (increased if $X_{J}>L$ and decreased if $\left.X_{J}<L\right)$, and the entire procedure iterated. A few Newton-Raphson iterations calculating $p_{\text {wall }}$ are enough to fix the two boundary points to better than $10^{-7} \mathrm{~m}[2-14]$. The resulting cell compression $y$ then gives the pressure balance adjusted plasma quantities as $[2-13]$

$$
\begin{aligned}
& v_{j}^{n+1}=y_{j} v_{j}^{n} \\
& \tilde{B}_{j}^{n+1}=\frac{B_{j}^{*_{j}^{n}}}{y_{j}} \\
& \left(\tilde{T}_{i, e}\right)_{j}^{n+1}=\frac{\left(T_{i, e}^{*}\right)_{j}^{n}}{y_{j}^{2 / 3}} \\
& \left(\tilde{\bar{n}}_{i, e}\right)_{j}^{n+1}=\left(\bar{n}_{i}^{*}, e^{n}\right)_{j}^{n}
\end{aligned}
$$

The tilde $(\sim)$ indicates quantities that have been adjusted for pressure balance.

Next, the effects of the electron and ion thermal conduction and 
mecharical work terms, and the effect of the electron Ohmic heating term, are accumulated implicitly by solving the difference equation

$$
-A_{j}\left(T_{i, e}^{\prime}\right)_{j+1}^{n+1}+B_{j}\left(T_{i, e}^{\prime}\right)^{n+1}-C_{j}\left(T_{i, e}^{\prime}\right)_{j-1}^{n+1}=D_{j}
$$

where the prime (') refers to the pressure-balanced electron and ion temperatures modified by conduction, pV mechanical work and Joule heating. The coefficients in equation (2.61) are given by

$$
\begin{aligned}
& A_{j}=\frac{4 \theta}{3\left(\tilde{n}_{i, e}\right)_{j}^{n+1}} \frac{\Delta t}{\left(\xi_{j+1}-\xi_{j-1}\right)\left(\xi_{j+1}-\xi_{j}\right)} \frac{\left(\kappa_{i, e}\right)_{j+1 / 2}}{v_{j}^{n+1} v_{j+1 / 2}^{n+1}} \\
& C_{j}=\frac{4 \theta}{3\left(\tilde{n}_{i, e}\right)_{j}^{n+1}} \frac{\Delta t}{\left(\xi_{j+1}-\xi_{j-1}\right)\left(\xi_{j}-\xi_{j-1}\right)} \frac{\left(\kappa_{i, e}\right)_{j-1 / 2}}{v_{j}^{n+1} v_{j-1 / 2}^{n+1}} \\
& B_{j}=1+A_{j}+C_{j}+\frac{2}{3} \frac{v_{j}^{n+1}-v_{j}^{n}}{v_{j}^{n+1}} \\
& D_{j}=\left(\tilde{T}_{i, e}\right)_{j}^{n+1}\left[1-a_{j}-c_{j}-\frac{2(1-\theta)}{3} \frac{v_{j}^{n+1}-v_{j}^{n}}{v_{j}^{n}}\right]+a_{j}\left(\tilde{T}_{i, e}\right)_{j+1}^{n+1} \\
& \vdots
\end{aligned}
$$

where

$$
\begin{aligned}
& a_{j}=\frac{4(1-\theta)}{3\left(\tilde{n}_{i, e}\right)_{j}^{n+1}} \frac{\Delta t}{\left(\xi_{j+1}-\xi_{j-1}\right)\left(\xi_{j+1}-\xi_{j}\right)} \frac{\left(\kappa_{i, e}\right)_{j+1 / 2}}{v_{j}^{n} v_{j+1 / 2}^{n}} \\
& c_{j}=\frac{4(1-\theta)}{3\left(\tilde{n}_{i, e}\right)_{j}^{n+1}} \frac{\Delta t}{\left(\xi_{j+1}-\xi_{j-1}\right)\left(\xi_{j}-\xi_{j-1}\right)} \frac{\left(\kappa_{i, e}\right)_{j-1 / 2}}{v_{j}{ }^{n} v_{j-1 / 2}^{n}}
\end{aligned}
$$

The electron temperature equation is modified by the inclusion of the Joule heating term to give 
$D_{j}=D_{j}+\frac{1}{3} \frac{n_{j}}{\mu_{0}^{2}\left(V_{j}^{n}\right)^{2}} \frac{1}{\left(\tilde{n}_{e}\right)_{j}^{n}}\left[\left(\frac{B_{j+1}-B_{j}}{\xi_{j+1}-\xi_{j}}\right)^{2}+\left(\frac{B_{j}-B_{j-1}}{\xi_{j}-\xi_{j-1}}\right)^{2}\right]$

(In the above equations, $v_{j}^{n}$ refers to the relative cell volume before adjustment for pressure balance, while $v_{j}{ }^{n+1}$ refers to the cell volume changed by the factor $y$ to re-establish pressure balance; thus equation (2.57) gives $v_{j}{ }^{n+1}-v_{j}{ }^{n}=(y-1) v_{j}{ }^{n}=\left(1-\frac{1}{y}\right) v_{j}{ }^{n+1}$.) Since the plasma at the wall sees a constant, very small temperature which can be set equal to zero, the boundary conditions on equation (2.55) are

$$
\left(T_{i, e}\right)_{0}=\left(T_{i, e}\right)_{j+1}=0
$$

and the system can be solved by the fast,tridiagonal method [2-15]. The DT reaction, the electron-ion equilibration terms and the electron radiation loss terms are next computed explicitly using an IMSL routine [2-16] which solves ordinary differential equation systems using Runga-Kutta formulas of fifth and sixth order [2-17, 2-18]. The reaction probability shown in figure 1 is fitted with a sixth order polynomial in en $T_{i}$ to give

$$
\begin{aligned}
& \langle\sigma v\rangle=710^{-27} T_{j}{ }^{5.73} \exp \left[-0.410653\left(\ln T_{j}\right)^{2}\right. \\
& -0.18008\left(\text { en } T_{j}\right)^{3}+0.040016\left(\text { en } T_{j}\right)^{4} \\
& \left.-0.00446127\left(\text { \&n } T_{\mathfrak{i}}\right)^{5}+2.82210^{-4}\left(\text { en } T_{j}\right)^{6}\right]
\end{aligned}
$$

where $[T]=\operatorname{keV}$ and $[<\sigma \vee]]=\mathrm{m}^{3} / \mathrm{sec}$. This approximate reaction probability is compared to the actual values in figure 4 .

The alpha heating terms in the electron and ion temperature equations 


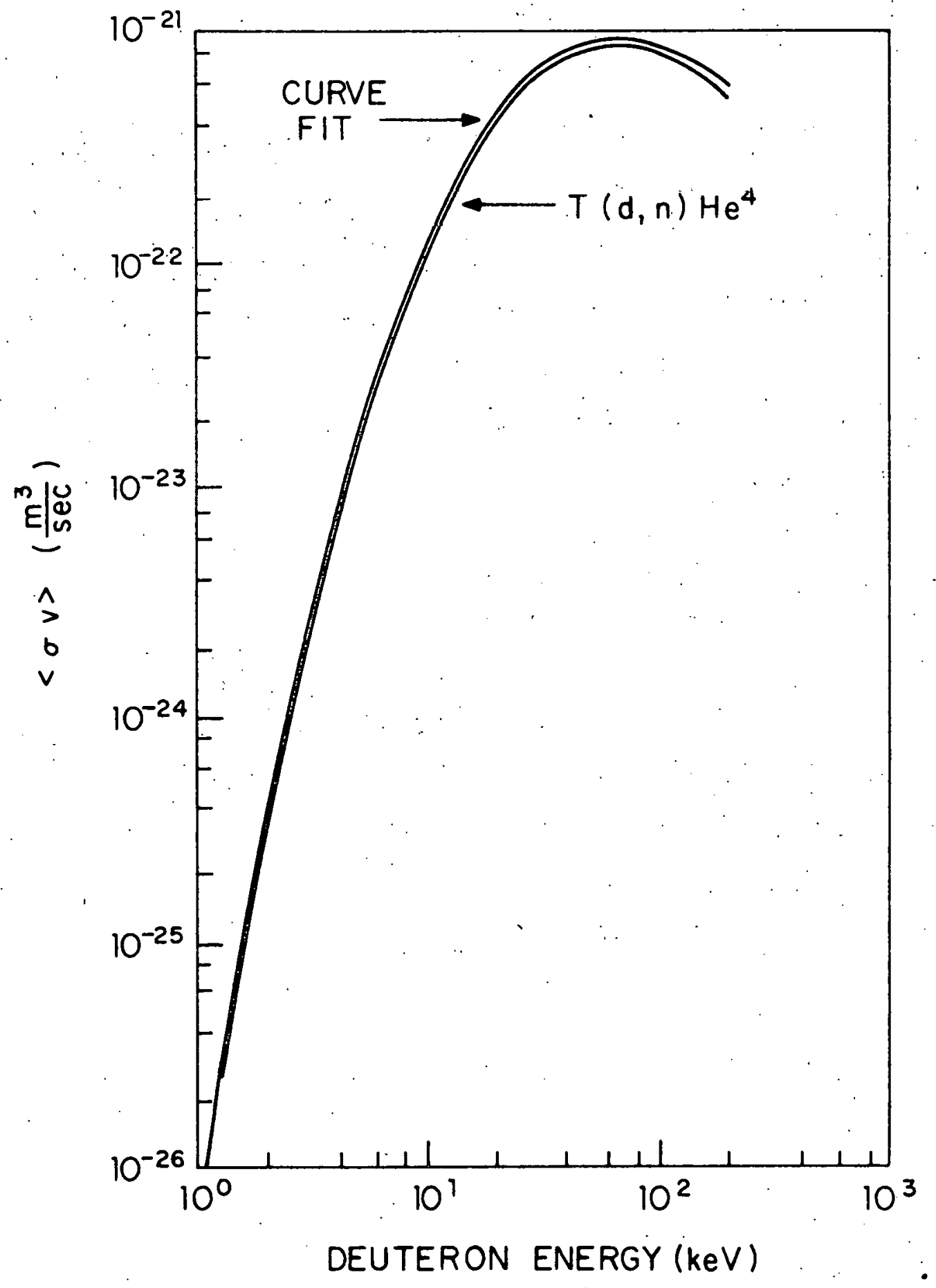

Figure 4 (chapter 2) Curve-fit used for the reaction probability 
are then evaluated explicitly, as described in appendix II, to give

$$
\left(T_{i, e}^{*}\right)_{j}^{n+1}=\left(T_{i, e}^{\prime \prime}\right)_{j}^{n+1}+\frac{2 \Delta t}{3\left(n_{i, e}^{\prime \prime}\right)_{j}^{n+1}} Q_{\alpha i, e}^{j}\left(n_{i, e}^{\prime \prime}, T_{i, e}^{\prime \prime}\right)
$$

where the double prime (") refers to the number densities and temperatures given by the Runga-Kutta solver after reactions, equilibration and radiation have been accounted for, and, as before, the asterisk ( $*$ ) refers to the final temperature values for this time step, to be adjusted for pressure balance at the beginning of the next time step. Since the number densities are affected only by the reaction kinetics and cell compression

$$
\left(n_{i, e}^{*}\right)_{j}^{n+1}=\left(n_{i, e}^{\prime \prime}\right)_{j}^{n+1}
$$

Finally, the magnetic field equation (2.52) gives a finite difference scheme analogous to that for the electron and ion temperatures in equation (2.61),

$$
+A_{j}\left(B^{*}\right)_{j+1}^{n+1}+B_{j}\left(B^{*}\right)_{j}^{n+1}+C_{j}\left(B^{*}\right)_{j-1}^{n+1}=D_{j}
$$

where the coefficients are given by

$$
\begin{aligned}
& A_{j}=\frac{\theta \Delta t}{\left(\xi_{j+1}-\xi_{j-1}\right)\left(\xi_{j+1}-\xi_{j}\right)} \frac{n_{j+1 / 2}}{v_{j}^{n+1} v_{j+1 / 2}^{n+1}} \\
& c_{j}=\frac{\theta \Delta t}{\left(\xi_{j+1}-\xi_{j-1}\right)\left(\xi_{j}-\xi_{j-1}\right)} \frac{n_{j-1 / 2}}{v_{j}^{n+1} v_{j-1 / 2}^{n+1}}
\end{aligned}
$$




$$
\begin{aligned}
& B_{j}=1-A_{j}-c_{j}+\theta \frac{v_{j}^{n+1}-v_{j}^{n}}{v_{j}^{n+1}} \\
& D_{j}=(\tilde{B})_{j}^{n+1}\left[1+a_{j}+c_{j}-(1-\theta) \frac{v_{j}^{n+1}-v_{j}^{n}}{v_{j}^{n}}\right]-a_{j}(\tilde{B})_{j+1}^{n+1}-c_{j}(\tilde{B})_{j-1}^{n+1}
\end{aligned}
$$

and where

$$
\begin{aligned}
& a_{j}=\frac{(1-\theta) \Delta t}{\left(\xi_{j+1}-\xi_{j-1}\right)\left(\xi_{j+1}-\xi_{j}\right)} \frac{n_{j+1 / 2}}{v_{j}^{n} v_{j+1 / 2}^{n}} \\
& c_{j}=\frac{(1-\theta) \Delta t}{\left(\xi_{j+1}-\xi_{j-1}\right)\left(\xi_{j}-\xi_{j-1}\right)} \frac{n_{j-1 / 2}}{v_{j}^{n} v_{j-1 / 2}^{n}}
\end{aligned}
$$

(The factor $\theta$ is set equal to $1 / 2$ in both equation (2.61) and (2.67), to give the Crank-Nicholson scheme.) The wall is assumed to have a very low electrical resistivity, giving

$$
B_{0}=B_{1}=B_{J}=B_{J}+1
$$

Physically, this condition corresponds to [2-12, 2-19] the pressure jump across the plasma-wall interface exactly equaling and canceling the jump in magnetic field. There is then no $j \times B$ force at the wall to cause the plasma to separate from the wall, or to drive into it; there is no $E \cdot j$ Joule heating at the plasma-wall interface.

Because of the boundary condition (2.69), the tridiagonal system given by equation (2.67) connot be solved by the same fast method as the conduction equations used. This tridiagonal system of simultaneous linear algebraic equations is solved by an IMSL routine [2-16] which solves simultaneous algebraic equations using Brown's methods [2-20, 
$2-21]$

The numerical scheme described above has been programmed on a CDC 7600 computer, whose speed allows the use of small time steps (rather than more elaborate fully implicit numerical methods) to insure stability and accuracy. Time steps were usually taken to be $10^{-3} \tau_{b}$, so that several thousand steps were used per run. In a few cases, where the explicit treatment of the electron-ion equilibration led to numerical instabilities, the time step was cut to $10^{-4} \tau_{b}$ and stable solutions resulted. For test cases run with two time step values of $10^{-3} \tau_{b}$ and $10^{-4} \tau_{b}$, final results agreed to better than three percent.

D. Results

The results of the analysis described above are given in this section in the form of plasma temperature, number density and magnetic field profiles. The plasma: motion which maintains the static pressure balance and the induced current is described. The change in total pressure of the plasma is presented, and the effects of alpha particle heating are analyzed:

As a hot plasma is brought into contact with the cold wa11, a thermal boundary layer develops, and the plasma near the wall cools. If thermonumclear reactions are occuring and high energy alpha particles are produced and contained, the plasma can be heated by the energy deposition of these reaction products. All regions of the plasma. radiate energy at varying rates, cooling the electrons and, through electron-ion equilibration, the ions. The temperature profiles at 
different times are shown in figure 5 for a typical plasma undergoing all these processes. (The characteristic time used in this section is lhe bremsstrahiung c0017ng time $\tau_{B}$ given by equation (AI.8), since, unlike the conduction cooling time, it is size-independent.) For short times $\left(t \sim 0.1 \tau_{B}\right)$ the temperature profiles are qualitatively similar to those obtainable from a simple conduction model. The ions having a greater transverse thermal conductivity than the electron gas, because of their larger gyro-radius, lose energy more quickly to the wall than the electrons, and thus near the wall the ion temperature is less than the electron temperature. However, through equilibration, the ions cool the electrons and therefore the electron temperature. near the wall is only slightly greater than the ion temperature. Near the plasma center radiation and alpha particle heating are competing and, at later times $\left(t \sim \tau_{B}\right)$, the results are evident. The ions are heated by alpha particle energy deposition (for background temperatures $\lesssim 30 \mathrm{keV}$ most alpha energy is deposited in the ions) and cooled by thermal conduction losses. The electrons are heated by electron-ion equilibration and cooled by radiation losses. The resulting temperature profiles are smooth, with the center heated by alpha particle energy deposition and the edge cooled by ion thermal conduction, for several radiation cooling times, after which the temperature profiles decay smoothly due to conduction and radiation.

The plasma in figure 5 is just marginally an "ignition" plasma, with some alpha heating contributing to the plasma temperature. The alpha particle number density profiles are shown in figure 6 , where the effect of wall collisions is clearly evident. There is a growing pile up of "cold" thermal alpha particles (whose kinetic energy has been 


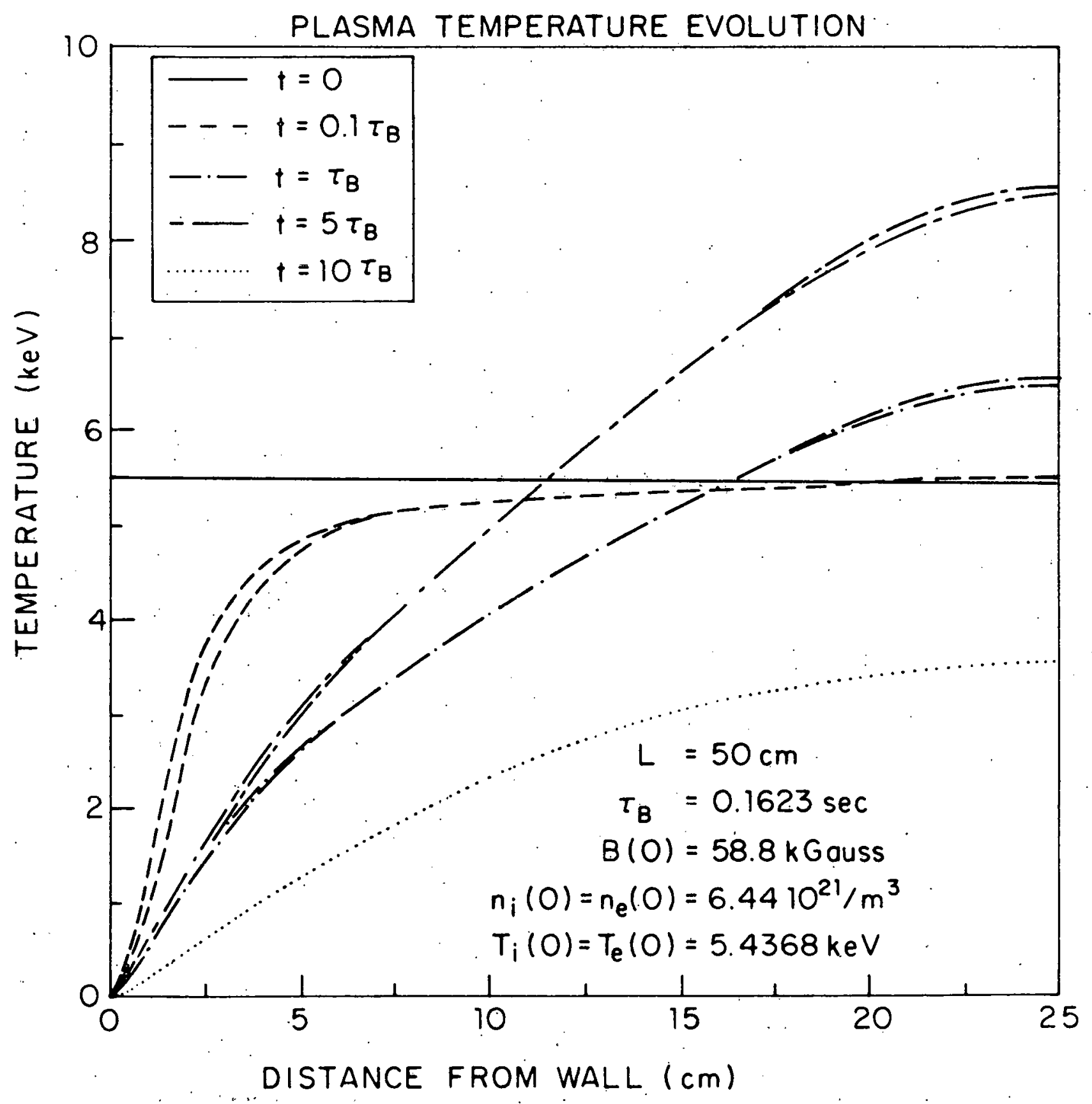

Figure 5 (chapter 2) Spacial profiles of the time evolution of the plasma temperature 


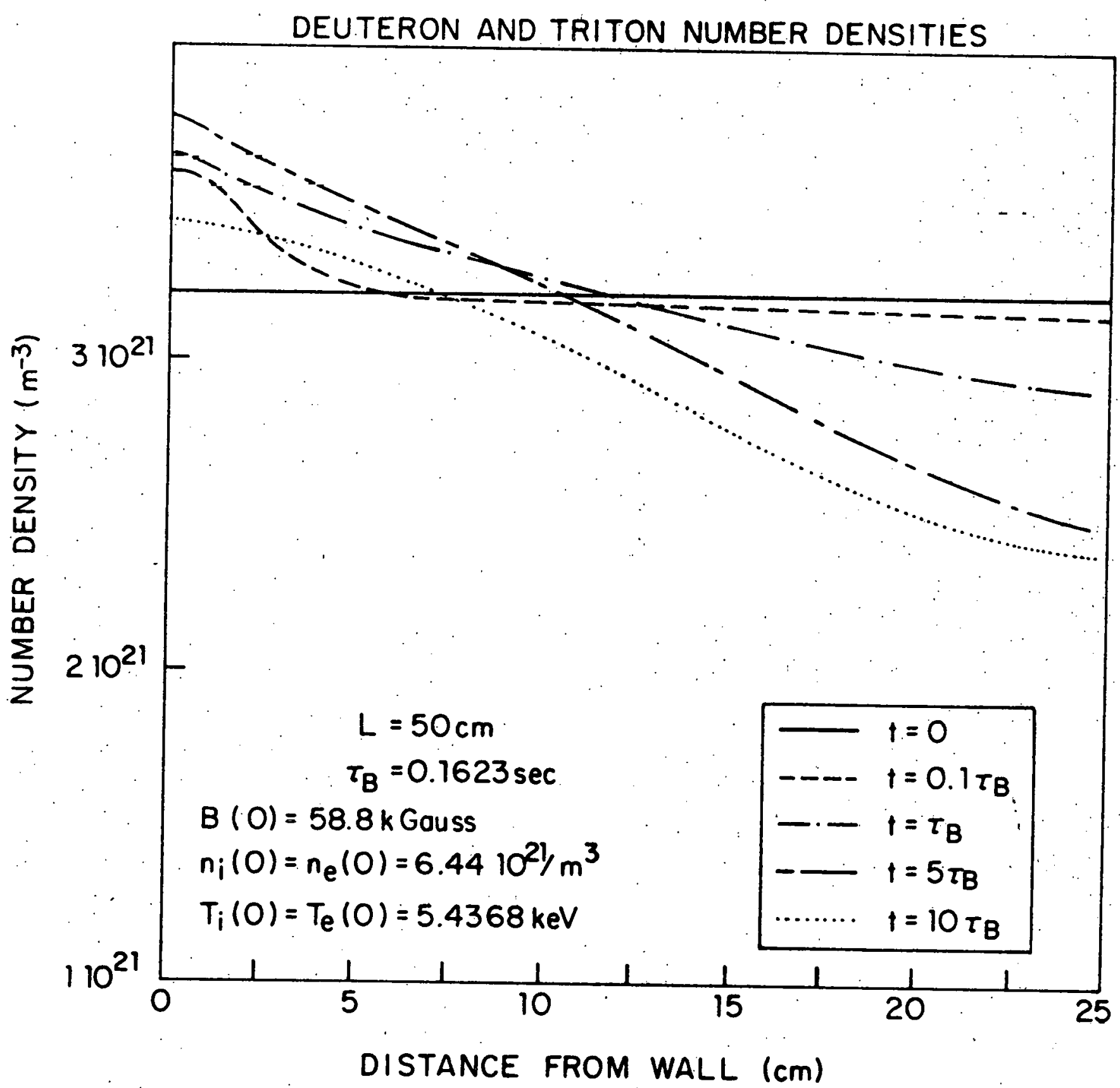

Figure 7 (chapter 2) Spatial profiles of the time evolution of the deuteron and triton number densities (with $n_{D}=n_{T}$ ), which together with the thermalized alpha particles form the ion fluid 
density, gives an indication of the magnitude of the alpha particle wall collision effect.

The relative cell volumes which give explicitly the effects of the plasma pressure-driven convection are shown in figure 8. Initially ( $t \sim 0.1 \tau_{B}$ ) there is a very sharp compression near the wa.ll with a corresponding expansion of the central plasma. At later times. $\left(t i \tau_{B}\right)$ the compression is almost uniformly distributed across the plasma, with the central region expanding and the edge being compressed, and at still later times $\left(t \gtrsim 10 \tau_{B}\right)$, as the plasma cools completely, the compression and expansion relaxes and the plasma again approaches a uniform state.

The convective motion, described above, of the plasma motion transverse to the magnetic field creates an electric field and corresponding electric currents; which in turn generate a magnetic field. The magnetic field profiles are shown in figure 9, and are qualitatively similar to those obtainable from a frozen flux model. Again, at very short times $\left(t \sim 0.1 \tau_{B}\right.$ ) the magnetic flux is increased sharply near the wall because of convection and "approximately" frozen flux. Magnetic diffusion tends to decrease the magnetic field gradient at later times $\left(t \sim \tau_{B}\right)$, but alpha particle heating drives the central plasma to expand and thus tends to increase the magnetic field gradient. The result is the establishment of a magnetic "well" at the center of the plasma, with smoothly increasing fields toward the walls, which incidentally improves both the alpha particle confinement and the thermal insulation.

The electric currents associated with these magnetic field profiles are shown in figure 10. At early times $\left(t \sim 0.1 \tau_{B}\right)$ there is a strong 


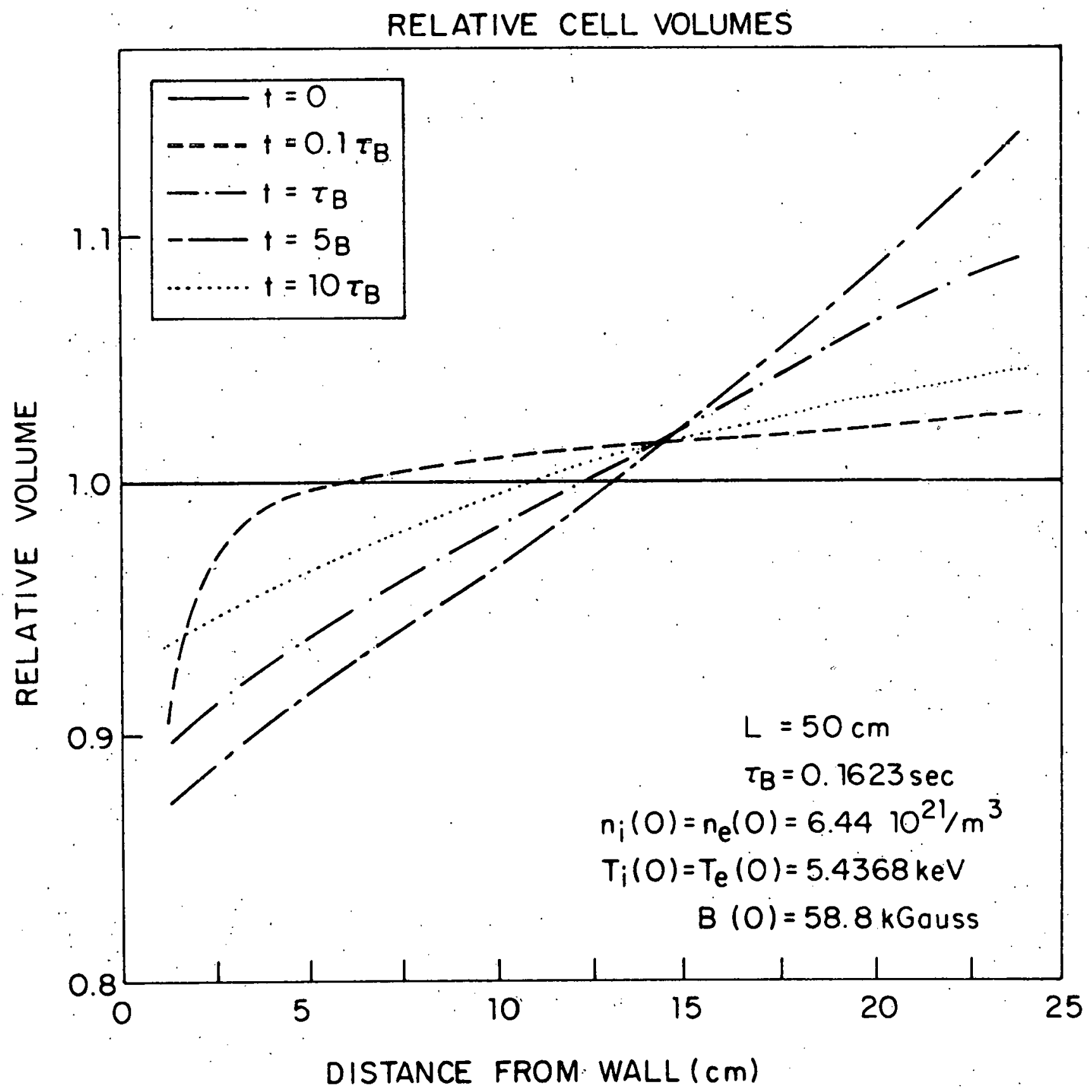

Figure 8 (chapter 2) Spatial profiles of the time evolution of the relative cell volumes 
IMBEDDED MAGNETIC FIELD

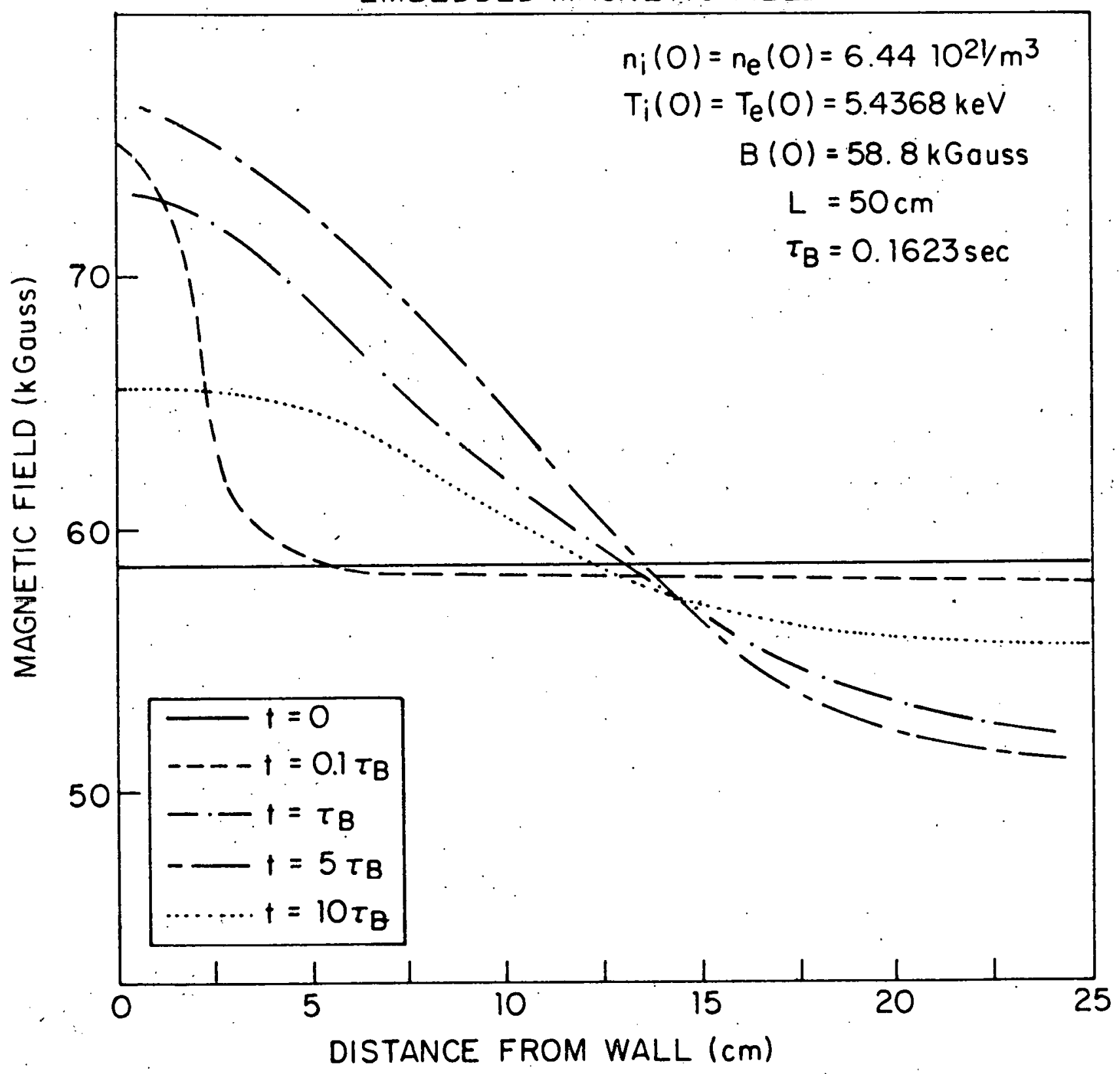

Figure 9 (chapter 2) Spatial profiles of the time evolution of the transverse imbedded" magnetic field 
ELECTRIC CURRENT

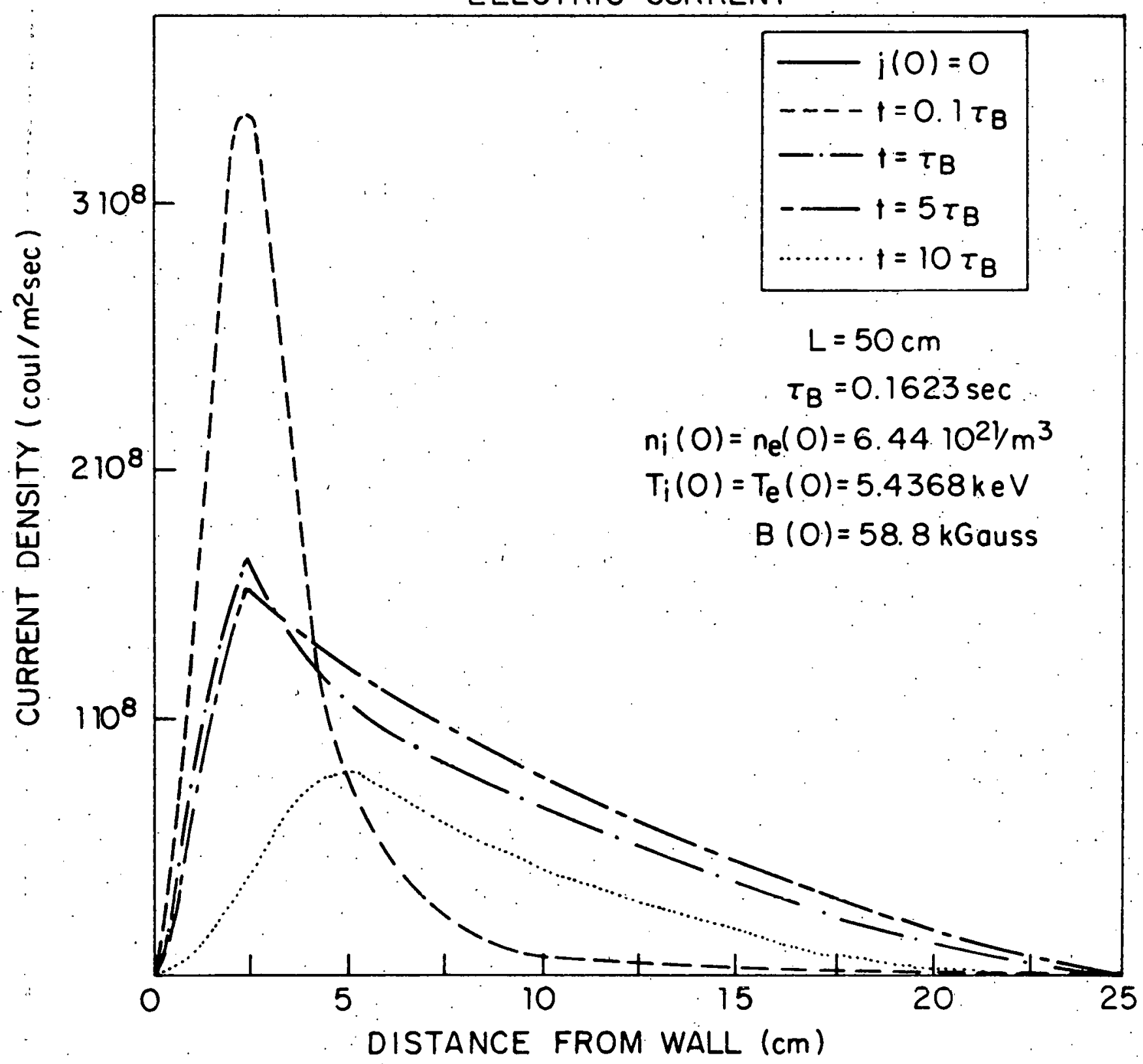

Figure 10 (chapter 2) Spatial profiles of the time evolution of the induced electric currents 
current flowing near the wall, while at later times $\left(t \lambda_{i} \tau_{B}\right)$ the electric current decreases as the magnetic field gradient smooths.

The bulk motion of the plasma is shown in the velocity profiles in figure 11. At short times $\left(t \sim 0.1 \tau_{B}\right.$ ) the plasma flows toward the walls (negative velocities) as the center is heated and the edges cool, while at later times $\left(t>\tau_{B}\right)$ the plasma is almost quiescent. As the plasma finally. cools down $\left(t \geq 10 \tau_{B}\right.$ ), it begins to flow back towards the center (positive velocities) re-establishing almost uniform magnetic fields and temperatures. This convective motion $\left(u \lesssim 10^{-1} \mathrm{~m} / \mathrm{sec}\right)$ is very much slower than the magnetoacoustic speed $\left(c_{\mathrm{m}} \approx 10^{6} \mathrm{~m} / \mathrm{sec}\right)$.

The pressure balance maintained by the plasma motion described above is shown in figure 12, as a function of time. This system pressure is equivalent to the pressure the walls are supporting. In the plasma described above (with $L=50 \mathrm{~cm}$ ), the alpha heating just balances the radiation and conduction losses to maintain an almost constant system pressure for an extended period of time $\left(t \lesssim 7-8 \tau_{B}\right)$ before the losses dominate and the total pressure decays. The system pressures are also shown for two other cases studied, where the plasma initial configuration is unchanged and only the distance separating the two walls is varied: When the walls are close enough that all the alpha particles collide with the wall before heating the plasma $\left(L=20 \mathrm{~cm} \sim \mathrm{ra}_{\max }(3.5 \mathrm{MeV})\right)$ the total pressure simply decays as the conduction and radiation losses dominate. Conversely when the walls are far enough apart $(L=1 \mathrm{~m})$ that less than $40 \%$ of the alpha particle orbits can intersect the walls, the alpha particle heating can dramatically increase the system pressure by almost an order of magnitude before the inevitable decay begins to dominate. For very short times (on the order of a few alpha slowing down or 


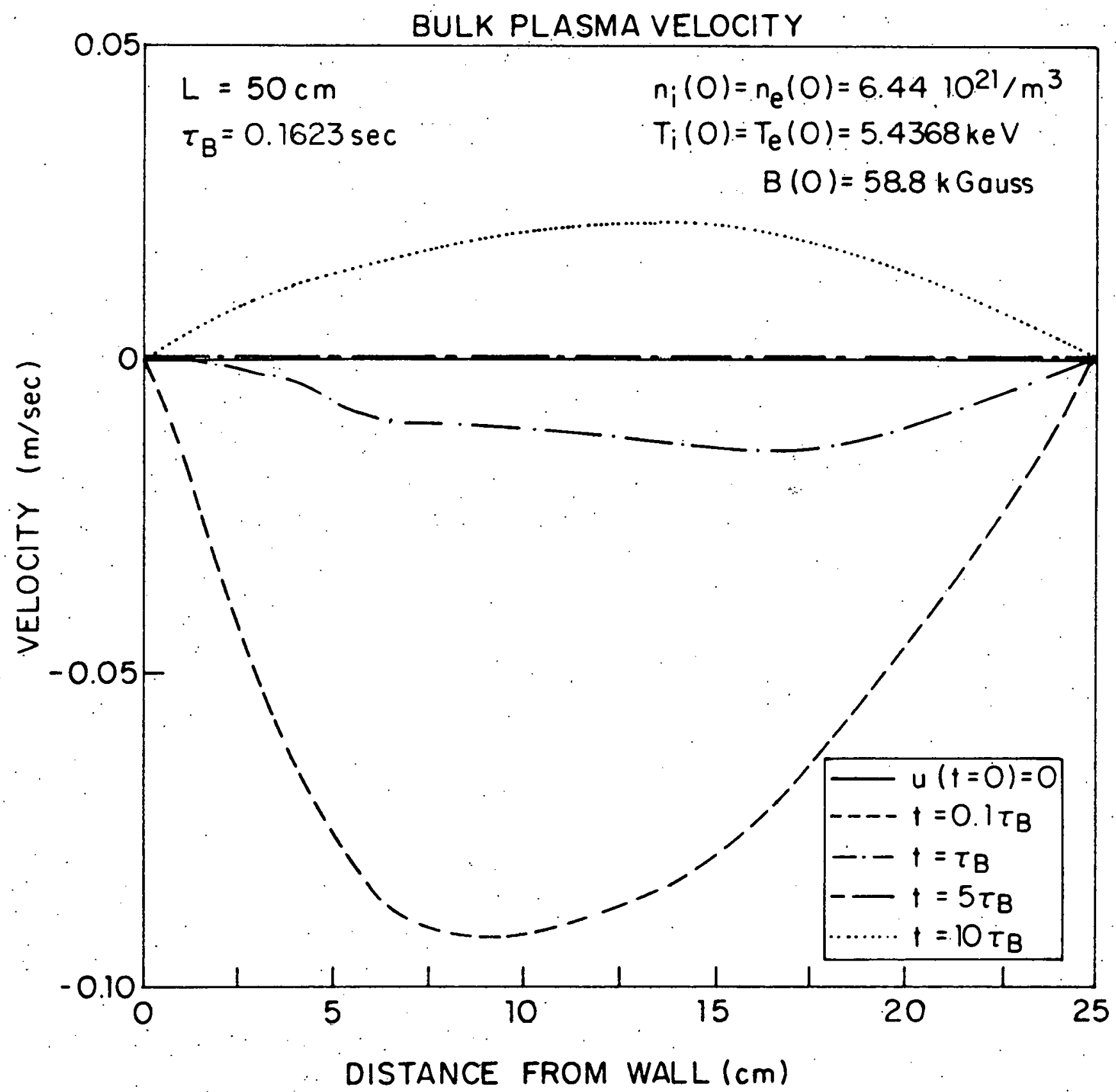

Figure 11 (chapter 2) Spatial profiles of the time evolution of the bulk plasma velocity, where negative values correspond to flow toward the walls and positive values to flow away from the walls 


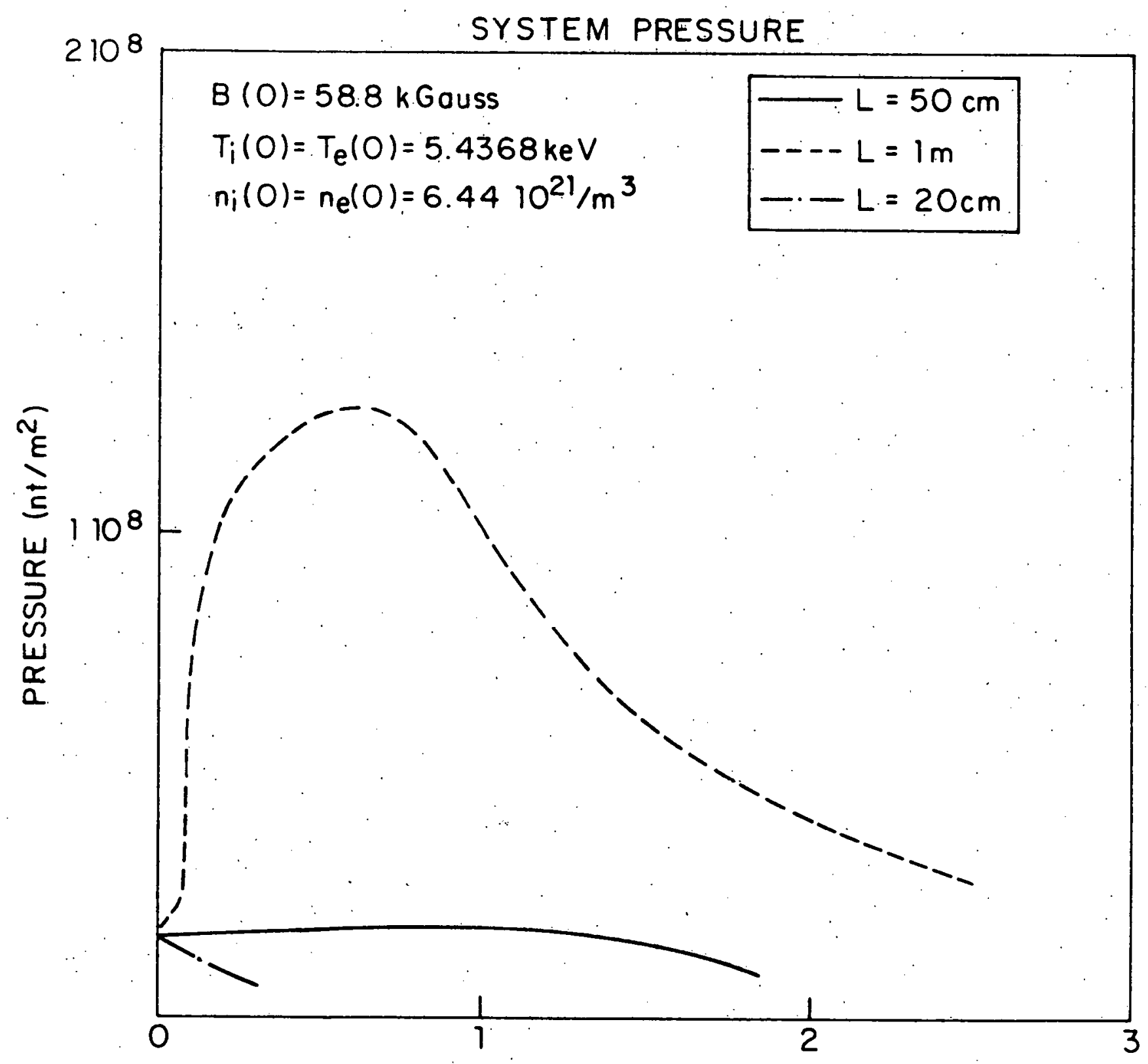

TIME (sec)

Figure 12 (chapter 2) Time evolution of the total system pressure (kinetic and magnetic) for various wall separation distances. 
thermalization times), the pressure rise is gradual; after the alpha particles have time to slow down in the background plasma the pressure increases sharply as the ion and electron temperatures rise.

These temperatures are shown in figure 13, for a plasma whose initial conditions are identical to those of the plasma described in figures 5 through 11 , and whose distance between the walls is $L=1 \mathrm{~m}$ rather than $L=50 \mathrm{~cm}$ as before. At short times $\left(t \lesssim 0,1 \tau_{B}\right)$ the temperature profiles are very similar to those in figure 5 . The ion temperature is slightly lower than the electron temperature near the wall due to the greater ion thermal conductivity. At later times ( $t$ $\tau_{B}$ ) the ion temperature near the wall remains lower than the electron temperatures, while, in the central plasma, the alpha particles deposit most of their energy to the electrons (since the background temperature is now $230 \mathrm{keV}$ ) with the ion temperature rising also due mainly to electron-ion equilibration but also to some alpha heating. At still later times $\left(t \sim 5 \tau_{B}\right.$ ) the radiation terms cool the electron fluid sufficiently to cause the ion temperature in the central region to overtake the electron temperature, and ion conduction and electron radiation then continue to $c 001$ the plasma. The temperature profiles, when compared with those of figure 5 , show a wide thermal boundary layer with sharp temperature gradients insulating a central hot core with almost uniform temperature, rather than the very smooth temperature gradients seen before. The central plateau region corresponds to those parts of the plasma where the alpha particles are perfectly contained.

The associated alpha particle number densities are shown in figure 14. The increased compression of the plasma near the wall and the corresponding expansion of the hot central region cause the alpha 


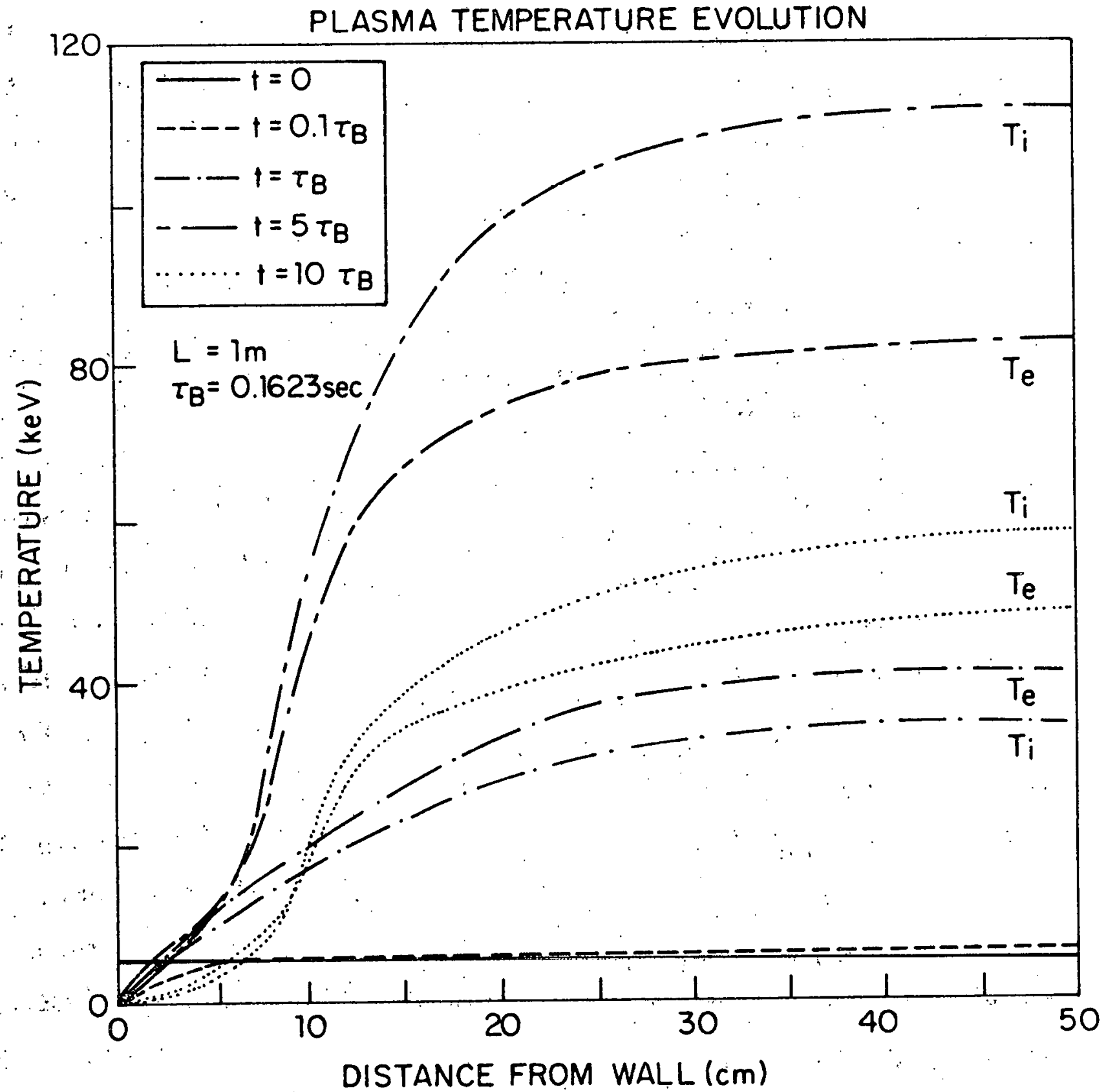

Figure 13 (chapter 2) Spacial profiles of the time evolution of the plasma. temperature 


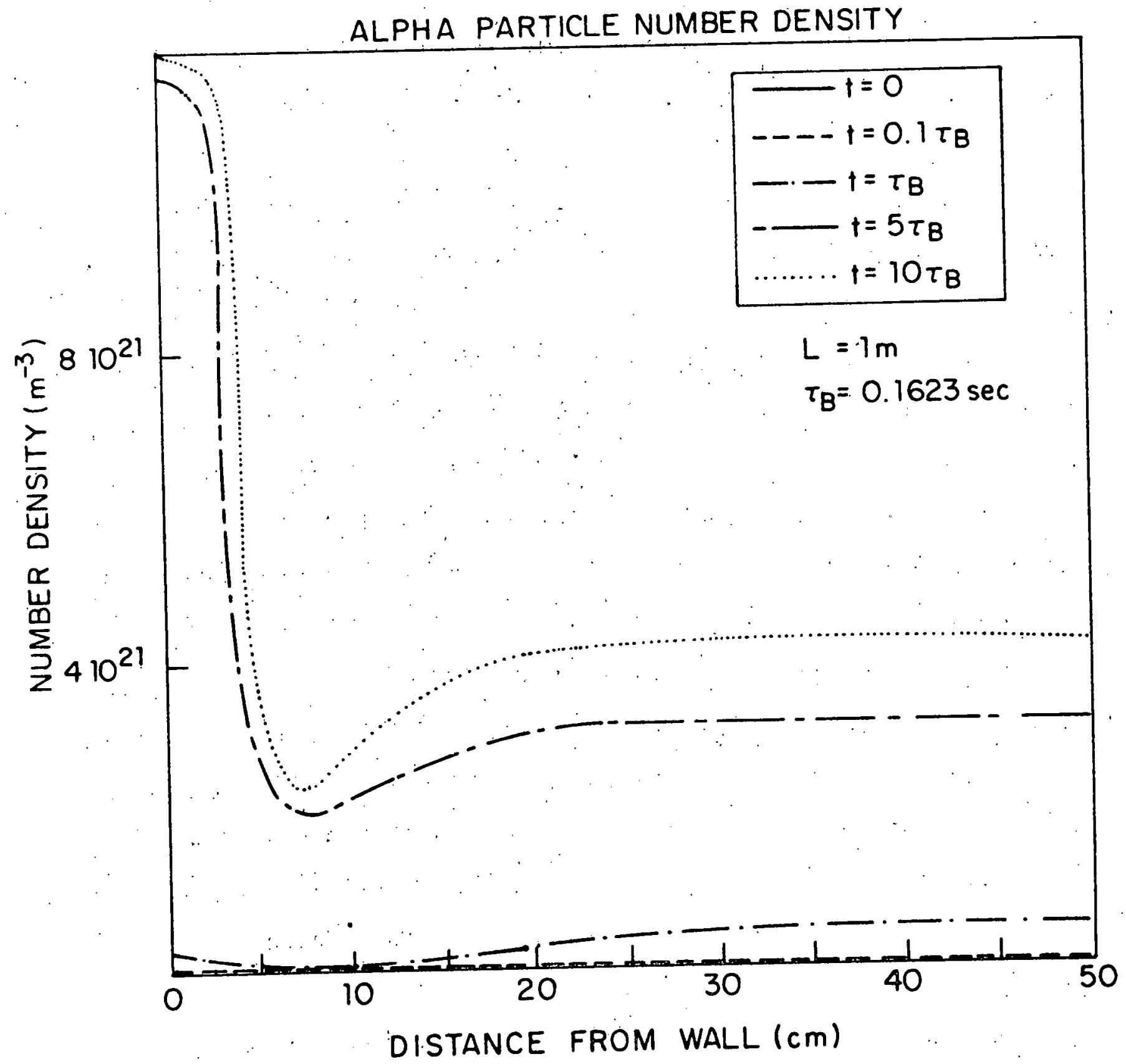

Figure 14 (chapter 2) Spatial profiles of the time evolution of the total alpha particle number density (thermalized and non-thermalized) 
particle number density near the wall, increased by the pile-up due to wall collisions, to rise by an additional factor of 2 . Most of the profile shows the plateau followed by a concave downward region indicative of total alpha orbit containment. Less than $40 \%$ of the plasma is affected by alpha particle wall-collisions, while in the alpha particle number density profiles shown in figure 6 almost $80 \%$ of the plasma is affected by wall collisions.

For comparison, the temperature and alpha particle number density profiles corresponding to the third $(L=20 \mathrm{~cm})$ plasma system pressure shown in figure 12 are given in figures 15 and 16 . The plasma again has identical initial conditions to those described above, but the distance between the walls is now $L=20 \mathrm{~cm}$. Since the gyroradius of a $3.5 \mathrm{MeV}$ alpha particle in a $58.8 \mathrm{kGauss}$ magnetic field is $i 9.2 \mathrm{~cm}$, all of the plasma is affected by the occurrence of alpha particle-wall collisions, and thus very little of the alpha particle energy present is deposited in the ion or electron fluid. The ion temperature near the wall is again always less than the electron temperature due to the greater ion thermal conduction losses, while in the central region the electron temperature is always less than the ion temperature due to the radiation loss terms. The alpha particle number density profiles as expected show a tremendous pile-up of alpha particles next to the wall, and an almost uniformly depleted profile throughout the rest of the plasma.

The results given in this section show the importance of accurate modelling of alpha particle energy deposition in problems of wall-confined thermonuclear plasmas. The plasma temperature evolution is very dependent on the spatial containment of the alpha particle orbits, and 


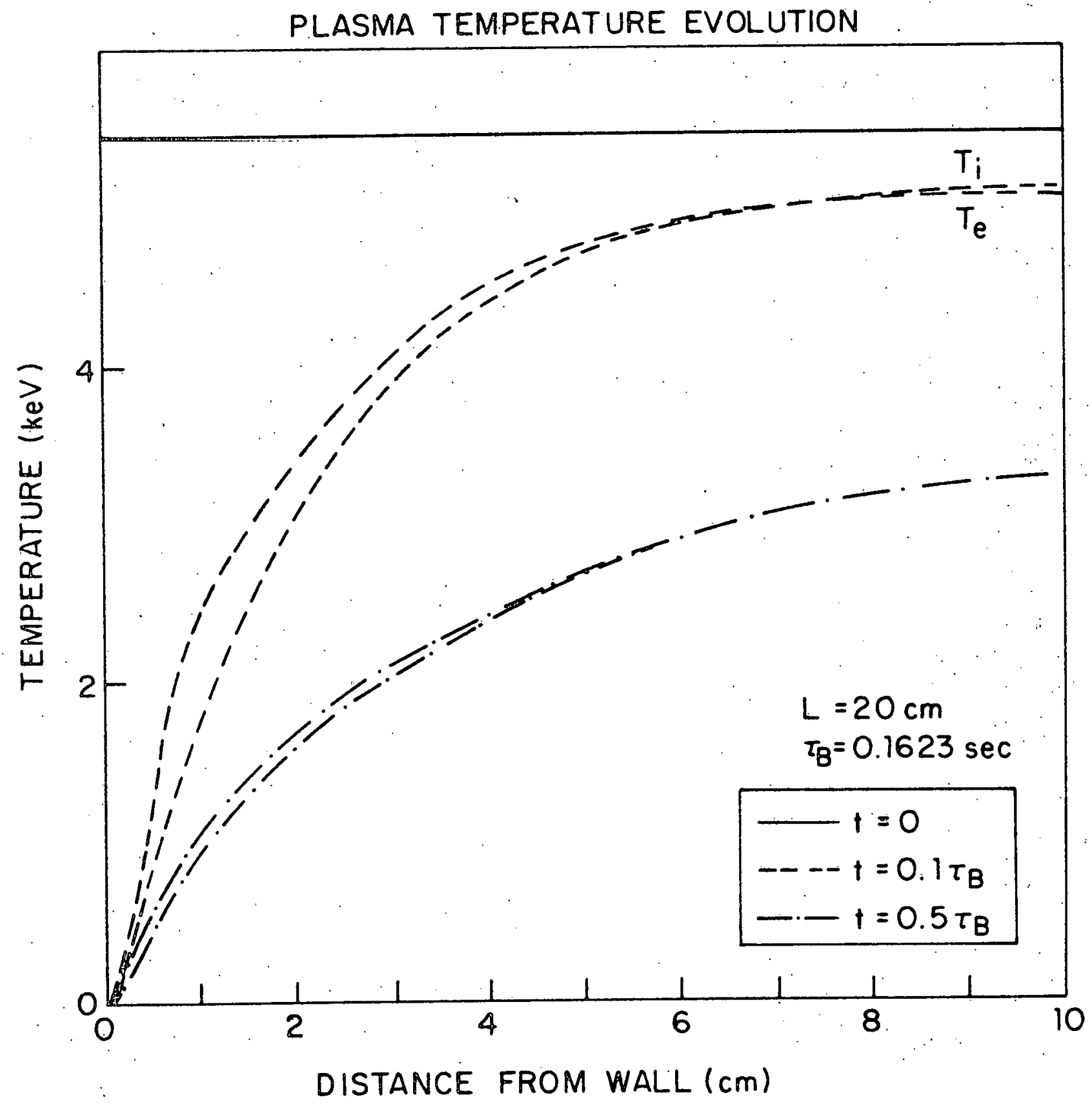

Figure 15 (chapter 2) Spatial profiles of the time evolution of the plàsma temperature 


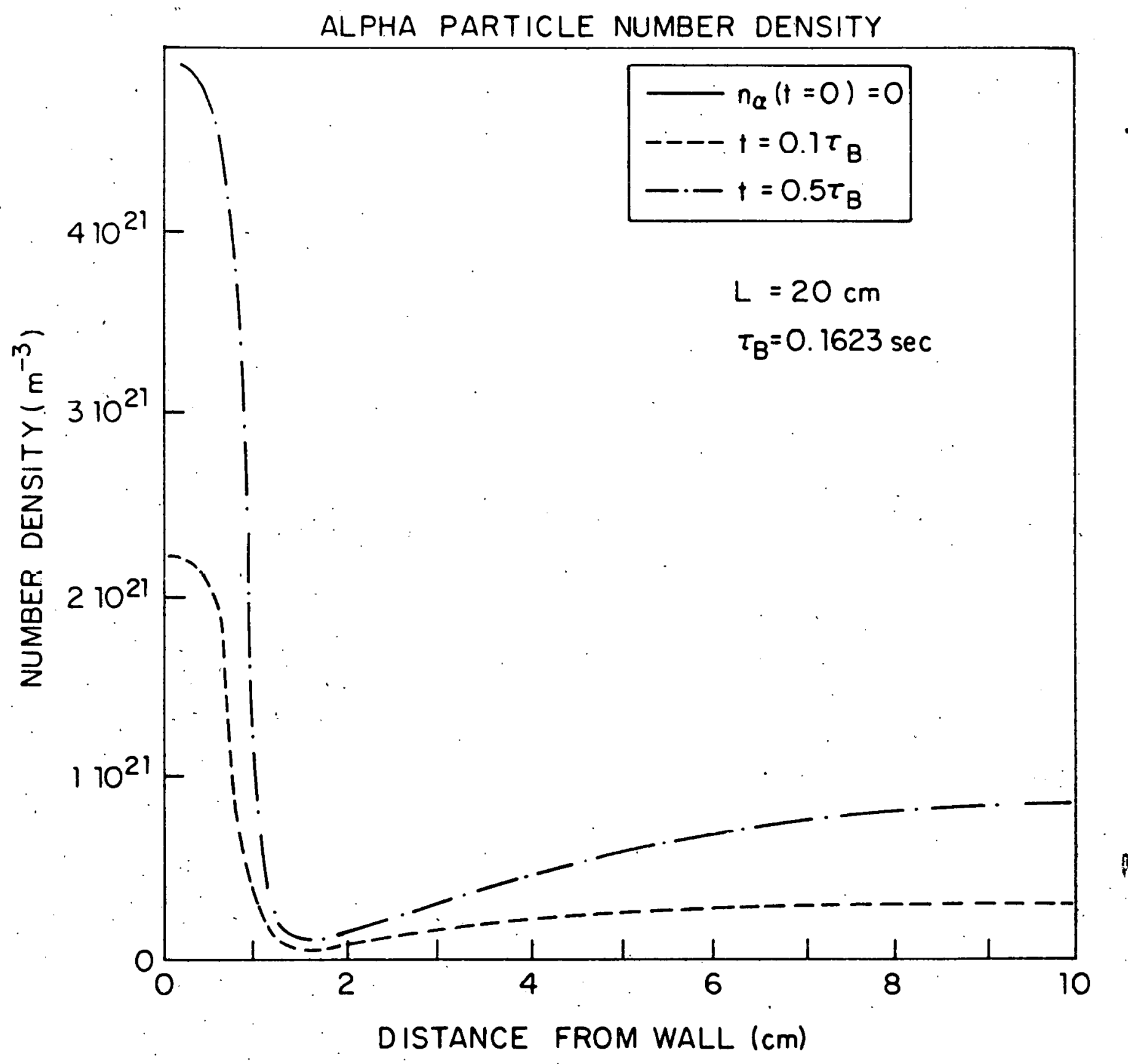

Figure 16 (chapter 2) Spatial profiles of the time evolution of the total alpha particle number density (thermalized and nonthermalized) 
also dependent (although less so) on the time-behavior of the energy deposition. The accuracy and importance of the alpha particle model is discussed in appendix II. The accuracy of the rest of the program can be, and has been, tested by comparing results with the earlier work done on plasma boundary layers by M.S. Chu [2-22] and B. Jensen [2-23] as wel.1 as the current work being done by I, Lindemuth $[2-24,2-25]$.

By suppressing thermonuclear reactions and setting the ion mass to that of hydrogen, the results given by Jensen for a fully ionized boundary layer can be recovered, with the results agreeing to better than $10 \%$. Setting the wa 11 pressure constant and radiation terms to zero as well allows comparison with M.S. Chu's semi-infinite plasma boundary layer results, again to better than $10 \%$. Suppressing only thermonuclear reactions by setting $\langle\sigma v\rangle=0$ allows comparison with Lindemuth's ANIMAL code [2-26], an Eulerian code which does not assume pressure balance but solves the full velocity equation. The results agree to within $20 \%$, with the greatest difference occurring near the wall and being partly attributable to the difference in the number of cells used. 
Chapter 3: Energy Gains in a Shock-heated Wall-confined Fusion Reactor

The results of the one-dimensional plasma-wall problem studied in the previous chapter can be used to analyze the shock-heated wallconfined fusion cycle described in the introduction. In this chapter, the energy multiplication is first calculated for the simple limiting cases of "total burn" (where all the fuel is consumed) and radiationcooled burn (where both alpha particle heating and thermal conduction losses are neglected). The energy gain is then calculated for the general one-dimensional problem, in which alpha particle and Ohmic heating, radiation and conduction cooling, magnetic diffusion and convection-maintained pressure balance are present. Since the insulating magnetic field will cause the alpha particles to drift to the walls very quickly in cylindrical geometry (due to grad $B$ and curvature. drifts), and since two-dimensional boundary layer instabilities may greatly increase thermal conduction losses, the energy multiplication is also calculated for modified versions of the one-dimensional problem, in which alpha particle heating is suppressed and the thermal conductivity enhanced.

A. Definition of Energy Gain and Calculation of Maximum Possible Energy Gain

One measure of the energy multiplication of a fusion cycle is the ratio of its energy output, in the form of both radiation and kinetic 
energy of the reaction products, to the energy input required to establish both the initial plasma temperature and initial magnetic field configuration. If the fusion energy output is defined as the number of fusion events occurring (which for the DT reaction is equivalent to the number of either alpha particles or neutrons produced) multiplied by the kinetic energy of the fusion reaction products (3.5 MeV for the alpha particles and $14.1 \mathrm{MeV}$ for the neutrons resulting from a DT reaction), the energy gain is given by

$$
Q=\frac{E_{r a d}+E_{f}}{E_{j n}}=1+\frac{E_{f}}{E_{i n}}
$$

where it is assumed that all the input energy can be recovered in the form of radiation as the plasma cools, even in the absence of any fusion events, and that all the charged particle kinetic energy as well as that of the neutrons is perfectly recoverable. The actual energy radiated out is greater than the input energy due to alpha particle heating of the plasma, but the kinetic energy of the alpha particles themselves is correspondingly reduced, so that equation (3.1) is still valid. No energy is assumed permanently lost to, or "stored in", the wall due to either thermal heating by the plasma or by particle collisions such as those occurring when a gyro-orbit intersects the wall.

The maximum possible energy multiplication for any fusion cycle corresponds of course to the total consumption of the fuel, and is given by

$$
E_{f}=17.6(\mathrm{MeV}) n_{\alpha} V
$$


where $V$ is the total volume of the plasma. The specific energy output is then given by

$$
e_{f}=2.8210^{-12} \cdot n_{1}
$$

since the number of alpha particles present after all the DT fuel is consumed is equal to the number of either deuterons or tritons initially present, which equals the number of molecules in the preionized gas.

(Unless otherwise noted, the units used are $[E]=\mathrm{J},[\mathrm{e}]=\mathrm{J} / \mathrm{m}^{3}$, $[\mathrm{n}]=\mathrm{m}^{-3}$ and $[\mathrm{T}]=\mathrm{keV}$. )

The conservation equations for the shock-heating and re-expansion phases are solved in appendix III to calculate specific power inputs. The energy per unit volume required to shock-heat a plasma to a desired temperature $T_{6}$, calculated from strong shock limits for a linear geometry, is given by equation (AIII.22) as

$$
\begin{aligned}
e_{f} & =10^{7} \frac{p_{1} \text { (mtorr) }}{T_{1}\left({ }^{\circ} \mathrm{K}\right)} T_{6} \\
\therefore \quad & =1.0310^{-15} n_{1} T_{6}
\end{aligned}
$$

and, with the constant corrected to fit the calculated exact results for linear geometry given by equation (AIII.19), this expression becomes

$$
e_{f}=9.17 \cdot 10^{-16} n_{1} T_{6}
$$

Thus the maximum possible energy multiplication as given by equation 
(3.1) is

$$
\begin{aligned}
Q & =1+\frac{2.8210^{-12} n_{1}}{9.1710^{-16} n_{1} T_{6}} \\
& =1+\frac{3071}{T_{b}}
\end{aligned}
$$

As would be expected, the maximum energy gain decreases with increasing initial temperature, since the total energy output is fixed while more and more energy input is required to heat to higher initial temperature.

B. Optimization of Energy Gain in the Presence of Radiation Cooling

At low initial temperatures the plasma will cool off long before any significant burn occurs. The primary loss mechanism, because it is size-independent, is radiation cooling. Small systems will see no alpha particle heating throughout most of the plasma, because the gyro orbits of the high energy alphas will not be contained but will intersect the walls. Large systems will not see thermal conduction losses throughout much of the plasma, since thermal boundary. layers will insulate large regions of the central plasma. However, radiation is emitted by all parts of the plasma, regardless of system size.

A simple model of a one-temperature, radiation-cooled DT plasma is given by

$$
\frac{\partial n_{\alpha}}{\partial t}=-\frac{\partial n_{D}}{\partial t}=-\frac{\partial n_{T}}{\partial t}=n_{D} n_{T}<\sigma v>
$$




$$
\begin{gathered}
\frac{3}{2}\left(n_{i}+n_{e}\right) \frac{\partial T}{\partial t}=-4.4610^{-21} n_{e}\left(n_{D}+2 n_{\alpha}\right) T^{\frac{1}{2}} \\
n_{i}=n_{D}+n_{T}+n_{\alpha}
\end{gathered}
$$

These equations are a limiting case of the more general one-dimensional problem of chapter 2, in which the alpha heating, thermal conduction and electron-ion equilibration terms have all been set to zero. The boundbound and free-bound radiation terms are also neglected since they do" not become significant until the temperature drops far below $1 \mathrm{keV}$, where fusion events have essentially stopped occurring. This system of equations can be solved on a desktop calculator, and the resulting energy gains can be compared to those given by computer calculations in which the alpha heating and thermal conduction terms were set to zero. A comparison of these results, together with the associated alpha particle number densities and burn fractions, for various values of the initial temperature $\mathrm{T}_{6}$, are given in the following table:

\begin{tabular}{lllll}
$T_{6}(\mathrm{keV})$ & $\mathrm{n}_{\alpha}\left(\mathrm{m}^{-3}\right)$ & $\mathrm{f}(\%)$ & \multicolumn{1}{c}{$Q_{\text {comp }}$} & \multicolumn{1}{c}{$Q_{\text {calc }}$} \\
\hline 2.1644 & $1.6410^{17}$ & .005 & 1.073 & 1.112 \\
3.4304 & $1.8910^{18}$ & .059 & 1.527 & 1.524 \\
5.4368 & $1.58 .10^{19}$ & 0.49 & 3.80 & 3.78 \\
8.6168 & $9.4610^{19}$ & 2.94 & 11.45 & 11.16 \\
13.657 & $3.6710^{20}$ & 11.4 & 26.64 & 25.70 \\
21.644 & $8.9310^{20}$ & 27.8 & 40.5 & 38.6 \\
34.304 & $1.4810^{21}$ & 46.2 & 42.4 & 40.0 \\
54.368 & $1.9310^{21}$ & 60.6 & 35.3 & 33.8 \\
86.168 & $2.2610^{21}$ & 70.1 & 26.0 & 23.6
\end{tabular}


The less than $10 \%$ discrepancy in the energy multiplication is attributable to the electron-ion equilibration term; when it is included, the ion temperature falls somewhat slower so the net burn is slightly higher. (The time step on both computer and calculator was $10^{-3} \tau_{B}$, and the calculation was halted when the temperature dropped below $1 \mathrm{keV}$. )

The maximum possible energy. gain, given by equation (3.4), and the radiation-controlled energy gain given in the above table are shown in figure 1. The "radiation-only" energy multiplication peaks at an initial temperature of about $30 \mathrm{keV}$, as expected from the characteristic time curves, since at this temperature the bremsstrahlung cooling time given by equation (AI.8) and the DT reaction time given by equation (AI.9) are approximately equal.

The energy gain has been studied here as a function of the initial plasma temperature and, in the next section, as a function of the size of the reaction chamber. Other parameters are the magnetic field, which in appendix III is determined as a function primarily of the plasma temperature and number density (and of the initial fill magnetic field). The specific power and energy inputs are shown in appendix III to be proportional to the initial number density, and the maximum energy output given by equation (3.2) is also directly proportional to the number density, so that the maximum possible energy multiplication is essentialiy. independent of number density. Since the major characteristic times given in appendix I (electron-ion equilibration, alpha slowing down, conduction and bremsstrahlung cooling, and DT reaction times) are all inversely proportional to the number density, the gains in alpha heating 


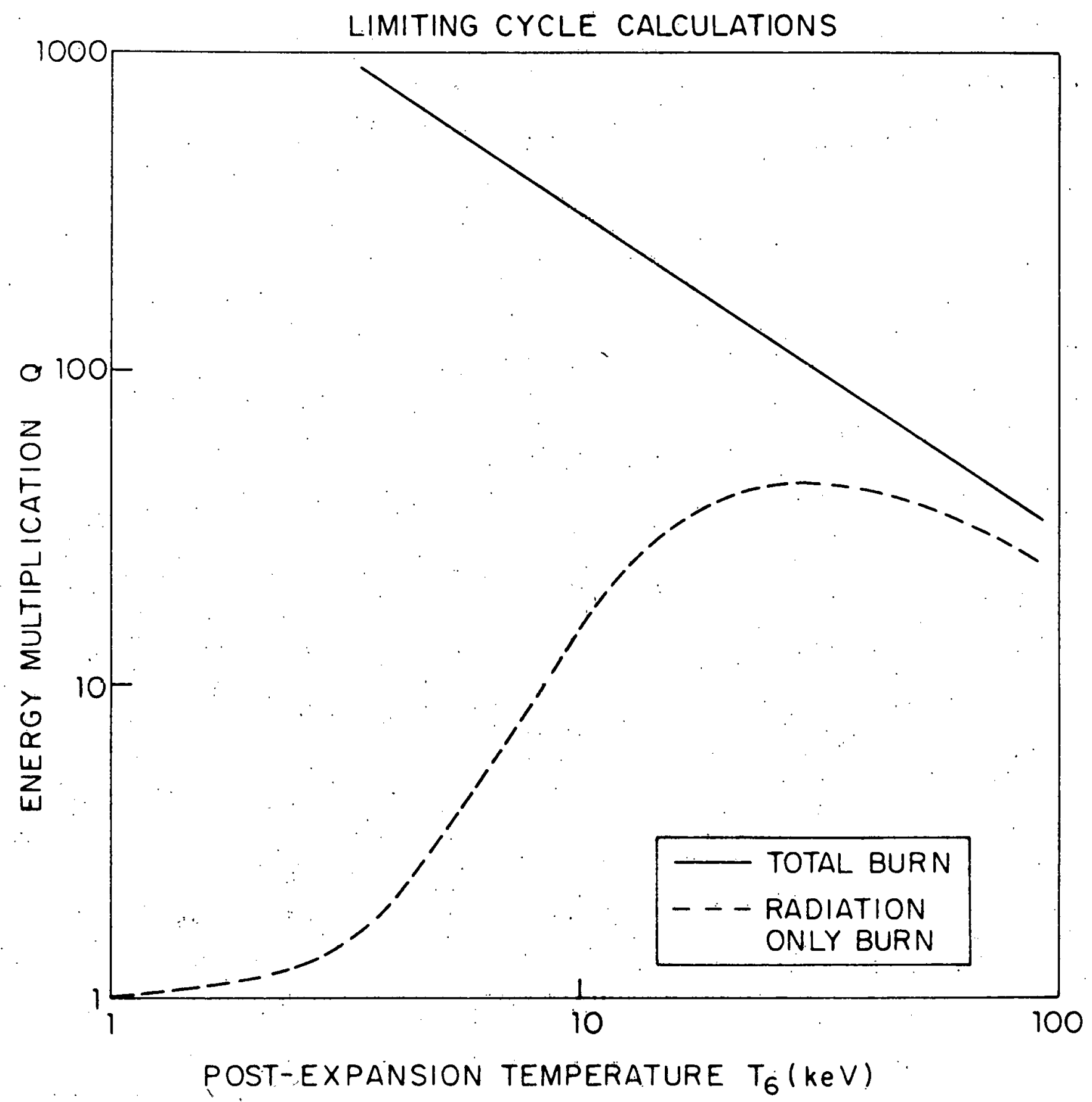

Figure 1. (chapter 3) Limiting energy multiplication factors for a shock-heated wall-confined fusion reactor. 
and DT reaction rates due to increasing number densities are balanced by enhanced radiation and conduction losses, with no great net discernable effect. This can be confirmed by studying the energy gain of the simple radiation-governed plasma for different initial number densities, given in the following table:

$T_{6}(k e v)$

$\mathrm{Q}\left(\mathrm{n}_{\mathrm{O}}=\right.$

$Q\left(n_{0}=\right.$

$Q<\mathrm{n}_{0}=$

$\left.3.2210^{20} / \mathrm{m}^{3}\right)$

$3.2210^{21} / \mathrm{m}^{3}$ )

$\left.6.4410^{21} / \mathrm{m}^{3}\right)$

5.4368

3.78

3.78

3.78

13.657

25.89

25.89

25.89

34.304

40.25

40.25

40.25

86.168

24.73

24.73

24.73

Thus any possible optimization of the energy multiplication due to varying the initial number density would be a second-order effect compared to the optimization possible by varying the initial temperature or chamber size. However, the magnetic field is shown in appendix III to be proportional to the square root of the number density, and increasing the magnetic field both improves the alpha particle confinement and decreases the thermal conduction losses, so some improvement in energy gain is possible by increasing the initial fill number density.

C. Energy Gains from the General One-dimensional Model

The cases studied thus far are unaffected by the presence or absence of a wall, and hence unaffected by the reaction chamber size. 
The wall is considered here to act in only two ways -- as a constant low-temperature heat șink governing thermal conduction losses, and as an obstacle to the alpha particle gyration and therefore an alpha particle energy loss mechanism, while its effect as a source of impurities and the presence of a boundary layer such as that studied by Jensen are neglected. The calculation of energy multiplication factors for the general one-dimensional problem of chapter 2, with thermal losses and alpha particle heating included, allow a study of the parametric effect of the system size, but first the linear temperature and number density profiles calculated must be related to the cylindrical cycle geometry described in chapter 1 (and shown there in figure 1). With the dimension $x$ identified as the distance from the wall, the differential volume associated with any variable $g(x)$ is shown in figure 2. The energy output

$$
E_{f}=2.8210^{-12} \iint 2 \pi r n_{\alpha}(r, z) d r d z
$$

is then, for the spatial cell structure described in chapter 2, given by

$$
\begin{aligned}
E_{f} & =2.8210^{-12} \sum_{j=1}^{10} 2 \pi n_{\alpha}^{j}\left\{( L - x _ { j } ) \left[\left(f_{2} L-x_{j-1}\right)^{2}-\left(f_{2} L-x_{j}\right)^{2}+\left(f_{1} L+x_{j}\right)^{2}\right.\right. \\
& \left.\left.-\left(f_{1} L+x_{j-1}\right)^{2}\right]+\left(x_{j}-x_{j-1}\right)\left[\left(f_{2} L-x_{j}\right)^{2}-\left(f_{1} L+x_{j}\right)^{2}\right]\right\}
\end{aligned}
$$

The energy gains for different values of $L$ (with $f_{1}=1$ and $f_{2}=2$ ), calculated from the energy input given by equation (AIII.18) and the fusion energy output found by substituting the number. density profiles computed 


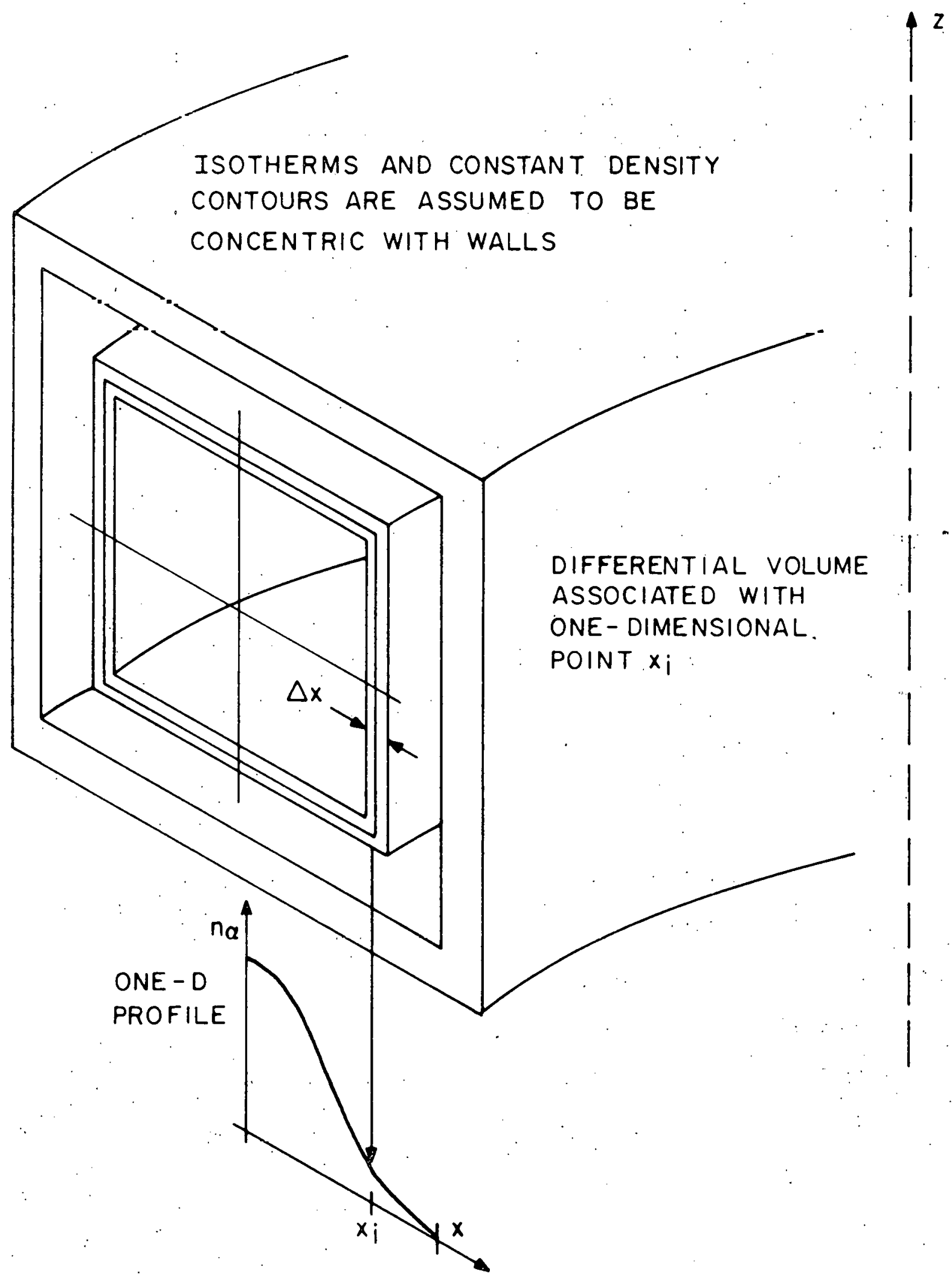

Figure 2 (chapter. 3) Integration volume for a one-dimensional profile in the coaxial reaction chamber 
in chapter 2 into equation (3.6), are shown in figure 3 . The equivalent burn fractions are shown in the following table, where they are compared to those of the simple radiation-cooled case described above:
$\mathrm{T}_{6}(\mathrm{keV})$
$f(L=20 \mathrm{~cm})$
$f(L=50 \mathrm{~cm})$
$f(L=\operatorname{lm})$
$f$

with alpha heating, radiation and conduction radiation only

$\begin{array}{lllll}2.1644 & 0.0006 \% & 0.002 \% & 0.003 \% & 0.005 \% \\ 3.4304 & 0.008 & 0.03 & 0.05 & 0.06 \\ 5.4368 & 0.09 & 5.2 & 64.8 & 0.49 \\ 8.6168 & 0.78 & 36.6 & 79.4 & 2.9 \\ 13.657 & 5.1 & 56.5 & 81.1 & 11.4 \\ 21.644 & 27.1 & 74.2 & 82.4 & 27.8 \\ 34.304 & 49.1 & 77.2 & 83.3 & 46.2 \\ 54.368 & 62.7 & 80.2 & 83.6 & 60.6 \\ 86.168 & 72.0 & 81.9 & 83.8 & 70.1\end{array}$

At low temperatures where no significant alpha heating occurs, because very few alpha particles are produced, all the results (both energy multiplication and burn fraction) are lower than those of the radiation-governed case of equations (3.5), as expected due to the additional thermal conduction losses. As the system size increases and a smaller fraction of the alpha particle orbits intersect the walls, ignition occurs for lower initial temperatures. If the characteristic length is small $(\mathrm{L}=20 \mathrm{~cm})$, the alpha heating merely balances the thermal conduction losses, while for bigger systems the alpha particle energy deposition clearly dominates over the various loss mechanisms. For 


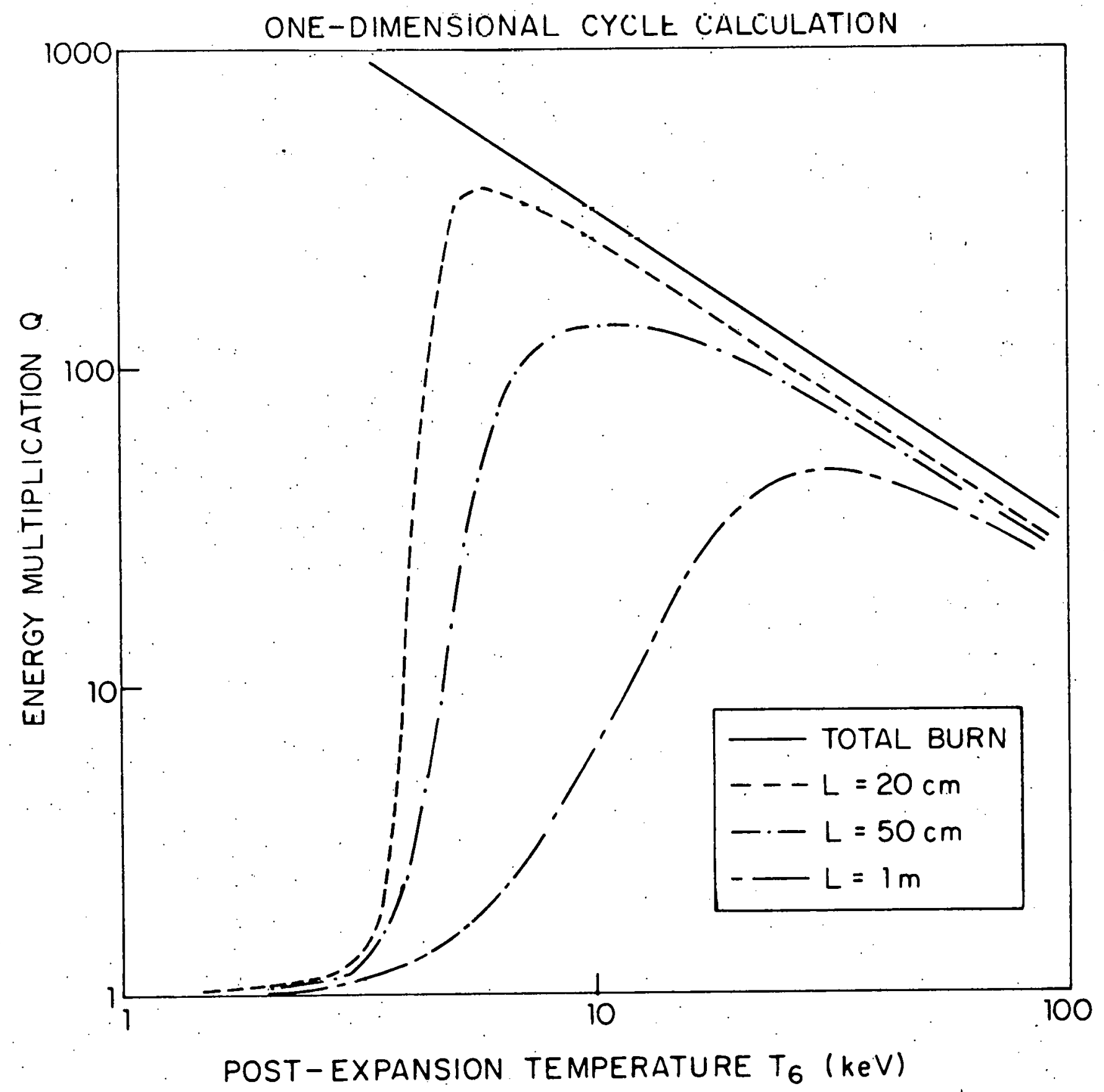

Figure 3 (chapter 3 ) Energy multiplication factors calculated from the one-dimensional results of chapter 2 
"post-ignition" initial temperatures the burn fraction and net energy output tend to approach limiting values, slightly lower than those for total fuel consumption. The energy multiplication is monotonically increasing to a limiting value as system size is increased for a constant initial temperature, but shows a definite peak as the initial temperature is varied for a fixed characteristic length. As the system size is increased, the peak energy gain occurs at progressively lower temperatures, attesting to the effects of ignition.

D. Energy Gains in the Absence of Alpha Particle Heating and the Presence of Enhanced Thermal Conduction

The one-dimensional problem studied so far (with pressure balance maintained by convection during radiation and conduction cooling, Ohmic and alpha particle heating, and magnetic diffusion) overestimates the energy gains possible because it does not take into account several important loss mechanisms which are inherently two-dimensional in nature -- the alpha particle drift in the curved B field of cylindrical coordinates, which substantially increases the rate of wall collisions, and the possiblity of boundary layer instabilities which greatly enhance thermal conduction losses by convecting central hot plasma to the wall in cells. While a full study of these loss mechanisms involves a twodimensional time-dependent problem in cylindrical geometry, their effects can be estimated by simple modifications of the one-dimensional problem.

The effect of the alpha particle drift can be estimated based on the characteristic drift and slowing down times given by equations (AI.12) 
and (AI.6). The energy lost by an alpha particle to the plasma, before it drifts into and collides with the wall, is approximately

$$
\begin{aligned}
\Delta E_{\alpha}(\mathrm{keV}) & =E_{\alpha}(\mathrm{keV})\left[1-\exp \left(-\frac{{ }^{\tau} D}{\tau_{\alpha}}\right)\right] \\
& =3520\left[1-\exp \left(-1.13610^{-26} \frac{e^{2} \mathrm{Bn \ell n \Lambda}}{\left.\left.T^{3 / 2}\right)\right]}\right.\right.
\end{aligned}
$$

and is shown for varying characteristic lengths and background plasma temperatures in the following table:

$\mathrm{T}_{6}(\mathrm{keV})$

$$
\Delta E_{\alpha}(L=20 \mathrm{~cm}) \quad \Delta E_{\alpha}(L=50 \mathrm{~cm}) \quad \Delta E_{\alpha}(L=1 \mathrm{~m}) \quad \Delta E_{\alpha}(L=10 \mathrm{~m})
$$

$\begin{array}{ccccc}2.1644 & 0.56 & 3.49 & 27.84 & 1153.38 \\ 3.4304 & 0.37 & 2.30 & 9.17 & 808.49 \\ 5.4368 & 0.24 & 1.51 & 6.04 & 555.84 \\ 8.6168 & 0.16 & 0.99 & 3.95 & 374.05 \\ 13.657 & 0.10^{\circ} & 0.65 & 2.58 & 248.99 \\ 21.644 & 0.07 & 0.42 & 1.69 & 164.67 \\ 34.304 & 0.04 & 0.28 & 1.10 & 108.44 \\ 54.368 & 0.03 & 0.18 & 0.72 & 71.04 \\ 86.168 & 0.02 & 0.12 & 0.47 & 47.39\end{array}$

For the system sizes studied here $(L \leq 1 \mathrm{~m})$, the potential energy loss is always less than $0.8 \%$ of the total alpha particle energy and is in practice negligible, since only those alpha particles born in the center of the system will lose even that amount of energy. For larger dimensions $(L \geq 10 \mathrm{~m})$, the alpha particle energy loss can be as high as $33 \%$, and thus remains a significant factor. The effect of the alpha particle. 
drift can therefore be included in the general one-dimensional problem, for the characteristic lengths considered here, by simply setting the alpha heating terms to zero.

To examine the effects of enhanced thermal conduction losses due to two-dimensional wall-induced instabilities (discussed in chapter 1) the problem is also studied with the electron and ion thermal conductivities enhanced by factors of 10, 100 and 1000, while the alpha heating terms are still. set to zero. The results of these alpha drift-enhanced conduction studies are shown in figure 4,5 and 6 for three different characteristic lengths $(20,50$ and $100 \mathrm{~cm}$, respectively), and the earlier results of figure 3 are included for comparison.

For small systems $(L \leq 20 \mathrm{~cm})$ the presence or absence of alpha particle heating does not change the energy multiplication greatly, as is seen in figure 4 , since so many gyro-orbits naturally intersect the walls. Thus very little alpha particle energy is left for plasma heating, even without drift losses. The peak energy gain when alpha heating is suppressed does occur at a higher initial temperature than that where the maximum energy multiplication occurs in the presence of alpha heating. The peak for suppressed alpha heating also occurs at a higher initial temperature than that where the radiation-dominated energy gain shown in figure 1 has its maximum, showing the effect of the added conduction losses. The burn fractions obtained in these alpha driftenhanced conduction studies are shown in the following table for various initial plasma teimperatures, for a characteristic length of $20 \mathrm{~cm}$ : 


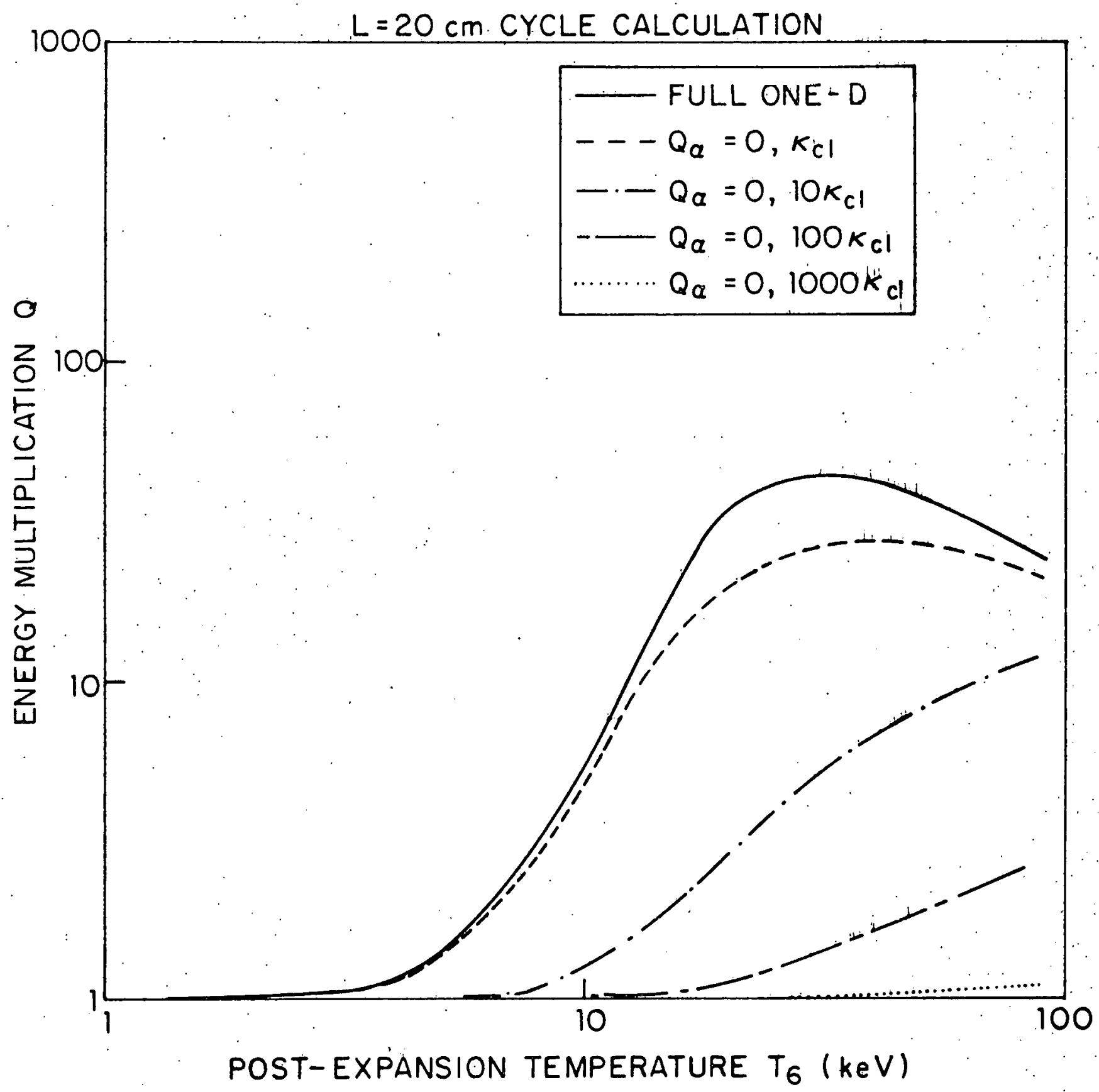

Figure 4 (chapter 3) Energy multiplication factors for a $\operatorname{small}(L=20 \mathrm{~cm})$ system after various two-dimensional loss mechánisms have been taken into account 


\begin{tabular}{lllll}
$\mathrm{T}_{6}(\mathrm{keV})$ & \multicolumn{5}{c}{$f(\%), \mathrm{L}=20 \mathrm{~cm}$} \\
\hline 2.1644 & 0.0005 & $2.810^{-5}$ & $<10^{-6}$ & $<10^{-6}$ \\
3.4304 & 0.007 & 0.0005 & $3.410^{-5}$ & $<10^{-6}$ \\
5.4368 & 0.087 & 0.006 & 0.0004 & $3.510^{-5}$ \\
8.6168 & 0.71 & 0.048 & 0.004 & 0.0004 \\
13.657 & 3.95 & 0.32 & 0.026 & 0.003 \\
21.644 & 13.7 & 1.56 & 0.13 & 0.012 \\
34.304 & 29.6 & 5.47 & 0.52 & 0.048 \\
54.368 & 46.2 & 13.7 & 1.66 & 0.15 \\
86.168 & 60.2 & 31.2 & 4.62 & 0.40
\end{tabular}

Any enhancement in thermal conductivity causes these small systems to become conduction-dominated, with correspondingly low energy gains, as seen in figure 4 . The peak energy multiplication occurs at progressively greater high initial temperatures, and the value of the peak energy gain becomes increasingly small as the enhancement factor grows.

In intermediate size systems $(2 \sim 50 \mathrm{~cm})$ the suppression of alpha particle heating can dramatically reduce the energy multiplication except at high $(T \geq 50 \mathrm{keV})$ initial temperatures, as shown in figure 5 , and again moves the location of the peak energy gain to higher initial temperatures. The energy multiplications for classical conduction begin to approach those of the purely radiation-governed case described by equation (3.5), and large enhancements of the thermal conductivity are needed before the system becomes conduction-dominated. Reasonable gains are easily achieved 


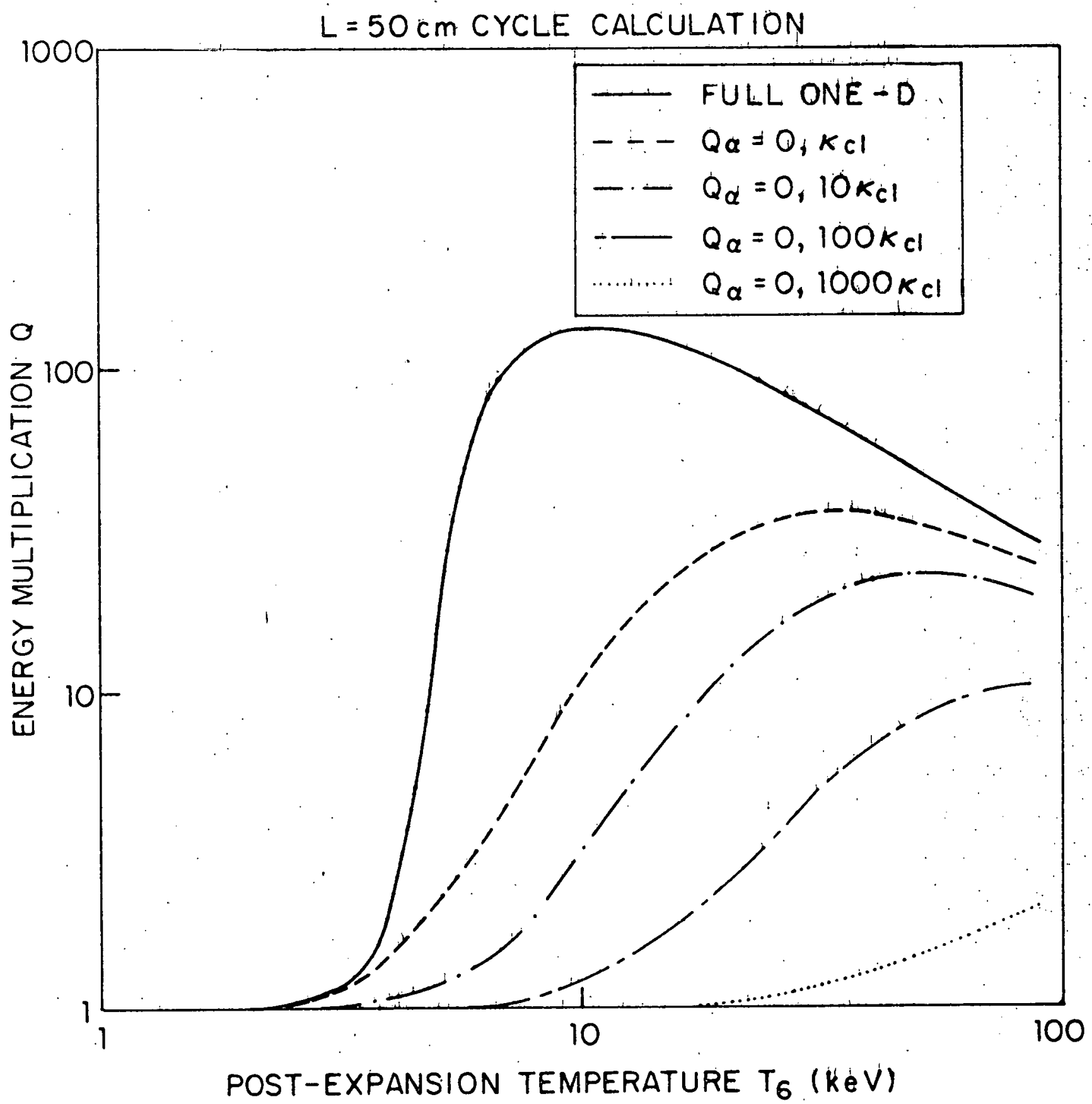

Figure 5 (chapter 3) Energy multiplication factors for an intermediate $(L=50 \mathrm{~cm})$ system after various two-dimensional loss mechanisms have been taken into account 
for realistic conduction increases $\left(k=10\right.$ or $100 \kappa_{\mathrm{cl}}$ ), even in the absence of any alpha particle heating. The equivalent burn fractions for a $50 \mathrm{~cm}$ chäracteristic length are given in the following table:

\begin{tabular}{|c|c|c|c|c|}
\hline $\mathrm{T}_{6}(\mathrm{keV})$ & $K=\kappa_{c l}$ & $\begin{array}{l}, L=50 \mathrm{~cm} \\
K=10 \kappa_{\mathrm{cl}}\end{array}$ & $\kappa=100 \kappa_{c l}$ & $\kappa=1000 \kappa_{c l}$ \\
\hline 2.1644 & 0.002 & 0.0008 & $1.4 \quad 10^{-5}$ & $10^{-6}$ \\
\hline 3.4304 & 0.025 & 0.005 & 0.0003 & $2.210^{-5}$ \\
\hline 5.4368 & 0.26 & 0.047 & 0.004 & 0.0003 \\
\hline 8.6168 & 1.74 & 0.36 & 0.035 & 0.002 \\
\hline 13.657 & .. 7.65 & 2.05 & 0.25 & 0.016 \\
\hline 21.644 & 21.0 & 8.12 & 1.28 & 0.081 \\
\hline 34.304 & 38.7 & 21.2 & 4.85 & 0.31 \\
\hline 54.368 & 54.9 & 38.4 & 13.3 & 0.99 \\
\hline 86.168 & 66.7 & 53.2 & 26.7 & 2.66 \\
\hline
\end{tabular}

At low enhancement factors the burn fractions have begun to approach limiting values.

Larger systems $(L \geq 1 \mathrm{~m})$ are drastically affected by the loss of alpha particle energy confinement due to drift-induced wall collisions, except at the very highest initial temperatures studied (T 100keV), as seen in figure 6 . However, the effect of increased thermal conduction losses due to two-dimensional wall-induced boundary layer instabilities is greatly reduced from that seen in small $L 220 \mathrm{~cm}$ systems. Although the thermal boundary layer increases in thickness as the enhancement factor rises, the hot central plasma is far enough from the wall to be relatively 


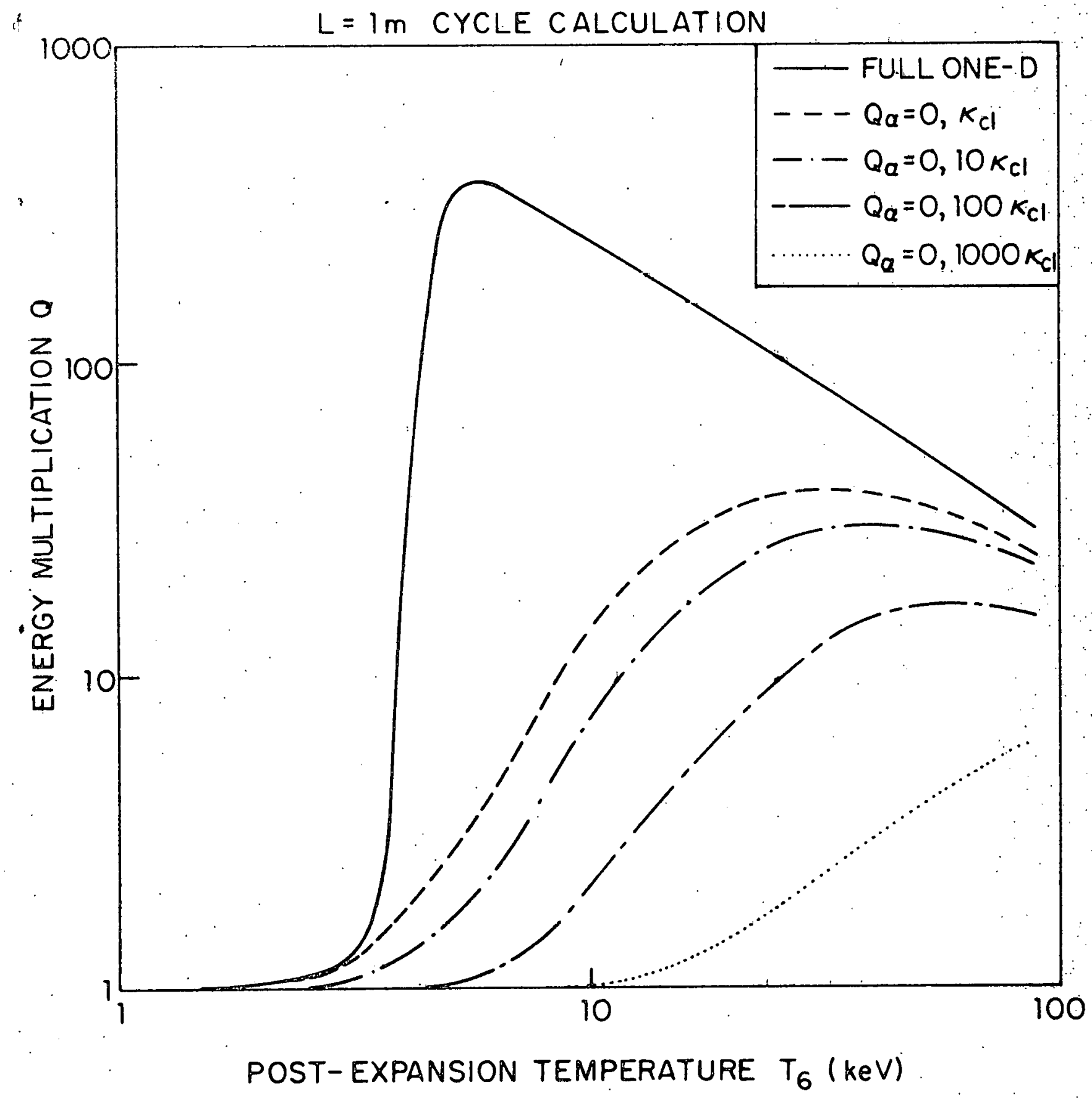

Figure 6 (chapter 3) Energy multiplication factors for a large ( $L=1 \mathrm{~m}$ ) system after various two-dimensional loss mechanisms have been taken into account 
insulated from the increased heat losses by the thermal boundary layer in these large systems. The energy multiplication for perfect classcial thermal conduction is only $2-20 \%$ less than that of the purely radiationcontrolled system, and even conduction enhancements of 1000 show reasonable energy gains, although they require very high initial temperatures. The equivalent burn fractions for a characteristic length of $1 \mathrm{~m}$ are shown in the following table:

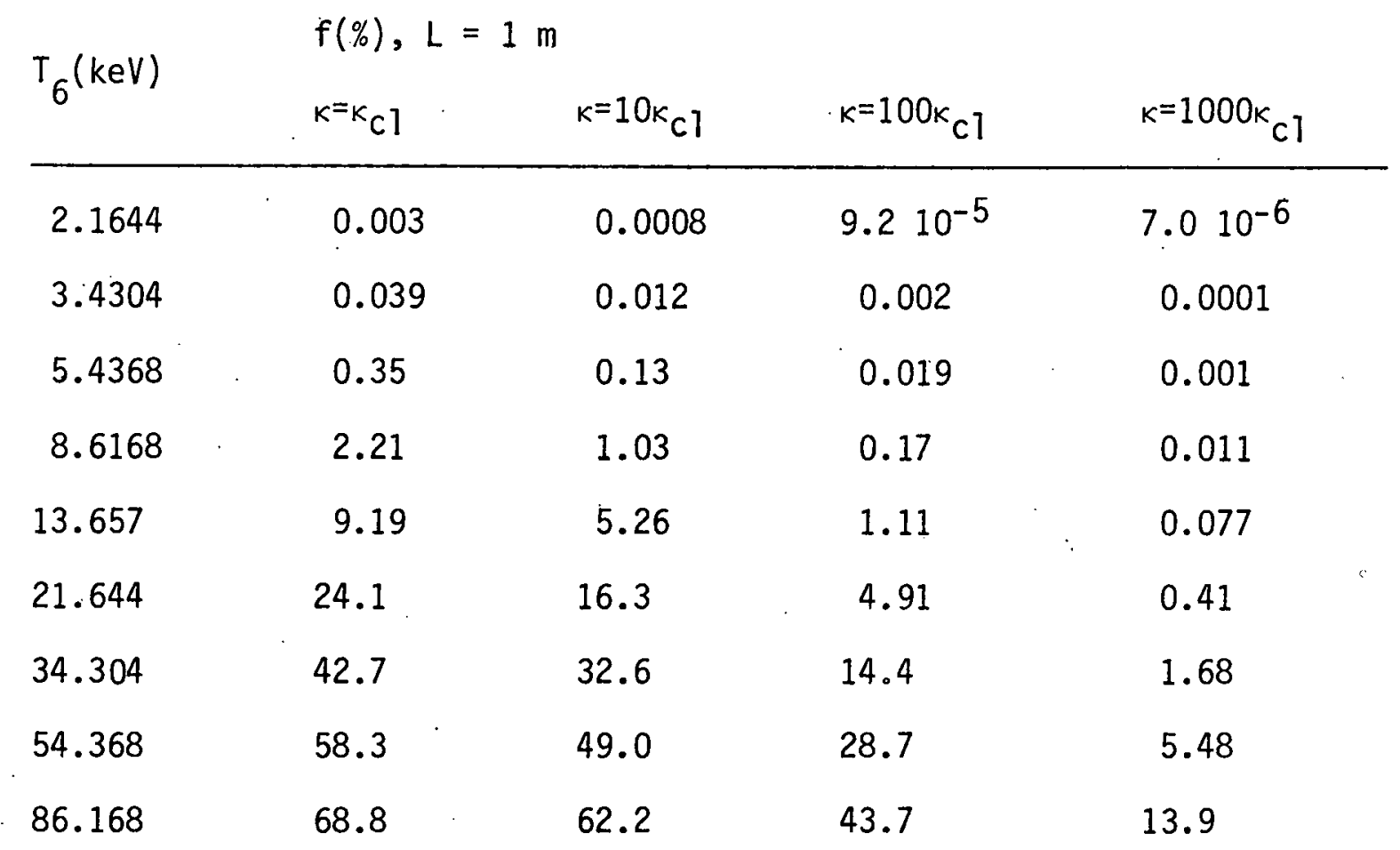

Here the burn fractions, and hence the total energy output, have begun to approach limits, even for sizable thermal loss enhancements. Thus the disadvantage of the tremendous decrease in energy multiplication at reasonable initial temperatures $(T \sim 10-30 \mathrm{keV})$, due to the presence of alpha particle drifts, is offset by the advantage of the decreased sensitivity of most of the central plasma to the enhanced thermal losses - at the wall, allowing the achievement of reasonable, albeit modest, energy 
gains even in the presence of several two-dimensional energy loss mechanisms. These energy gains are very similar to those calculated for the simple radiation-ruled model of equations (3.5).

The results of this chapter show that the behavior of the energy gains and burn fractions calculated from the simple radiation-dominated problem described by equations (3.5) agrees well with the estimates made in chapter 1 from characteristic time arguments. The effect of alpha particle heating is to increase this basic energy gain, slightly in smaller systems and increasingly with growing system size until limiting values are approached, and to cause the energy multiplication to peak at increasing lower initial plasma temperatures for growing system sizes. Enhanced thermal losses due to two-dimensional wal1induced boundary layer instabilities conversely cause the energy multiplication to decrease, greatly for small systems and progressively less as the characteristic length increases, and they cause the location of the peak energy gain to move to higher initial temperatures as the size of the system increases. 


\section{Chapter 4: Discussion and Summary}

The physical phenomena occurring when a hot, dense, deuteriumtritium fusion reacting plasma containing a transverse magnetic field is brought into sudden contact with a cold metal wall have been studied in detail. The results of this computational initial value problem are embodied in the time evolution of the macroscopic plasma properties, such as electron and ion number densities and temperatures, magnetic field and current density, and total pressure. The space- and timedependent energy deposition of the alpha particles produced by fusion reactions was examined.

The energy loss mechanisms in the initially hot plasma are thermal conduction, convection and radiation (bremsstrahlung, line and recombination). These cause the plasma near the wall to cool, with the ion temperature usually being slightly lower than that of the electrons, due to the greater ion transverse thermal conductivity (due to the larger ion gyroradius). The temperatures near the center of the plasma are very dependent on the presence of alpha particle heating. If the initial plasma temperature is too low for significant thermonuclear burn to occur; so that few alpha particles are produced, or if the alpha gyroorbits are not well contained by a strong transverse magnetic field, so that many alpha particles lose all their energy in wall collisions, the central plasma cools by conduction, convection and radiation as does the plasma near the wall, and the total pressure decays with time. For higher initial temperatures or better alpha containment, the central plasma is heated slightly before the energy loss mechanisms dominate, and the total pressure remains almost constant for a significant length 
of time before decaying. When large amounts of alpha particle energy are deposited in the plasma, the central region is dramatically heated, i.e. ignition occurs and a real thermonuclear burn takes place. The radiation losses limit the electron temperature rise, but electron-ion equilibration is often not rapid enough to maintain a single plasma temperature, allowing the ion temperature to be two or three times the radiation-limited electron fluid temperature. The total system pressure rises sharply before the plasma cools and the pressure decays as before.

At the plasma-wall interface, sputtering and evaporation of heavy atoms from the surfaces ir practice can introduce a high flux of impurities into the plasma, which have not been included in the analysis described here. These particles diffuse into the plasma at a rate determined primarily by the charge exchange cross-section, if no major boundary layer instabilities are present. Lehnert [4-1] studied this impurity diffusion problem in the gas blanket case and showed that at fusion-type densities ( $10^{17} \mathrm{~cm}^{-3}$ ) the depth of penetration of these impurities was small for time scales of interest. Vekshtein [4-2] analyzed the rate of impurity diffusion into a wall confined system and concluded from numerical solutions of the diffusion equation that contamination of fusion plasmas was not a serious problem. Jensen [4-3] showed that neutral particles formed at the plasma-wall interface did. not migrate a significant distance into the plasma interior during times of interest to fusion studies; in one characteristic cooling time their concentration was higher than $1 \%$ only within distances a fraction of an ion gyroradius from the wall, while the total heat loss predicted by the full set of boundary layer equations is less than $5 \%$ greater than that of a fully-ionized plasma model with no impurities present. 
The behavior of the energy gains and burn fractions calculated from this one-dimensional plasma-wall problem have been shown to qualitatively agree with simple estimates made from characteristic time arguments; the burn fractions rise sharply with increasing plasma temperature from 1 to $30 \mathrm{keV}$ and then rise very slowly to limiting values, while the energy multiplication rises sharply until the linear rise of the energy input overtakes the rise in burn fraction, creating a peak energy gain for deuterium-tritium around $30 \mathrm{keV}$ followed by a slow decline with rising initial plasma temperature. Varying the system size by an order of magnitude changes the energy gain and burn fraction by a factor of two or three, while changing the initial number density varies the results by less than $50 \%$. The effect of alpha particle confinement and heating is to increase this basic energy multiplication, slightly in smaller systems and increasingly with growing system size. Alpha particle heating also causes the energy gain to peak at lower initial plasma temperatures for growing system sizes. Conversely, enhanced thermal losses due to twodimensional wall-induced boundary layer instabilities, such as those predicted by Lindemuth [4-4], cause the energy multiplication to decrease, drastically for small systems and progressively less as the characteristic dimension increases. They may also cause the location of the peak energy gain to move to higher initial temperatures as the size of the system increases. The parameters for two potential reactor designs (with $Q \sim 10-20$ ) and a smaller "breakeven" experiment (with $Q \sim 2-3$ ) are given in Appendix IV. No alpha particle heating is considered in the data of Appendix IV, since the estimates made in chapter 3 show that the alpha: particles can deposit only a minute fraction of their energy in the plasma before drifting into the chamber walls due to grad $B$ and curvature 
drifts in a cylindrical geometry, for the system sizes considered $(L<1 \mathrm{~m})$. Classical values for ail transport properties are used, since Lindemuth's results show an increase of two or three in thermal conduction lasses in an instability which seems to grow to saturation, rather than continue to increase. The wall loading due to alpha particle collisions ranges from an order of magnitude greater to an order of magnitude less than the pure thermal loading, estimated in chapter 2. The maximum s.ystem pressure in cases studied ranges from about $100 \mathrm{~atm}$ to as high as $1000-2000 \mathrm{~atm}$.

The thermal losses could also be enhanced by the formation of convective cells due to residual motion left from the plasma re-expansion. Here, the plasma sloshing is assumed to occur over only a few Alfven transit times, and the plasma is taken as perfectly uniform and quiescent at the start of the longer burn period. This plasma sloshing could develop magnetic bubbles, complicating and extending the time it would take for magnetic diffusion and plasma convection to bring the plasma to a uniform state. The character of this re-expansion depends greatly. on what is done electrically at the system boundaries. If the dielectric end walls are electrically crowbarred at the start of plasma re-expansion, all the magnetic field is trapped in the cavity, as was assumed in this study. Hence there results a significantly larger imbedded magnetic field ( $\sim 90 \%$ of the piston field) for insulation after the plasma reexpands and fills the cavity, with some further ohmic heating increasing the plasma temperature by $215 \%$. If, at the start of re-expansion, the flux of the magnetic piston is allowed to flow back into the storage. line, partially recharging it, the plasma will expand via a magnetohydrodynamic rarefaction wave, followed by further wave reflections. This 
more controlled, adiabatic re-expansion requires high initial fill magnetic fields (which are now the sole insulation fields) and thus higher power input is needed to compress the plasma to the same final temperature, especially since the plasma temperature drops by a factor of three during the re-expansion. 


\section{Appendix I. Characteristic Times and Lengths}

In all the characteristic lengths and times derived in this section, the electron and ion temperatures and number densities are assumed equal (so that $T_{i}=T_{e}=T$ and $n_{i}=n_{e}=n$ ), and the units, unless otherwise noted, are $[T]=k e V,[n]=m^{-3},[B]=k$ Gauss, $[\ell]=m$ and $[\tau]=\sec$.

The time required for the kinetic energy distribution of a group of particles interacting with themselves to approach a Maxwellian distribution is the self-collision time [I-1]

$$
\tau_{c}=\frac{m^{\frac{3}{2}}(3 k T)^{3 / 2}}{8 \times 0.714 \pi n e^{4} Z^{4} \ln \Lambda}
$$

For a 50/50 mixture of DT the ion self-collision time is therefore

$$
\tau_{c i}=7.11210^{17} \frac{\mathrm{T}^{3 / 2}}{n \ell n \Lambda}
$$

and the electron self-collision time, smaller by the inverse ratio of the thermal velocities, is

$$
\tau_{c e}=1.04310^{16} \frac{T^{3 / 2}}{n \ell n \Lambda}
$$

where the Coulomb logarithm is given by

$$
\begin{aligned}
\Lambda & =\frac{3}{2} \frac{T \mathrm{e}^{3 / 2}}{\mathrm{e}^{3 /} / \frac{\pi n}{\mathrm{~m}}} \\
& =4.89710^{17} \frac{\mathrm{T}^{3 / 2}}{\mathrm{n}^{1 / 2}}
\end{aligned}
$$


The time for equipartition of energy between two groups of particles is $[1-1]$

$$
\tau_{\text {eq }}=\frac{3 m m_{f} k^{3 / 2}}{8(2 \pi)^{\frac{1}{2}} n_{f} z^{2} z_{f}^{2} e^{4} \ln \Lambda}\left(\frac{T}{m}+\frac{T_{f}}{m_{f}}\right)^{3 / 2}
$$

and thus the electron-ion equilibration time (with $m_{e}<m_{i}$ ) is

$$
\tau_{e i}=2.50010^{19} \frac{\mathrm{T}^{3 / 2}}{n \ln \Lambda}
$$

Since $\tau_{e j}=35 \tau_{c i}=2397 \tau_{c e}$, the ordering $\tau_{c e}{ }^{<<\tau_{c i}<<\tau}{ }^{<j}$ holds for all values of number density and temperature.

The ion and electron gyroperiods are

$$
\begin{aligned}
\tau_{g i} & =\frac{2.59410^{-6}}{B} \\
\tau_{g e} & =\frac{5.57810^{-10}}{B}
\end{aligned}
$$

For magnetic fields $\approx 50$ kGauss the characteristic time ordering above becomes

$$
{ }^{\tau} \mathrm{ge}^{<<\tau} \mathrm{gi}^{<<\tau} \mathrm{ce}^{<<\tau} \mathrm{cj}^{<<\tau} \mathrm{ei}
$$

The characteristic slowing down time of an alpha particle on electrons only is [I-2]

$$
\tau_{\alpha}=\frac{3}{8} \frac{m_{\alpha}}{m_{e}} / \sqrt{\frac{m_{e}}{2 \pi}} \frac{T_{e}^{3 / 2}}{\left(e_{1} e_{2}\right)^{2} n_{e} \ell n}
$$




$$
=1.00210^{19} \frac{\mathrm{T}^{3 / 2}}{\mathrm{n} \ln \Lambda}
$$

which is identical to the classical Spitzer alpha-electron equilibration time. The alpha thermalization time is then given by $[1-2]$

$$
\tau_{t h}=\frac{2}{3} \tau_{\alpha} \ln \left[\frac{E_{a}^{3 / 2}+r^{3} T_{e}^{3 / 2}}{E_{t h} 3 / 2+r^{3} T_{e}^{3 / 2}}\right]
$$

where $\quad r^{3}=\frac{3}{4} \frac{\sqrt{\pi}}{m_{i}} \frac{m_{\alpha}^{3 / 2}}{m_{e^{1 / 2}}}=183.46$

At low temperatures (T $1 \mathrm{keV}$ ) where most of the energy loss is to the ions, the alpha thermalization time can be five times greater than the classical alpha-electron equilibration time, while for high temperatures (T $100 \mathrm{keV}$ ) where most of the energy is lost to the electrons, the correct thermalization time is a factor of two less than the electron-only. slowing down time. Thus for most temperatures of interest (T $10 \mathrm{keV}$ )

$$
\tau_{\alpha} \leqslant \tau_{\text {th }} \leqq \tau_{e j}
$$

Defining a radiation cooling time as the average energy stored in the plasma divided by the radiation emission power gives as the characteristic bremsstrahlung loss time

$$
\tau_{b}=4.4810^{20} \frac{T_{!}^{1 / 2}}{n}
$$

where the radiation intensity for a hydrogenic plasma is [I-3] 


$$
\mathrm{P}_{\mathrm{B}}\left(\frac{\mathrm{erg}}{\mathrm{cm}^{3} \mathrm{sec}}\right)=5.3510^{-24} \mathrm{n}^{2}\left(\mathrm{~cm}^{-3}\right) \mathrm{T}^{\frac{1}{2}}(\mathrm{keV})
$$

The $D-T$ reaction time is given by

$$
\tau_{R}=\frac{1}{\frac{1}{2} n \cdot\langle\sigma v\rangle}
$$

where $\sigma$ is the reaction cross section, $v$ is the relative velocity of the $D-T$ particles and the bracket means the average over an ion Maxwellian velocity distribution. Hence $\langle\sigma v>$ is a function of the ion temperature.

A11 the characteristic times above are size-independent, and their ordering is always

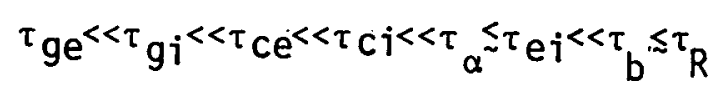

The plasma thermal conduction cooling time is related to the thermal conductivity by $[\mathrm{I}-4]$

$$
\tau_{c}=\frac{n k \ell^{2}}{k}
$$

Using the classical value of perpendicular thermal conductivity, this expression becomes

$$
\tau_{C \perp, i}=3.00810^{20} \frac{T^{\frac{1}{2}} B^{2}}{n \operatorname{lnA}} \ell^{2}
$$




$$
{ }^{\tau_{\perp \perp, e}}=2.05510^{22} \frac{T^{\frac{3}{2}} B^{2}}{n \ell n \Lambda} e^{2}
$$

For comparison, the characteristic cooling time in the absence of an insulating perpendicular magnetic field is

$$
\tau_{c}=2.18210^{-30} \frac{n \ell n \Lambda}{T^{5 / 2}} e^{2}
$$

The drift velocity of an alpha particle in a curved magnetic field is $[1-3]$

$$
v_{D}=\frac{m}{2 q B^{2}}\left(v_{\perp}^{2}+2 v_{11}^{2}\right)(\hat{b} \times \nabla B)
$$

and the corresponding drift time is approximately

$$
\tau_{D}=\frac{\ell}{V_{D}}=1.13610^{-7} \quad \ell^{2} B
$$

where $v_{D} \sim \frac{E_{\alpha}}{q B^{2}} \frac{B}{2 \ell}$.

The characteristic shock heating time can be estimated from the strong shock limits (given in appendix III) as

$$
\begin{aligned}
\tau_{s} & =\frac{L}{u_{1}}+\frac{L / 10}{u_{1} / 2} \\
& =2.21610^{-3} \frac{\ell}{T^{\frac{1}{2}}}
\end{aligned}
$$

where $L=2 l$ is the characteristic size of the system. 
The Debye length is given by [I-1]

$$
\begin{aligned}
\lambda_{D} & =\sqrt{\frac{k T_{e}}{4 \pi n_{e} e^{2}}} \\
& =2.35110^{5} \frac{T^{\frac{3}{2}}}{n^{\frac{1}{2}}}
\end{aligned}
$$

and the self-collision length (mean free path) is

$$
\ell_{c}=\frac{\left(3 k T_{e}\right)^{2}}{8 \times 0.714 \pi n e_{e} e^{4} \ell n}
$$

For both electrons and DT ions this becomes

$$
\ell_{c}=2.41910^{23} \frac{T^{2}}{n \ell m}
$$

The ion gyroradius is

$$
r_{g i}=\frac{m v_{\perp}}{e B}=8.15310^{-2} \frac{T^{\frac{1}{2}}}{B}
$$

The frequency-dependent mean free path for radiation absorption is $[\mathrm{I}-5]$

$$
\lambda_{p}^{\nu}(\mathrm{cm})=710^{-5} \frac{\mathrm{T}^{\frac{1}{2}}(\mathrm{keV})}{\mathrm{n}_{\mathrm{e}}^{2}\left(\mathrm{~cm}^{-3}\right)} v^{3}\left(\mathrm{sec}^{-1}\right)
$$

The power emitted per unit wavelength per unit volume due to ionelectron bremsstrahlung is 


$$
\begin{aligned}
& \frac{\mathrm{dP}}{d \lambda}\left(\frac{\mathrm{erg}}{\mathrm{cm}^{3} \AA \mathrm{sec}}\right)= \\
& 6.0110^{-23} \mathrm{n}_{\mathrm{e}}\left(\mathrm{cm}^{-3}\right) \mathrm{n}_{i}\left(\mathrm{~cm}^{-3}\right) \mathrm{T}_{\mathrm{e}}^{\frac{1}{2}}(\mathrm{keV}) \lambda^{-2}(\stackrel{\circ}{\AA}) \exp -\left[\frac{12.4}{\lambda_{\lambda}(\AA) \mathrm{T}_{\mathrm{e}}(\mathrm{keV})}\right]
\end{aligned}
$$

which has a sharp maximum at

$$
\lambda_{\max }(\stackrel{\circ}{A})=\frac{6.2}{T_{e}(k e V)}
$$

The frequency dependent mean free path corresponding to this maximum wavelength is then

$$
\lambda_{p}^{\max }=7.93010^{58} \frac{T^{7 / 2}}{n^{2}}
$$

which is enormous compared to any of the other characteristic lengths. The mean free path for neutrons undergoing oniy nuclear force interactions with the plasma ions (reactions with cross sections on the order of a barn) is

$$
l_{n}=\frac{1}{n_{\sigma}}=10^{28} \frac{1}{n}
$$

These characteristic lengths can be ordered to give

$$
\lambda_{D}<<r_{g i}<<l<l_{C}<<l_{n}<<\lambda_{p}^{\max }
$$

The alpha gyroradius at $3.5 \mathrm{MeV}$ is 


$$
\begin{aligned}
r_{\alpha} & =\frac{m_{\alpha} v}{e B} \\
& =5.405 \frac{1}{B}
\end{aligned}
$$

which fits into the overall length ordering through $r_{g i} \leq r_{\alpha} \leq \ell$. 
Appendix II. Alpha Particle Modelling

Alpha particles, which are born at $3.5 \mathrm{MeV}$ in a fusion grade O-T plasma and which transfer their kinetic energy to the background plasma, represent a time-and space-dependent plasma heating source.

\section{A. Time Dependence}

Since there is a high energy source term in the alpha particle distribution function and since the time steps used during the computation are much smaller than a characteristic thermalization time, the alpha particles are not described by a Maxwellian distribution but are instead treated as test particles, while the background plasma distribution functions are assumed to be Maxwellian.

The rate of change of the energy of a test particle due to interactions with a Maxwellian background of field particles can be derived from the Fokker-Planck equation for a delta function test particle distribution [II-1, II-2] and from binary col]ision theory [II-3]. For the electron fluid because of the large mass ratio, the alpha particle velocity can aiways be assumed much smaller than the electron mean thermal velocity, and the energy loss from the $\alpha$ particles to the electron fluid can be reduced to

$$
\left.\frac{d E_{\alpha}}{d t}\right)_{e}=-\frac{E_{\alpha}}{\tau_{\alpha}}=-\frac{8}{3} \frac{\sqrt{2 \pi}}{T_{e}^{3 / 2}} n_{e} \frac{\sqrt{m_{e}}}{m_{\alpha}^{*}}\left(e_{\alpha}^{2} e_{e}^{2}\right) \ln \Lambda E_{\alpha}
$$

where the coefficient $\tau_{\alpha}$ is identical to the classical Spitzer electronalpha equilibration time. If (as is usually done for equal electron and 
ion temperatures and densities in the plasma) the alpha particle speed is assumed much greater than the average ion speed, the rate of energy lost to the ion fluid is

$$
\left.\frac{d E_{\alpha}}{d t}\right)_{i}=-\frac{4 \pi}{\sqrt{2}} n_{i} \frac{\sqrt{m_{\alpha}}}{m_{i}}\left(e_{\alpha}^{2} e_{i}^{2}\right) \ln \Lambda \frac{1}{\sqrt{E_{\alpha}}}
$$

al though to correctly model the thermalization process itself (where $E_{\alpha} \geq k T_{i}$ so that $v_{\alpha} \sim v_{t h_{j}}$ ) this approximate equation must be replaced by the accurate expression

$$
\begin{gathered}
\left.\frac{d E_{\alpha}}{d t}\right)_{i}=-\frac{4 \pi}{\sqrt{2}} n_{i} \frac{\sqrt{m_{\alpha}}}{m_{i}}\left(e_{\alpha}{ }^{2} e_{j}{ }^{2}\right) \ln \Lambda \frac{1}{\sqrt{E_{\alpha}}} \operatorname{erf}\left(\frac{v_{\alpha}}{v_{t h_{j}}}\right) . \\
+\frac{8 \sqrt{\pi}}{v_{t h_{i}}} n_{i} \frac{\left(m_{j}+m_{\alpha}\right)}{m_{i}^{m_{\alpha}}}\left(e_{\alpha}{ }^{2} e_{i}{ }^{2}\right) \quad \ln \Lambda \exp \left(-\frac{v_{\alpha}{ }^{2}}{v_{t h_{j}}{ }^{2}}\right)
\end{gathered}
$$

When $v_{\alpha} \gg v_{t h}$, this expression does indeed reduce to equation AII.2. In the derivation of these expressions, collective effects were incorporated only through Debye shielding in the Coulomb cross-section. Comparisons of results obtained using these equations with those of Sigmar and Joyce [II-4] in which plasma collective effects are accounted for more exactly through the plasma dielectric constant in the BalescuLenard equations, show good quantitative agreement [II-5, II-6]. The classical cutoff angle which is strictly valid only in the limit of very small relative velocities is used throughout, al though an energetic alpha particle can slow down from a region where the quantum cutoff angle is valid, through a region where neither quantum nor classical 
values apply, into a region where the classical result is generally valid. Calculations [II-3] which incorporate both the classical and quantum cutoffs and an approximate transition between the two show that results from these two limits differ by ten to twenty percent.

To study the energy deposition of an arbitrary distribution of alpha particles requires integration of the single particle slowing down equations over the alpha energy spectrum, and can be approximated by discrete summation over alpha particle groups.

If $\delta n_{\alpha}{ }^{k}$ is defined as the number of alpha particles born at any time step $t_{k}$ and $E_{\alpha}{ }^{k}$ is defined as the energy to which an alpha particle born at time $t_{k}$ will have slowed down at the current time $t_{j}$ (where $k \leqq j$ ), the alpha heating terms in the ion and electron temperature equations $(2.30)$ and $(2.35)$ are given by

$$
Q_{\alpha i, e}=\sum_{k=\ell+1}^{j} \delta n_{\alpha}{ }^{k} \Delta E_{\alpha i, e}^{k}
$$

Where all alpha particles not yet thermalized to the ion fluid temperature $\left(k>\ell\right.$, where $\left.E_{\alpha}^{\ell} \leq 0<E_{\alpha}^{\ell+1}\right)$ contribute various amounts of energy calculated from the single particle equations (AII.1), and (AII.2) or (AII.3), as

$$
\left.\Delta E_{\alpha j, e}^{k}=\frac{d E_{\alpha} k}{d t}\right)_{j, e} \Delta t
$$

Since the ion fluid contains thermalized $(k \leq \ell)$ alpha particles,

$$
n_{j} \equiv n_{D}+n_{T}+\sum_{k=1}^{\&} \delta n_{\alpha} k
$$


The change in the alpha particle distribution at a time step $t_{j}$ is given by

$$
\delta n_{\alpha}{ }^{k}\left(t_{j+1}\right)= \begin{cases}\frac{\partial n_{\alpha}}{\partial t} \Delta t & k=j+1 \\ \delta n_{\alpha} k\left(t_{j}\right) & k \leq j\end{cases}
$$

and

$$
E_{\alpha}{ }^{k}\left(t_{j+1}\right)= \begin{cases}3.52 \mathrm{MeV} & k=j+1 \\ E_{\alpha}{ }^{k}\left(t_{j}\right)-\left(\Delta E_{\alpha i}{ }^{k}+\Delta E_{\alpha e}{ }^{k}\right) & \ell<k \leq j \\ 0 & 1 \leq k \leq \ell\end{cases}
$$

This method demands both storage of information from, and summation over all previous time steps at each time step and can be very costly in computer time. The integration of the single particle equations is instead approximated by a discrete summation over a small number of fixed alpha particle energy groups.

If $E_{\alpha}{ }^{k}$ is defined as $N$ logarithmically spaced fixed alpha particle energies and $\delta n_{\alpha}{ }^{k}$ is defined as the number of alpha particles of energy $E_{\alpha}{ }^{k}$ at the current time, the alpha heating of ions and electrons is given by

$$
Q_{\alpha i, e}=\sum_{k=1}^{N} \delta n_{\alpha}{ }^{k} \Delta E_{\alpha i, e}^{k}
$$


where as before all non-thermalized alpha particles $(1 \leq k \leq N$, since $E_{\alpha}{ }^{k}>0$ ) contribute various amounts of energy calculated from the single particle equations, as in equation (AII.5). The ion fluid contains thermalized alpha particles, as in equation (AII.6).

The energy group populations $\delta n_{\alpha}^{k}$ are updated at the end of each time step by

(1) calculating the new energy $E_{\alpha}{ }^{\prime}$ to which alpha particles of energy have slowed down in a time $\Delta t$, given by

$$
E_{\alpha}^{k^{\prime}}=E_{\alpha}^{k}-\left(\Delta E_{\alpha j}^{k}+\Delta E_{\alpha e}^{k}\right)
$$

(2) splitting these alpha particles between the two bracketing groups $E_{\alpha}{ }^{m-1}<E_{\alpha}{ }^{k^{\prime}}<E_{\alpha}{ }^{m}$ (or between the ion fluid and the lowest non-thermal group $E_{\alpha}^{k^{\prime}} \leq 0<E_{\alpha}^{\prime}$ ) using particle and energy conservation to give

$$
\begin{gathered}
n_{\alpha}{ }^{k}\left(t_{j}\right) E_{\alpha}{ }^{\prime}=n_{\alpha}^{m-1}\left(t_{j+1}\right) E_{\alpha}^{m-1}+n_{\alpha}^{m}\left(t_{j+1}\right) E_{\alpha}^{m} \\
n_{\alpha}{ }^{k}\left(t_{j}\right)=n_{\alpha}^{m-1}\left(t_{j+1}\right)+n_{\alpha}{ }^{m}\left(t_{j+1}\right)
\end{gathered}
$$

(3) and, after advancing all $\mathrm{N}$ groups as described above, adding newly produced alpha particles $\frac{\partial n_{\alpha}}{\partial t} \Delta t$ to the source $\left(E_{\alpha}{ }^{N}=3.52 \mathrm{MeV}\right)$ group.

The relative accuracy and expense of these two discrete sums, and their comparison to models assuming Maxwellian distributions of aipha particles, can be studied using a simple zero-dimensional two fluid plasma wt th bremsstrahlung cooling and alpha particle heating. 
described by

$$
\begin{aligned}
& \left.\frac{\partial n_{\alpha}}{\partial t}=-\frac{\partial n_{D}}{\partial t}=-\frac{\partial n_{T}}{\partial t}=n_{D} n_{T}<\sigma V\right\rangle \\
& \frac{\partial T_{i}}{\partial t}=410^{-20} n_{e} \ln \Lambda \frac{T_{e}-T_{i}}{T_{e}^{3 / 2}}+Q_{\alpha i} \\
& \frac{\partial T_{e}}{\partial t}=410^{-20} n_{i} \ln \Lambda \frac{T_{j}-T_{e}}{T_{e}^{3 / 2}}+Q_{\alpha_{e}}-4.4610^{-21}\left(n_{D}+2 n_{\alpha}\right) T_{e}^{\frac{1}{2}}
\end{aligned}
$$

Beside the discrete sums in equations (AII.4) and (AII.7), the alpha heating terms $Q_{\alpha j}$ and $Q_{\alpha e}$ can be given by [II-7]

$$
\begin{aligned}
& Q_{\alpha i}=\frac{2}{3 n_{i}} \quad f E_{\alpha} \frac{\partial n_{\alpha}}{\partial t} \\
& Q_{\alpha}=\frac{2}{3 n_{e}} \quad(1-f) E_{\alpha} \frac{\partial n_{\alpha}}{\partial t}
\end{aligned}
$$

where the alpha particles are assumed to immediately become part of the ion Maxwellian fluid (so that $n_{i} \equiv n_{D}+n_{T}+n_{\alpha}$ ) and their excess energy $E_{\alpha}$ is divided between the electron and ion fluids, the fraction going to the latter being given by

$$
f=\frac{32}{32+T_{e}}
$$

If the alpha particles are asșumed to form a third, Maxwellian fluid of temperature $T_{\alpha}[I I-8]$, the heating terms are given by 


$$
Q_{\alpha i, e}=\frac{T_{\alpha}-T_{j, e}}{\tau_{\alpha i, e}}
$$

where the equilibration times are the classical Spitzer expressions

$$
\begin{aligned}
\tau_{\alpha i} & =1.83310^{16} \frac{\left(2.5 \mathrm{~T}_{\alpha}+4 \mathrm{~T}_{j}\right)^{3 / 2}}{n_{\alpha} \ell n \Lambda} \\
\tau_{\alpha P} & =1.010^{19} \frac{T_{C}{ }^{3 / 2}}{n_{\alpha} \ell n \Lambda}
\end{aligned}
$$

and where the alpha fluid temperature is given by the equation

$$
\frac{\partial T_{\alpha}}{\partial t}=\frac{\left(2333-T_{\alpha}\right)}{n_{\alpha}} \frac{\partial n_{\alpha}}{\partial t}-\frac{n_{i}}{n_{\alpha}} Q_{\alpha i}-\frac{n_{e}}{n_{\alpha}} Q_{\alpha e}
$$

in which the value of $2333 \mathrm{KeV}$ comes from $3.52 \mathrm{MeV}=\frac{3}{2} \mathrm{kT} \mathrm{\alpha}_{\alpha_{0}}$. The ion fluid in this case contains no alpha particles, so $n_{j} \equiv n_{D}+n_{T}$ only.

The electron and ion temperatures given by equations (AII.8) and (AII.9) are shown in figures 1 and 2, with alpha heating given by the four expressions (AII.4), (AII.7), (AII.10) and (AII.11). The discrete sum of all previous time step data is the benchmark calculation, to which the other three approximations are compared.

All three models overestimate the peak electron temperature almost equally. However, the instantaneous deposition and three fluid models give a slower temperature rise and hence a later in time peak than the energy group model, whose shape agrees much better with the benchmark calculation.

The more important (because it governs reaction rate) ion temperature is drastically overestimated by the instantaneous deposition 


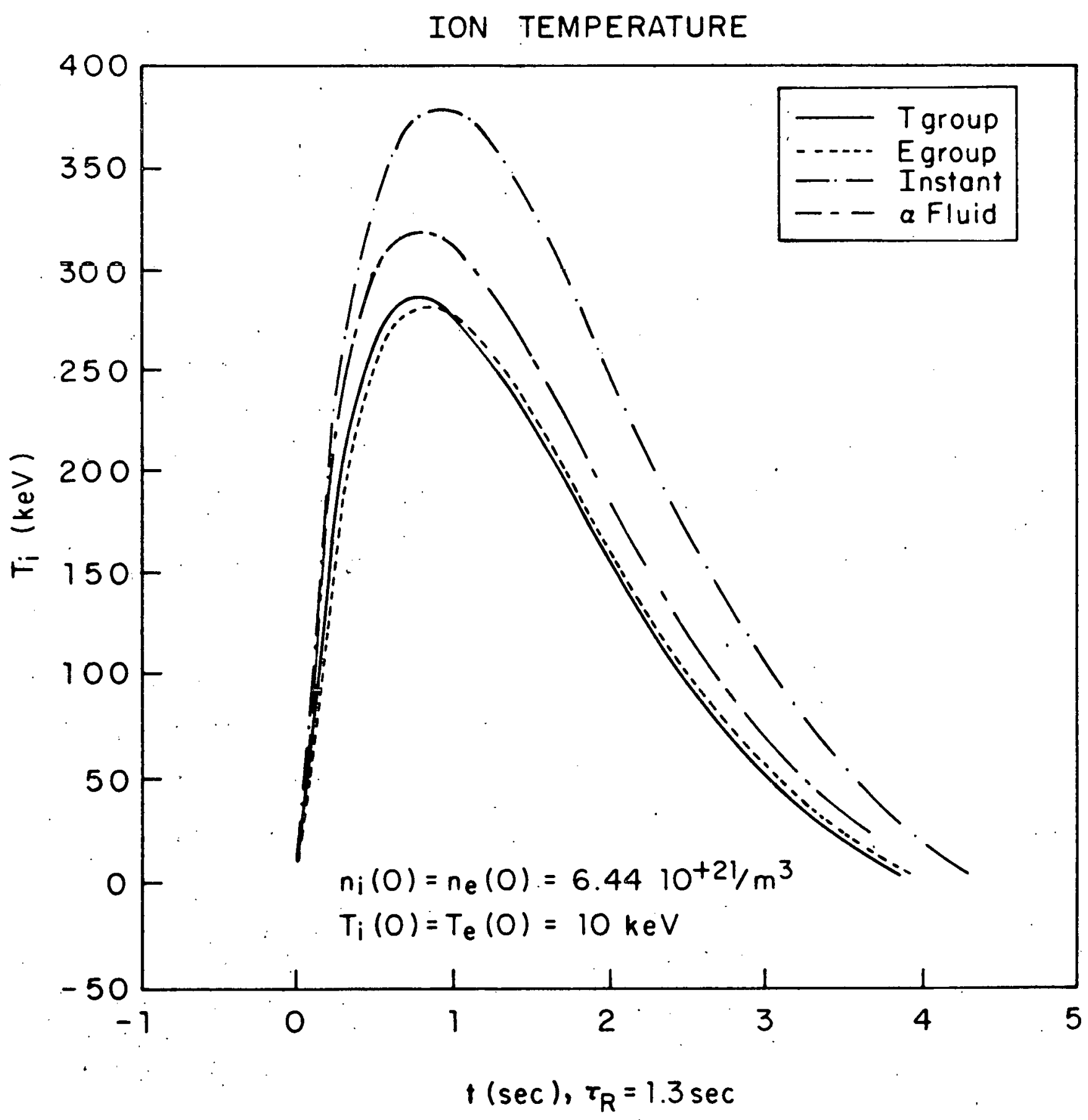

Figure 1 (appendix II) Time evolution of the ion temperature for different models of the time dependence of alpha particle energy deposition 


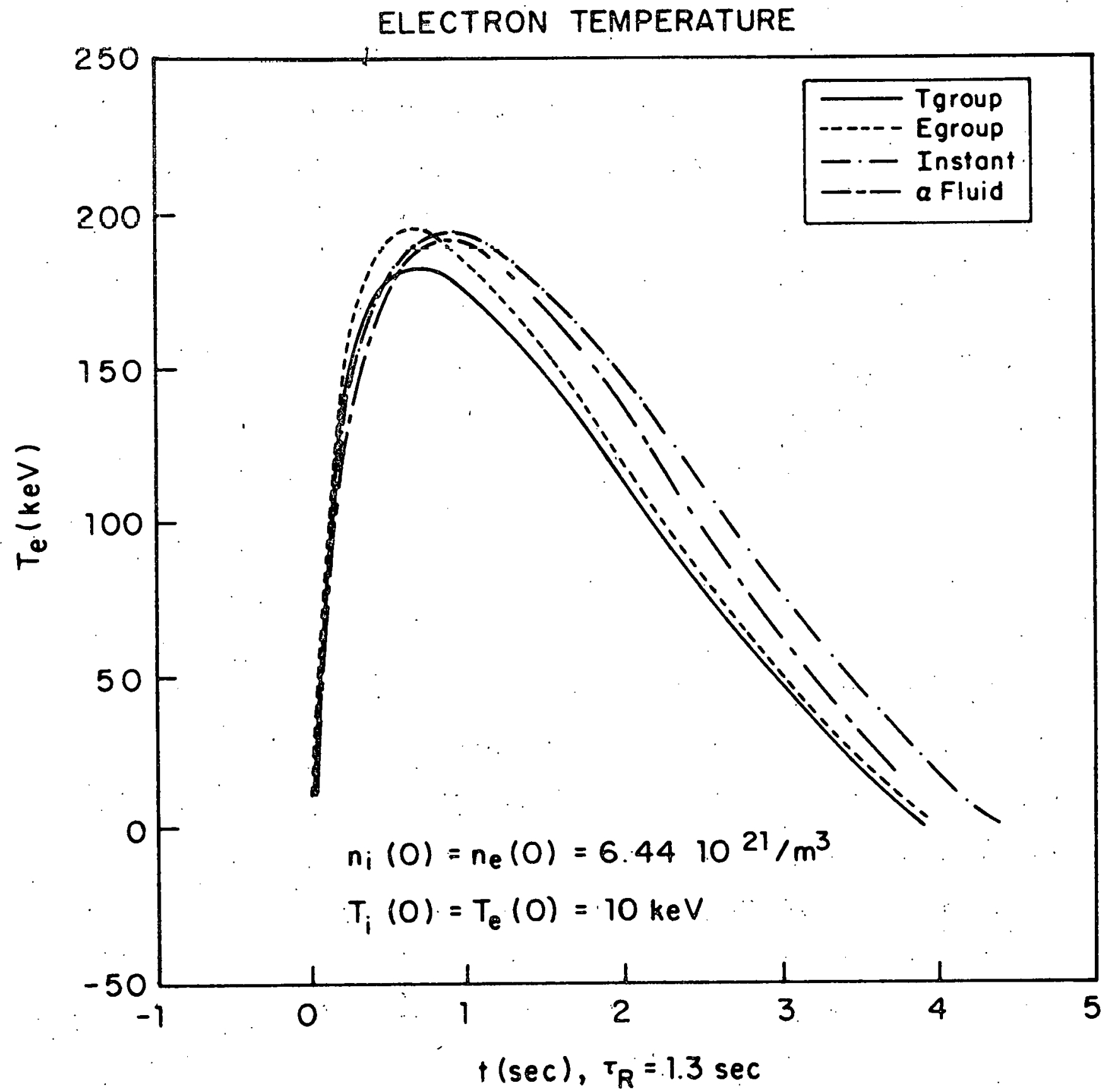

Figure 2 (appendix II) Time evolution of the electron temperature for different models of the time dependence of alpha particle energy deposition 
model which dumps too much heat too quickly, and still greatly overestimated by the three fluid model, while the energy group result is slightly conservative.

While run times for the energy group model are two to three times greater than those for the other two (simpler) models, they are still at least a factor of ten less than those for the benchmark study. This approach thus combines reasonable accuracy with computer efficiency.

\section{B. Spatial Dependence}

The maximum radius of gyration of an alpha particle in the presence of a magnetic field is given by

$$
r_{\alpha}=\frac{m_{\alpha} v}{q_{\alpha} B}=0.0911 \frac{\sqrt{E_{\alpha}(k e V)}}{B(k G)}
$$

where $\frac{1}{2} \mathrm{mv}^{2}=\frac{3}{2} \mathrm{kT}$. For a $3.52 \mathrm{MeV}$ alpha particle this ranges from $10.8 \mathrm{~cm}$ at $50 \mathrm{kG}$. to $3.6 \mathrm{~cm}$ at $150 \mathrm{kG}$, while for a $100 \mathrm{keV}$ alpha particle the radius varies from $1.8 \mathrm{~cm}$ at $50 \mathrm{kG}$ to $0.6 \mathrm{~cm}$ at $150 \mathrm{kG}$. Since some of the alpha.particle kinetic energy lies in velocity parallel to the magnetic field, the actual gyroradius is usually less than the above values. In problems where the spatial cells are on the order of a few centimeters or less, the alpha particle orbits can cover significant background gradients of temperature and number density, whose effect cannot be neglected by assuming pure local deposition. In the presence of a wall the orbits can also intersect a material barrier and thus the alpha particle energy can be lost from the plasma. 
The spatial dependence of the alpha particle energy deposition can be modelled by assuming that the energy loss in any given cell traversed is direcliy proportional to the fractional time spent in the cell, and that the time spent in any cell is directly proportional to the fractional track length contained in the cell, calculated from the gyroradius distribution function.

For an alpha particle emitted isotropically (a good assumption at non-relativistic energies) in spherical coordinates with the principal axis $z$ parallel to the magnetic field, the angle $\phi$ will be uniformly distributed between 0 and $\pm \pi$ so that

$$
\phi=\pi(2 w-1)
$$

where $w$ is a random variable uniformly distributed between 0 and 1 . The distribution in $\theta$ for a $2 \pi$. half-sphere is given by

$$
\theta=\cos ^{-1} w
$$

Since the perpendicular velocity is given by $v \cdot \sin \theta$, the probability density function for the alpha particle radius of gyration is distributed according to $\sin \theta$, and properly normalized is given by

$$
f(w, \phi)=\frac{\sqrt{1-w^{2}}}{\int_{0}^{1} \int_{0}^{2 \pi} \sqrt{1-w^{2}} d w d \phi}=\frac{2}{\pi^{2}} \sqrt{1-w^{2}}
$$


since the gyroradius is given by

$$
\begin{aligned}
r_{\alpha} & =\frac{m_{\alpha} v}{q_{\alpha} B}=\frac{m_{\alpha} v \sin \theta}{q_{\alpha} B} \\
& =r_{\max } \sin \theta=r_{\max } \sqrt{1-w^{2}}
\end{aligned}
$$

The total a.lpha particle track length, neglecting possible wall collisions, is

$$
\begin{aligned}
\ell & =\int_{0}^{1} \int_{0}^{2 \pi} 2 \pi r_{\alpha}(w, \phi) f(w, \phi) d w d \phi \\
& =\int_{0}^{1} \int_{0}^{2 \pi} 2 \pi r_{\max } \sqrt{1-w^{2}}\left(\frac{2}{\pi^{2}} \sqrt{1-w^{2}}\right) d w d \phi \\
& =\frac{16}{3} r_{\max }
\end{aligned}
$$

which is less than. $2 \pi r_{\max }$, as expected.

Given a cell structure as described in chapter 2 , with cell boundaries $x_{j}$ and cell centers $\bar{x}_{j}$, with walls at $x_{0} \equiv 0$ and $x_{j+1}=L$, the track length of an alpha particle born at cell center $\bar{X}_{j}$ that lies in cel1 $i$ is given by

$$
\ell_{i j}=\iint 2 r_{\alpha}(w, \phi) \psi_{i j}(w, \phi) f(w, \phi) d w d \phi
$$

where $2 r_{\alpha}(w, \phi) \psi_{i j}(w, \phi)$ is the arc length, lying between $x_{i-1}$ and $x_{j}$, of a circle with center coordinate defined by 


$$
x^{*}=\bar{x}_{j}+r_{\max } \sqrt{1-w^{2}} \sin \phi
$$

(Since $y$. is an ignorable coordinate, the circle centers can all be assumed to $7 i$ e on the same horizontal line.) For $i \neq j$, the track length is

$$
\begin{aligned}
\ell_{i j} & =\mid \int_{1}^{w_{1}} \int \frac{4 r_{\max }}{\pi^{2}}\left(1-w^{2}\right) \cos ^{-1}\left(2 \sqrt{\frac{1-w_{2}^{2}}{1-w^{2}}}+\sin \phi\right) d w d \phi \\
& -\int_{1}^{w_{3}} \int_{1}^{\phi_{3}} \frac{4 r_{\max }}{\pi^{2}}\left(1-w^{2}\right) \cos ^{-1}\left(2 \sqrt{\frac{1-w_{3}^{2}}{1-w^{2}}}+\sin \phi\right) d w d \phi \mid
\end{aligned}
$$

where we define

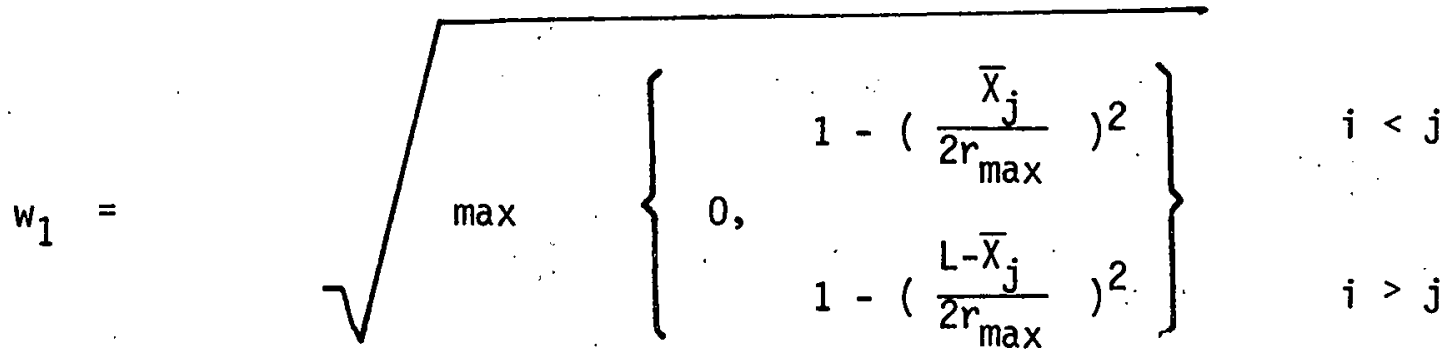

$w_{2}=\sqrt{\max \left\{\begin{array}{cc}0, & 1-\left(\frac{\bar{x}_{j}-x_{i}}{2 r_{\max }}\right)^{2} \\ 0, & 1-\left(\frac{\bar{x}_{j}-x_{j-1}}{2 r_{\max }}\right)^{2}\end{array}\right\}} \quad \begin{aligned} & i<j \\ & i>j\end{aligned}$ 


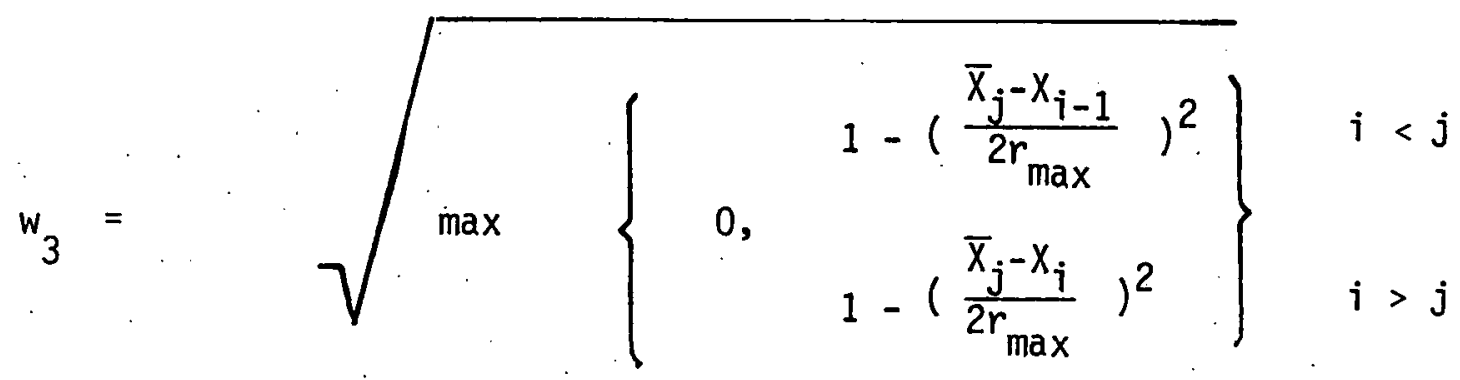

and

$\phi_{1}(w)= \begin{cases}\sin ^{-1}\left[\max \left(-1,1-\frac{\bar{x}_{j}}{r(w)}\right)\right] & i<j \\ \sin ^{-1}\left[\min \left(1,-1+\frac{L-\bar{x}_{j}}{r(w)}\right)\right] & i>j\end{cases}$

$\phi_{2}(w)= \begin{cases}\sin ^{-1}\left[\max \left(-1,1+\frac{x_{j}-\bar{x}_{j}}{r(w)}\right)\right] & i<j \\ \sin ^{-1}\left[\min \left(1,-1+\frac{x_{i-1}-\bar{x}_{j}}{r(w)}\right)\right] & i>j\end{cases}$

$\phi_{3}(w)= \begin{cases}\sin ^{-1}\left[\max \left(-1,1+\frac{x_{i-1}-\bar{x}_{j}}{r(w)}\right)\right] & i<j \\ \sin ^{-1}\left[\min \left(1,-1+\frac{x_{i}-\bar{x}_{j}}{r(w)}\right)\right] & i>j\end{cases}$

The contribution to the origin cell $(i=j)$ is easily found by subtraction, and is 
$\ell_{j j}=\frac{16}{3} r_{\max }-2 \int_{0}^{w_{2}(i<j)} \int_{-\frac{\pi}{2}}^{\phi_{2}(i<j)} \frac{4 r_{\max }}{\pi^{2}}\left(1-w^{2}\right) \cos ^{-1}\left(2 \sqrt{\frac{1-w_{2}^{2}}{1-w^{2}}}+\sin \phi\right) d w d \phi$

The track length intersecting the wall can be found in two separate ways, and is given by

$$
\begin{aligned}
& e_{j}^{w a l l}=\int_{0}^{w_{1}} \int_{-\frac{\pi}{2}}^{\frac{\pi}{2} i<j} \frac{4 r_{\max }}{\phi^{2}}\left(1-w^{2}\right) \cos ^{-1}\left(2 \sqrt{\frac{1-w_{1}^{2}}{1-w^{2}}}+\sin \phi\right) d w d \phi \\
& \phi_{1} \quad i>j \\
& =\frac{16}{3} r_{\max }-\sum_{i=1}^{20} \ell_{i j}
\end{aligned}
$$

The fractional track length lying in each cell is then

$$
s_{i j}=\frac{\ell_{i j}}{\sum_{i=1}^{20} \ell_{i j}}
$$

and the fraction of alpha particles lost to wall collisions is

$$
s_{j}{ }^{\text {wall }}=1-\frac{\sum_{i=1}^{20} e_{i j}}{\frac{16}{3} r_{\max }}
$$

These integrals have been evaluated numerically using an adaptive Simpson scheme, and require about one minute of computer time for a problem with twenty spacial cells and ten discrete alpha energy groups. A faster and simpler method for the same problem is to use 
Monte Carlo techniques, in which the essentially trigonometric nature of this model is very evident.

For any given source cell with center $\bar{x}_{j}$, and alpha particle energy $E_{\alpha}$ determining $r_{\max }$ (which, for the discrete energy groups described in part $A$, requires a third subscript determining $E_{\alpha}^{k}$ and $\left.r_{\max }^{k}\right)$, the procedure is given below.

(1) The particle's initial direction is chosen randomly from an isotropic distribution $(w=\cos \theta, \phi)$, determining the orbit center

$$
x^{*}=\bar{x}_{j}+r_{\max } \sqrt{1-w^{2}} \sin \phi
$$

(2) If the orbit intersects either wall $\left(X^{*}-r \leq 0\right.$ or $\left.X^{*}+r \geq L\right)$, the particle is binned in the wall-collision tally and a new particle is started (fig. 3a).

(3) If the orbit is entirely contained within the walls, the bracketing cells $i_{1}$ and $i_{2}$ are determined and the fractional arc lengths which are added to the track length tallies are (fig. 3b)

$$
s_{i j}= \begin{cases}0 & i<i_{1} \\ \frac{1}{\pi} \cos ^{-1}\left(\frac{x^{*}-x_{i 1}}{r}\right) & i=i_{1} \\ \frac{1}{\pi}\left[\cos ^{-1}\left(\frac{x^{*}-x_{i}}{r}\right)-\cos ^{-1}\left(\frac{x^{*}-x_{i-1}}{r}\right)\right] & i_{1}<i<i_{2} \text { (AII.18) } \\ \frac{1}{\pi}\left[\pi-\cos ^{-1}\left(\frac{x^{*}-x_{i 2-1}}{r}\right)\right] & i=i_{2} \\ 0 & i>i_{2}\end{cases}
$$


(3a)
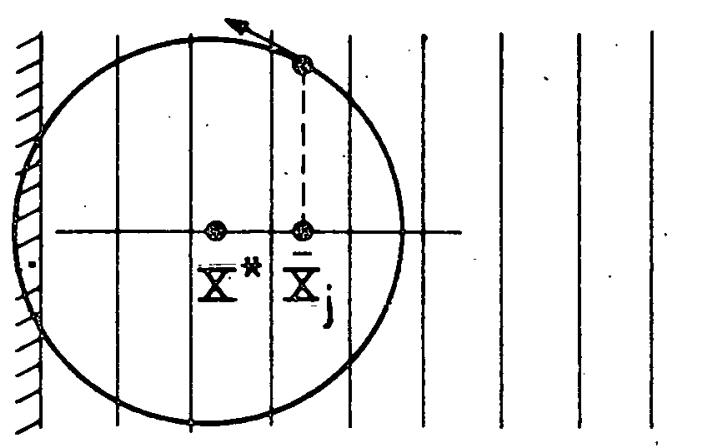

(3b)
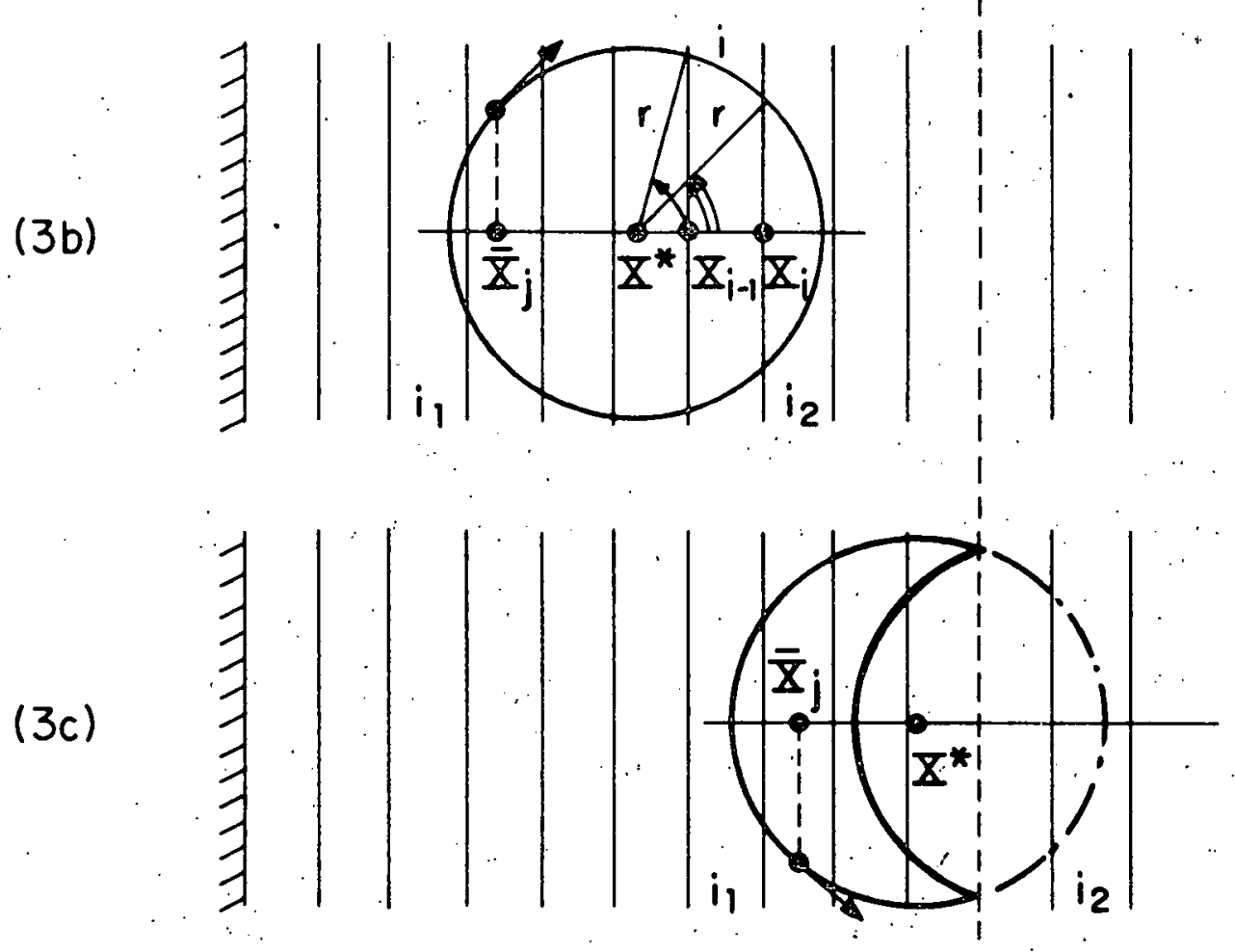

Figure 3 (appendix II) Alpha particle orbits which (a) intersect the wal1, (b) are perfectly contained, or (c) are contained but pass through the symmetry plane 
(4) If the orbit passes through the symmetry plane, it is reflected (fig. $3 c$ ) so that

$$
s_{i j}=\left\{\begin{array}{lc}
s_{i j}+s_{21-i, j} & 1 \leq i \leq 10 \\
0 & 11 \leq i \leq 20
\end{array}\right.
$$

(5) When as many particles as are needed to give the required statistical accuracy $\left(\frac{1}{\sqrt{N}}\right)$ have been tallied, the fractional track lengths are normalized by the total number of particles that did not intersect the wall, while the wall collision tally is normalized by the total number of particles started in step (1).

While numerical integration required about one minute of computer time for a problem with twenty spacial cells and ten discrete energy groups, the Monte Carlo techniques detailed above require about one second to run 500 particles per source cell per energy group.

The fractional track lengths are shown in figures 4 and 5 for two different source cells and for ten logarithmically spaced energy groups ranging from $1 \mathrm{keV}$ to $3.52 \mathrm{MeV}$. The effect of reflection through the symmetry plane can be seen in figure 5 , especially for $k=10$.

The alpha heating terms in the ion and electron temperature equations (2.30) and (2:35), described in part $A$, are modified to become

$$
Q_{\alpha i, e}^{j}=\sum_{k=1}^{10} \delta n_{\alpha}^{j k} \Delta E_{\alpha i, e}^{j k}
$$




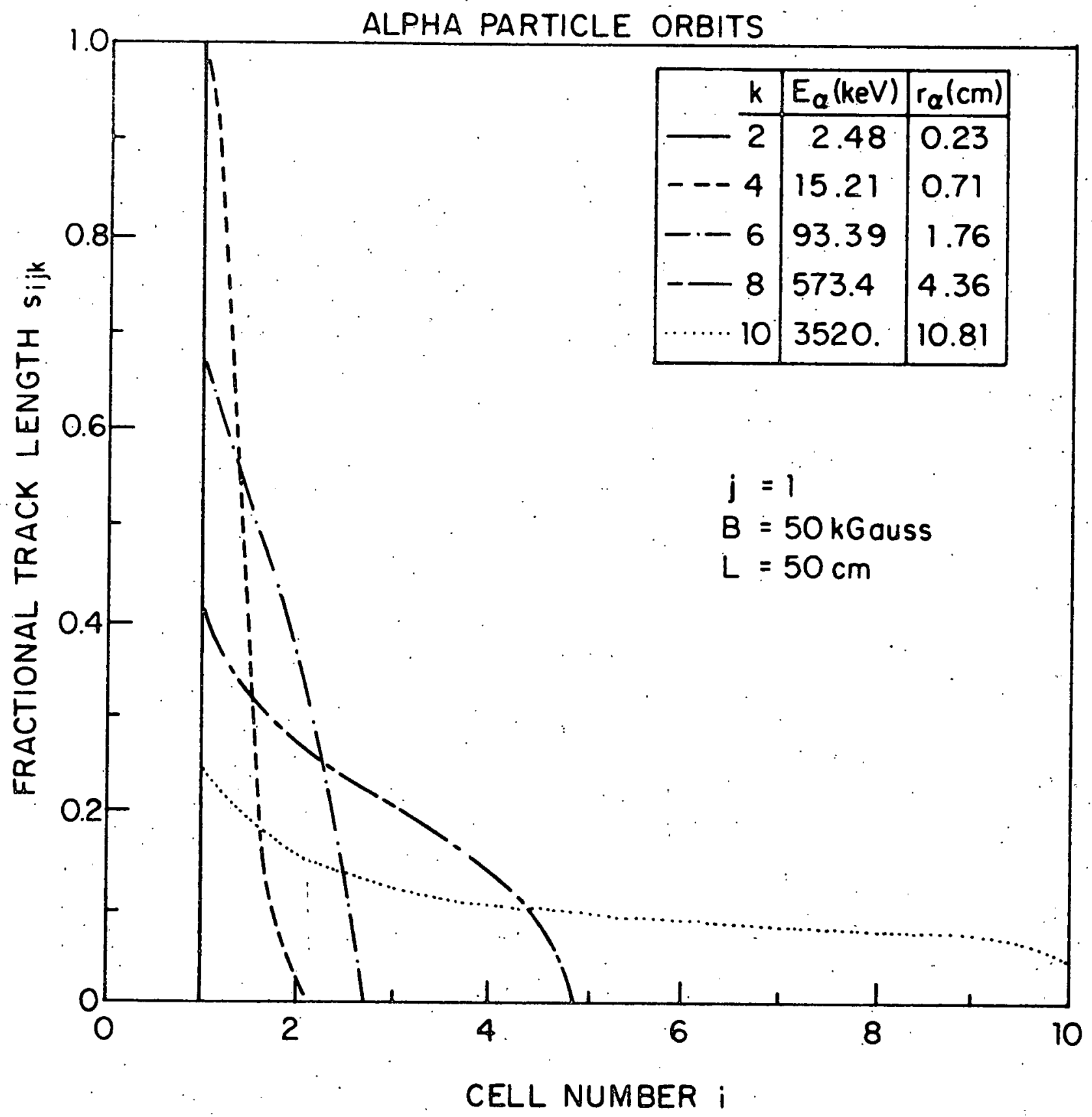

Figure 4 (appendix II) Fractional track length of an alpha particle born in cell $j$ which lies in cell $i$ 
ALPHA PARTICLE ORBITS

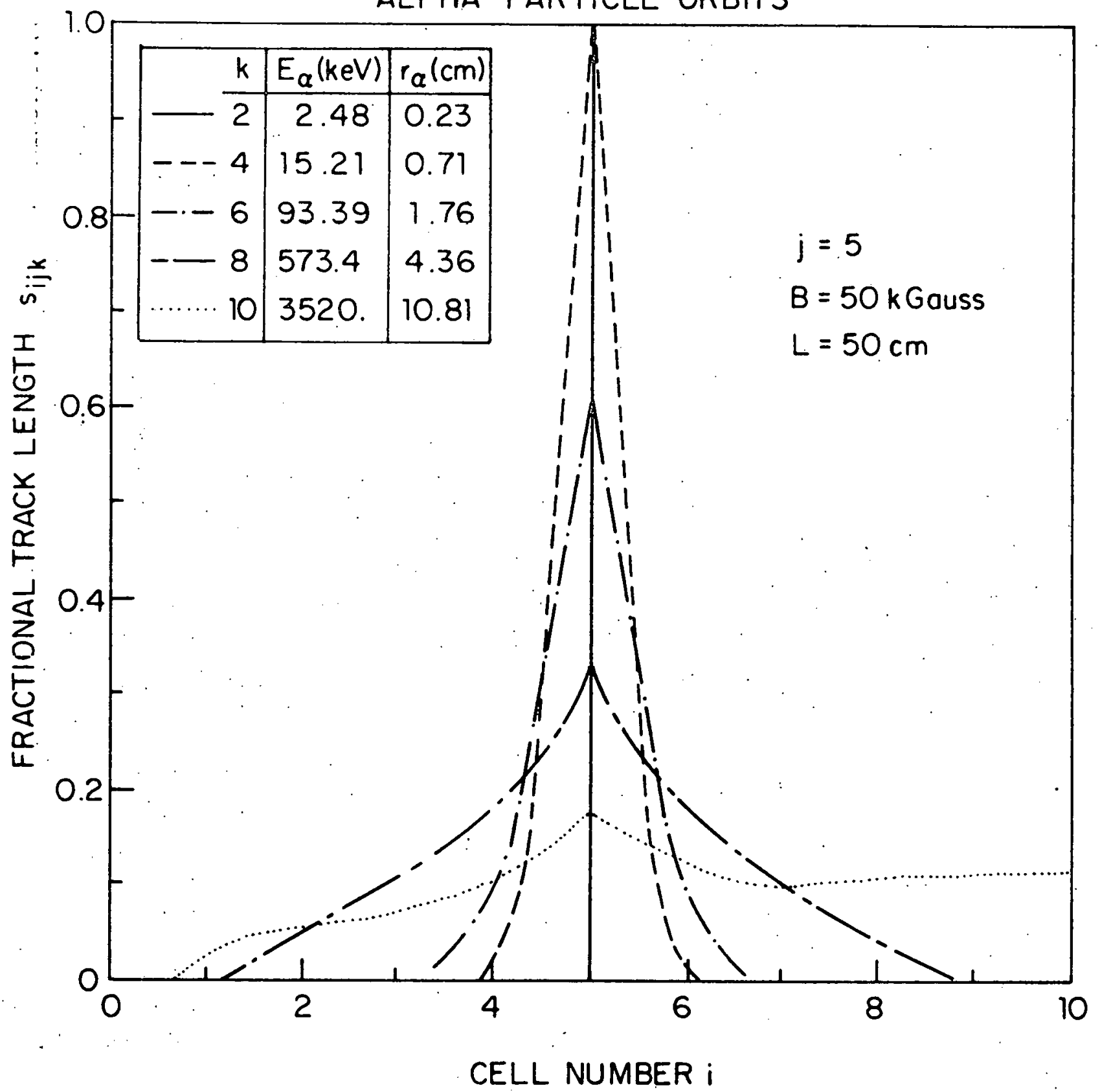

Figure 5 (appendix II) Fractional track length of an alpha particle born in cell $j$ which lies. in cell $i$ 
where $\delta n^{j k}$ is the number of alpha particles of energy $E_{\alpha}^{k}$ in cell $j$ and $\Delta E_{\alpha i, e}^{j k}$ is the energy lost by an alpha particle of energy $E_{\alpha}^{k}$ originating in cell $j$, given by

$$
\left.\Delta E_{\alpha i, e}^{j k}=\sum_{i} s_{i j k} \frac{d E_{\alpha}^{i k}}{d t}\right)_{i, e} \Delta t
$$

where $s_{i j k}$ is the fractional track length of an alpha particle of energy $E_{\alpha}^{k}$, starting in cell $j$, lying in cell $i$, and $\left.\frac{d E_{\alpha}{ }^{i k}}{d t}\right)_{i, e}$ is the energy loss rate of an alpha particle of energy $E_{\alpha}^{k}$ for the background plasma conditions of cell i.

The alpha particles whose orbits intersect the walls are assumed to lose their kinetic energy and to become part of the thermal alpha population in the cell immediately adjacent to the wall so that

$$
\begin{aligned}
& \delta n_{\alpha}^{j k}{ }^{j}=\left(1-s_{j k}^{w a l l}\right) \delta n_{\alpha}^{j k} \\
& n_{\alpha_{t h}}^{1} \cdot=n_{\alpha_{t h}}^{1}+\sum_{j} \sum_{k} \cdot s_{j k}^{w a l l} \delta n_{\alpha}^{j k}
\end{aligned}
$$

To preserve charge neutrality, electrons are assumed to be transported to the wall with these "lost" alpha particles. The electrons transported are moved one cell at a time from the source cell $j$ to the wall cell $i=1$, according to

$$
\begin{aligned}
& n_{e}^{\ell-1}=n_{e}^{\ell}-\sum_{k} 2 s_{j k}^{\text {wall }} \delta n_{\alpha}^{j k} \\
& n_{e}^{\ell-1}=n_{e}^{\ell-1}+\sum_{k} 2 s_{j k}^{\text {wall }} \delta n_{\alpha}^{j k}
\end{aligned}
$$


with the temperature rise in each cell due to the passage of these hotter interior electrons being given by

$$
T_{e}^{\ell-1},=T_{e}^{\ell-1}+\frac{\sum_{k} 2 s_{j k}^{w a l 1} \delta n_{\alpha}^{j k}}{n_{e}^{\ell-1}} T_{e}^{\ell}
$$

which allow the electrons to reach self-equilibration in each cell before being transported to the next cell.

The results of the one-dimensional problem of chapter 2 for no a]pha energy deposition and for purely local, $r_{\alpha} \rightarrow 0$, alpha energy deposition (where $s_{i j} \equiv \delta_{i j}$ and $s_{j}^{\text {wall }} \equiv 0$ for all $k$ ) are compared in figure 6 to those for the alpha energy deposition model described in this section. The center $(i=10)$ temperatures are plotted as a function of time, and the importance of the spatial transport of the alpha particles is very evident. In the absence of alpha particle heating, the plasma simply cools through radiation and thermal conduction losses. For purely local alpha particle energy deposition, where the alpha particles are perfectly contained in the spatial cell in which they were "born" $\left(r_{\alpha}<\Delta x\right)$, the electron and ion temperatures rise dramatically. The electrons have a higher temperature at early times because of the high ion thermal conduction losses, and the ions have a higher temperature at later times because radiation has become the dominant loss mechanism (although at earily times and low background plasma temperatures most of the alpha particle energy is deposited in the ion fluid, while at the high background temperatures existing at later times the predominant alpha particle energy loss is to the electrons). When the alpha particle 


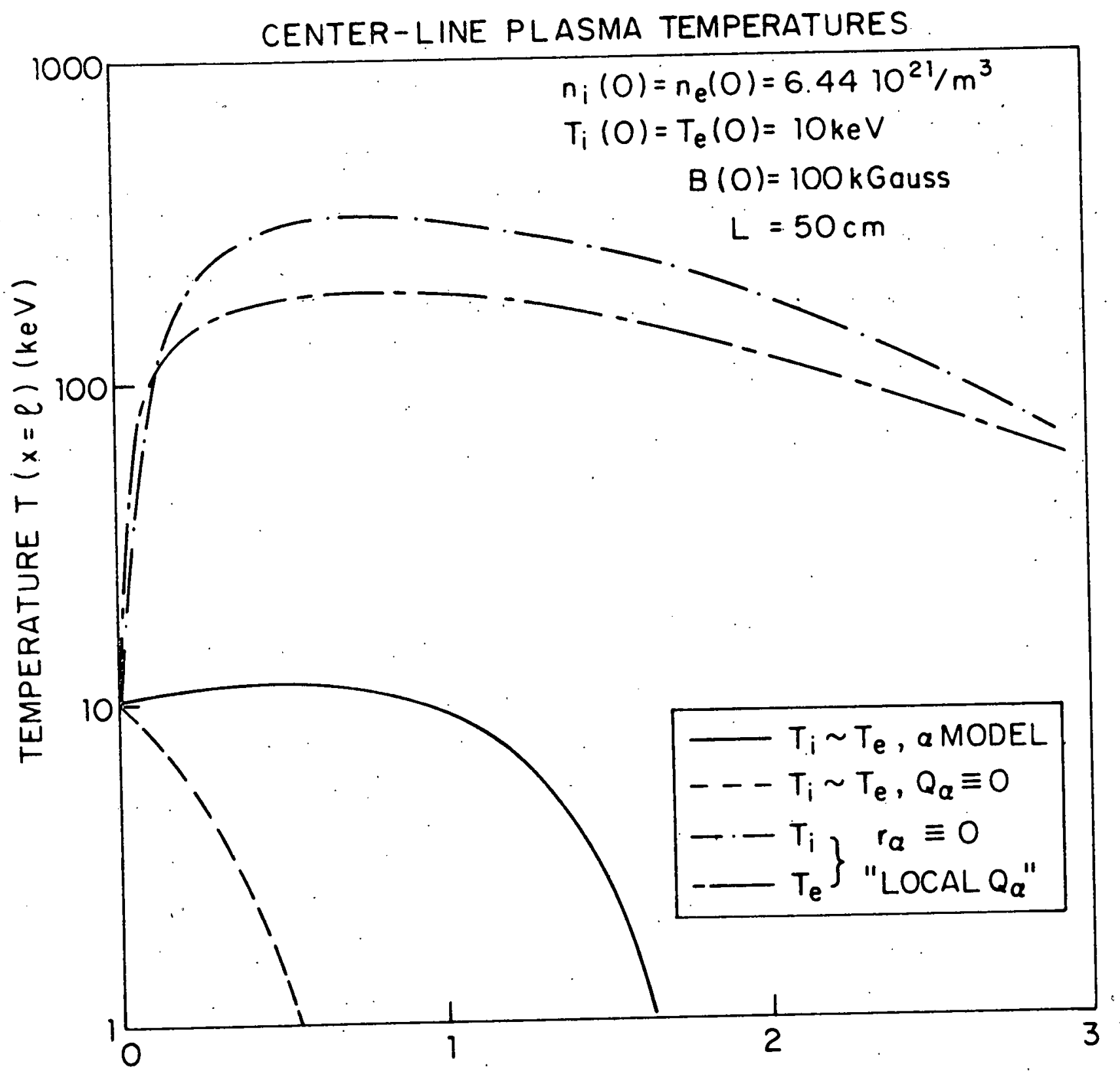

TIME + (sec)

Figure 6 (appendix II) Time evolution of the central $(x=\ell)$ plasma temperature for different models of the spatial dependence of alpha particle energy deposition 
gyroradii are modelled and their wall collisions taken into account, the energy deposited in the background by the alpha particles is greatly reduced since these high energy particles have large gyroradii in typical magnetic fields.

The fraction of alpha particles of various energies which are lost from a given point in the plasma through wall-collisions are shown in figures 7 and 8 for two different values of magnetic field. While low energy ( $\approx 10 \mathrm{keV}$ ) alpha particles are completely contained, higher energy $(\lambda 100 \mathrm{keV})$ particles often collide with the wall even when born well within the plasma. 


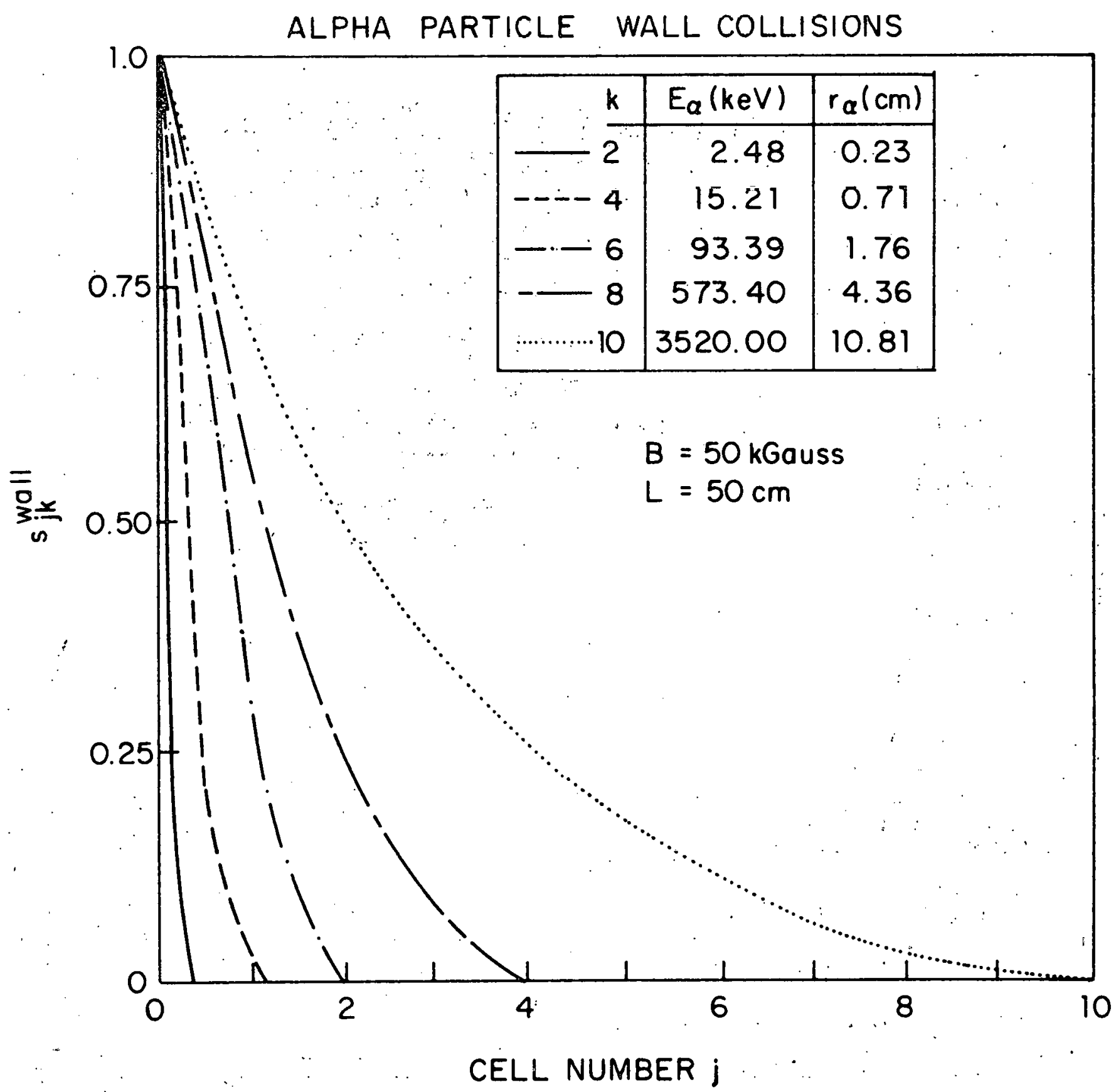

Figure 7. (appendix II) Fraction of alpha particles born in cell $j$ which collide with the wall 


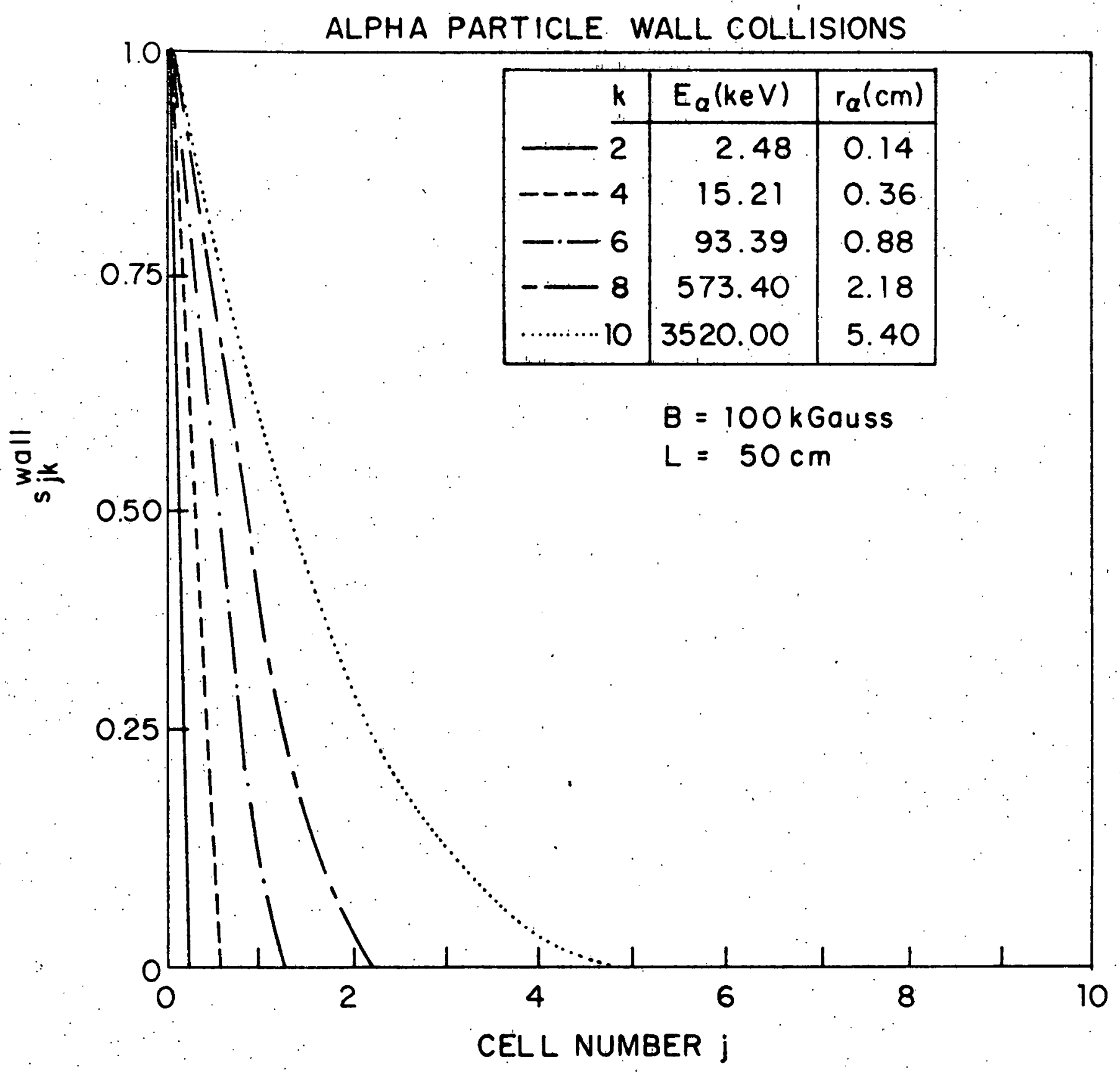

Figure 8 (appendix II) Fraction of alpha particles born in cell $j$ that collide with the wall 
Appendix III. Shock Heating - linear geometry

The power per unit cross-sectional area needed to shock-heat plasma to a desired temperature $T_{6}$, for initial imbedded magnetic field $B_{1}$, fill pressure $p_{1}$ and fill temperature $T_{1}=3000 \mathrm{~K}$, is found by solving a set of five simultaneous nonlinear al gebraic equations. The first three equations are derived from the shock jump equations [III-1], while the other two represent conservation of total energy and of magnetic flux during the re-expansion period [III-2]. Exact numerical results of specific power and energy inputs are compared to analytic expressions derived from the strong shock approximation [III-3, III-4], and the parametric dependence on initial state and final temperature is described.

The magnetohydrodynamic shock jump equations corresponding to conservation of mass, momentum and energy through the shock front are

$$
\begin{aligned}
\rho u \equiv m & =\text { constant } \\
m u+p+\frac{B^{2}}{2 \mu} & =\text { constant } \\
m\left(\frac{u^{2}}{2}+\frac{\gamma}{\gamma-1} \frac{p}{\rho}\right)+\frac{u B^{2}}{\mu} & =\text { constant }
\end{aligned}
$$

These three equations, which are written in the shock frame of reference, when combined with the equation for frozen magnetic flux

$$
\frac{B}{\rho}=\text { constant }
$$

can be rewritten for the incident shock as

$$
\frac{p_{2}}{p_{1}}=x_{1} \frac{n_{2} T_{2}}{n_{1} T_{1}}=\frac{n_{1}\left(x_{1}-1\right)^{3}+\left(\alpha x_{1}-1\right)}{\alpha-x_{1}}
$$




$$
\left.u_{1}=x_{1} u_{2}=a_{1} \sqrt{\frac{x_{1}}{\gamma}\left[\frac{\frac{p_{2}}{p_{1}}-1+\eta_{1}\left(x_{1}{ }^{3}-1\right)}{x_{1}-1}\right.}\right]
$$

and for the reflected shock as

$$
\begin{aligned}
& \frac{p_{2}}{p_{1}}=x_{2} \frac{n_{5} T_{5}}{n_{2} T_{2}}=\frac{n_{2}\left(x_{2}-1\right)^{3}+\left(\alpha x_{2}-1\right)}{\alpha-x_{2}} \\
& \dot{\tilde{u}}_{2}=x_{2} \tilde{u}_{5}=a_{2} \sqrt{\frac{x_{2}}{\gamma}\left[\frac{\frac{p_{5}}{p_{2}}-1+n_{2}\left(x_{2}^{3}-1\right)}{x_{2}-1}\right]}
\end{aligned}
$$

where

$$
\begin{aligned}
x_{1,2} & \equiv \frac{\rho_{2,5}}{\rho_{1,2}}=\frac{B_{2,5}}{B_{1,2}} \\
n_{1,2} & \equiv \frac{B_{1,2}^{2},}{2 \mu p_{1,2}} \\
\alpha & \equiv \frac{\gamma+1}{\gamma-1} \quad(\gamma \text { taken as } 5 / 3) \\
a_{1,2} & =\frac{/ \gamma \frac{p_{1,2}}{\rho_{1,2}}}{\gamma}
\end{aligned}
$$

The subscript 1 refers to the pre-shock gas, the subscript 2 refers to once-shocked plasma, and the subscript 5 refers to the twice-shocked plasma. Thus $u_{1}$ and $u_{2}$ are the upstream and downstream velocities with respect to the incident shock front, while $\tilde{u}_{2}$ and $\tilde{u}_{5}$ are the upstream and downstream velocities with respect to the reflected shock front.

These two sets of equations are related by the boundary conditions that both the initial pre-shock gas and the final twiceshocked plasma are quiescent in the laboratory frame of reference, so 
that

$$
\dot{u}_{2}-u_{1}=\tilde{u}_{2}-\tilde{u}_{5}
$$

Using equations (A III.6), (A III.8) and the relation

$$
\left(\frac{a_{2}}{a_{1}}\right)_{2}=\frac{p_{2}{ }^{\circ}}{p_{1} \rho_{2}}=\frac{1}{x_{1}} \frac{p_{2}}{p_{1}}
$$

this velocity condition can be rewritten to give

$$
\left(x_{1}-1\right)\left[\frac{p_{2}}{p_{1}}-1+n_{1}\left(x_{1}^{2}-1\right)\right]=\frac{p_{2}}{p_{1}}\left(1-\frac{1}{x_{2}}\right)\left[\frac{p_{1}}{p_{2}}-1+n_{2}\left(x_{2}^{2}-1\right)\right]
$$

The flux conservation equation for the re-expansion is

$$
B_{6}=B_{3}\left(1-\frac{\rho_{1}}{\rho_{5}}\right)+B_{5}\left(\frac{\rho_{1}}{\rho_{5}}\right)
$$

The subscripts 3 and 6 refer to the magnetic piston and to the final post-expansion plasma, and $\frac{\rho}{\rho_{5}}$ and $\left(1-\frac{\rho}{\rho_{5}}\right)$ are the fractional volumes of the twice-shocked plasma and of the magnetic piston. The equation for total energy conservation is similarly

$$
\frac{\mathrm{B}_{6}^{2}}{2 \mu}+\frac{3}{2} \mathrm{n}_{6} \mathrm{kT}_{6}=\frac{\mathrm{B}_{3}^{2}}{2 \mu}\left(1-\frac{\rho_{1}}{\rho_{5}}\right)+\left(\frac{\mathrm{B}_{5}{ }^{2}}{2 \mu}+\frac{3}{2 \mathrm{n}_{5}} \mathrm{kT}_{5}\right) \frac{\rho_{1}}{\rho_{5}}
$$

Equations (A III.5), (A III.7) and (A III.9), when all pressure ratios are re-expressed as temperature ratios and combined with the relation

$$
n_{5}=x_{2}^{n}=4 x_{1} x_{2}^{n}{ }_{1}
$$

(where the initial fill gas is assumed to be molecular DT which is completely ionized by the incident shock), are rewritten as 


$$
\begin{gathered}
4 x_{1} T_{2}\left(\alpha-x_{1}\right)=n_{1} T_{1}\left(x_{1}-1\right)^{3}+T_{1}\left(\alpha x_{1}-1\right) \\
4 x_{2} T_{5}\left(\alpha-x_{2}\right)=x_{1} n_{1} T_{1}\left(x_{2}-1\right)^{3}+4 T_{2}\left(\alpha x_{2}-1\right) \\
x_{2}\left(x_{1}-1\right)\left[4 x_{1} T_{2}-T_{1}+n_{1} T_{1}\left(x_{1}^{2}-1\right)\right]= \\
x_{1}\left(x_{2}-1\right)\left[4 x_{2} T_{5}-4 T_{2}+x_{1} n_{1} T_{1}\left(x_{2}^{2}-1\right)\right]
\end{gathered}
$$

Similarly, equations (A III.10) and (A III.11) when combined with the relation

$$
p_{3}=\frac{B_{3}^{2}}{2 \mu}=p_{2}+\frac{B_{2}^{2}}{2 \mu}
$$

can be rewritten to give

$$
\begin{gathered}
x_{2}{ }^{2}\left(B_{6}-B_{1}\right)^{2} x_{1} n_{1} T_{1}=B_{1}^{2}\left(x_{1} x_{2}-1\right)^{2}\left(x_{1} n_{1} T_{1}+4 T_{2}\right) \\
4 x_{1}\left(x_{1} x_{2}-1\right) B_{1}^{2} T_{2}+n_{1} T_{1}\left\{x_{1}{ }^{2} B_{1}{ }^{2}\left[x_{2}{ }^{2}+\left(x_{1} x_{2}-1\right)\right]-x_{1} x_{2} B_{6}{ }^{2}\right\}= \\
6 x_{1} x_{2} B_{1}{ }^{2}\left(T_{6}-T_{5}\right)
\end{gathered}
$$

The set of five simultaneous nonlinear equations formed by (A.III.12) through (A III.16) is solved for the five unknowns $x_{1}, x_{2}, T_{2} ; T_{5}$ and $B_{6}$ by an IMSL computer routine $[I I I-5]$ using Brown's methods [III-6, $[I I-7]$.

Identifying the incident shock speed as $u_{1}$, the reflected shock speed as $\tilde{u}_{2}$, and the speed of the magnetic piston as $u_{1}-\tilde{u}_{2}$, the specific power input required to drive the piston is given by

$$
P=p_{3}\left(u_{1}-\tilde{u}_{2}\right)=\left(p_{2}+\frac{B_{2}^{2}}{2 \mu}\right)\left(u_{1}-\tilde{u}_{2}\right)
$$


and the time required for the reflected shock to meet the magnetic piston (i.e. the tìme over whịch thịs specific power must be applied) is

$$
t=\frac{h}{u_{1}^{2}}\left(u_{1}+\tilde{i}_{2}\right)
$$

for a system of length $h$. Given an inner radius $r_{j}$ and an outer radius $r_{0}$, the total required energy input is therefore

$$
\left[=P A L=\left(p_{2}+\frac{B_{2}^{2}}{2 \mu}\right)\left(u_{1}-\tilde{u}_{2}\right) \pi\left(r_{0}^{2}-r_{i}^{2}\right) \frac{h}{u_{1}{ }^{2}}\left(u_{1}+\ddot{u}_{2}\right)\right.
$$

and the energy input per unit volume is size-independent and equal to

$$
e=\left(p_{2}+\frac{B_{2}^{2}}{2 \mu}\right)\left(1-\frac{\tilde{u}_{2}^{2}}{u_{1}^{2}}\right)
$$

The specific power input required to shock-heat a plasma to fusion grade ( $~ k \mathrm{keV}$ ) temperatures is shown in figures 1 and 2 , for various values of initial imbedded magnetic field as well as fill pressure (and hence density). The equations as formulated do not allow solutions for $B_{1}=0$, so $B_{1}=10$ gauss is chosen as a "negligible" initial field. The various plasma states calculated agree well with estimates from the strong-shock approximation $\left(M \equiv \frac{u}{a} \rightarrow \infty\right)$, allowing analytic study of the parametric dependence of required shock-heating energy input on initial conditions and desired final temperatures. The strong shock limits

$$
\begin{aligned}
& x_{1}=4 \\
& x_{2}=2.5 \\
& \frac{\tilde{u}_{2}}{u_{1}}=0.5
\end{aligned}
$$




\section{PRE-BURN CONDITIONS}

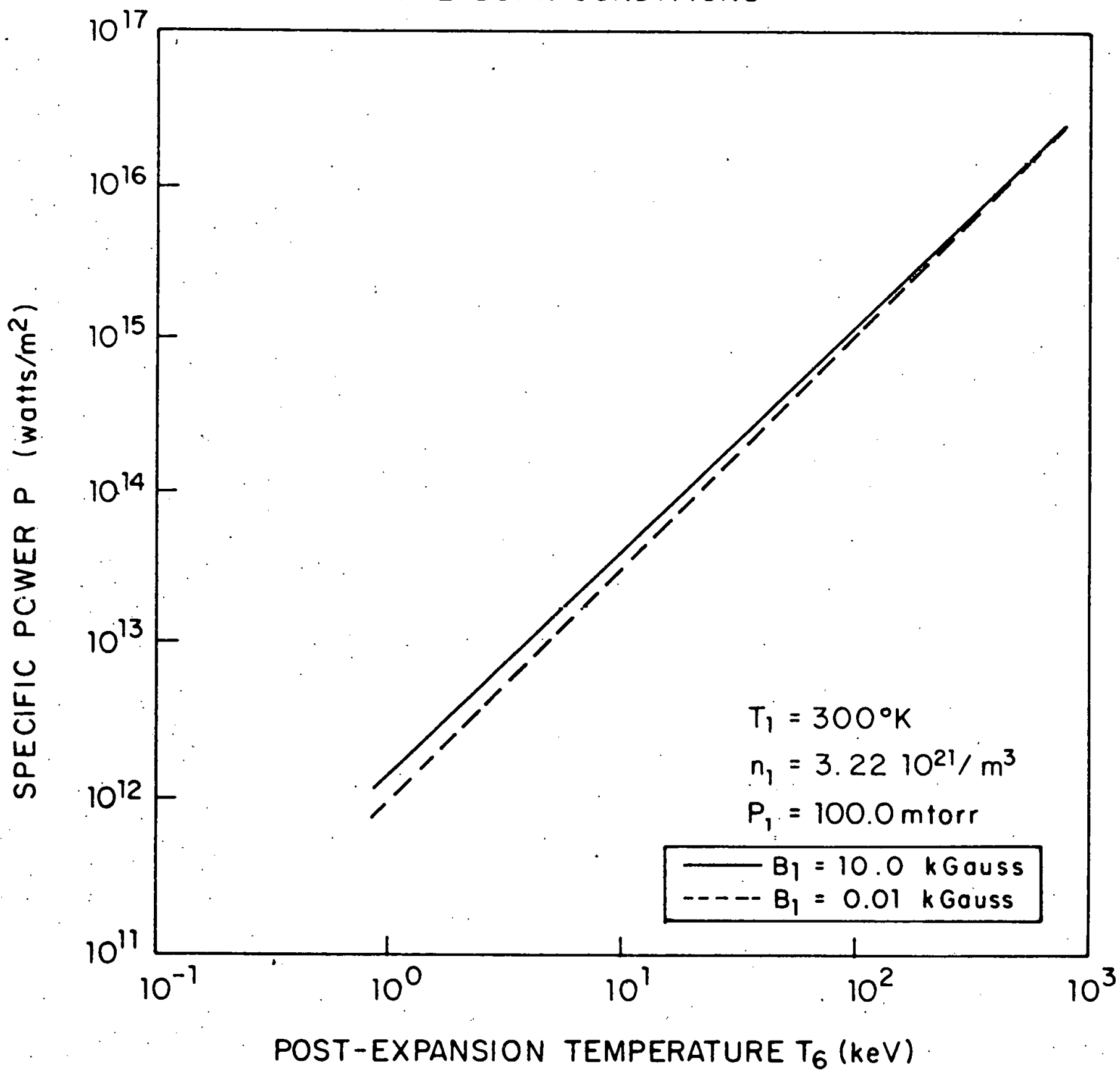

Figure 1 (appendix III) Power per unit area required to shock-heat to a final temperature $T_{6}$, for various values of the initial fill magnetic field 


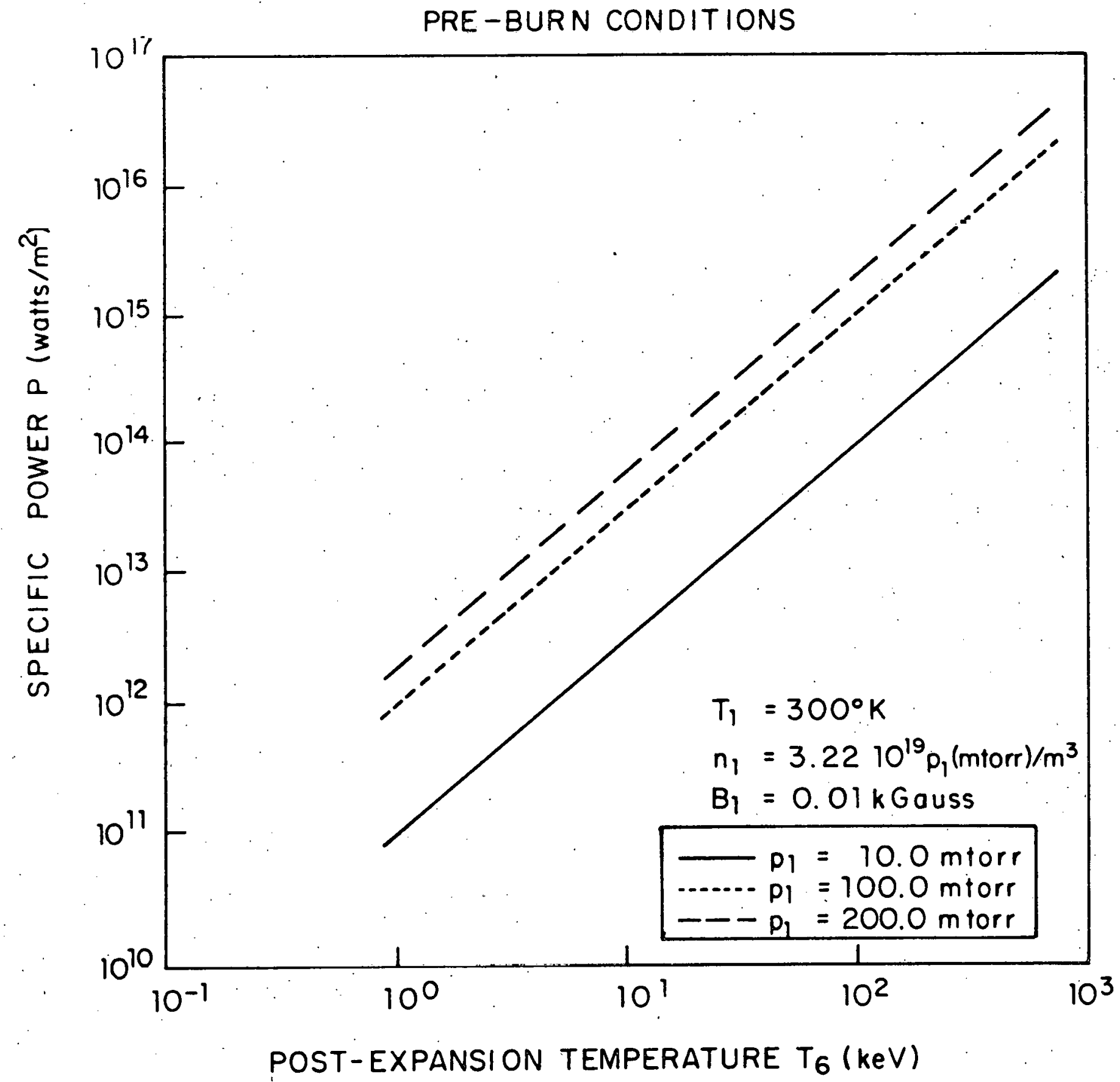

Figure 2 (appendix. III) Power per unit area required to shock-heat to a final temperature $T_{6}$, for various values of the initial fill pressure 


$$
\frac{T_{5}}{T_{2}}=2.4
$$

when combined with the assumption of small initial magnetic field (so that $\left.B_{1,2,5} \ll B_{3,6}\right)$ give the post-expansion state as

$$
\begin{aligned}
& \mathrm{B}_{6} \approx 0.9 \mathrm{~B}_{3} \\
& \mathrm{~T}_{6} \approx 1.15 \mathrm{~T}_{5}
\end{aligned}
$$

With equation (A III.21) relating the final desired post-expansion temperature to the shock-heating results, and using the strong shock condition

$$
\mathrm{T}_{2}\left({ }^{0} \mathrm{~K}\right) \sim 1.1410^{-5} \mathrm{u}_{1}^{2}(\mathrm{~m} / \mathrm{sec})
$$

the energy per unit volume required to heat to any desired temperature $T_{6}$ is approximately

$$
\begin{aligned}
& \mathrm{e}=\mathrm{p}_{3}\left(1-\frac{\tilde{\mathrm{u}}_{2}^{2}}{\mathrm{u}_{1}{ }^{2}}\right) \sim .75 \mathrm{p}_{2} \\
& \mathrm{e} \cdot\left(\frac{\mathrm{J}}{\mathrm{m}^{3}}\right) \stackrel{\sim}{=} 0^{7} \frac{\mathrm{p}_{1} \text { (mtorr) }}{\mathrm{T}_{1}\left({ }^{0} \mathrm{~K}\right)} \mathrm{T}_{6}(\mathrm{keV})
\end{aligned}
$$

and the associated power per unit area is

$$
\begin{aligned}
& P=p_{3}\left(u_{1}-\tilde{u}_{2}\right) \sim .5 p_{2} u_{1} \\
& P\left(\frac{\text { watts }}{m^{2}}\right) \cong 410^{12} \frac{p_{1} \text { (mtorr) }}{T_{1}\left(\cdot{ }^{0} \mathrm{~K}\right)} T_{6}^{3 / 2}(\mathrm{keV})
\end{aligned}
$$

Finally, the magnetic piston field required is given by

$$
\mathrm{B}_{3} 2=\mathrm{B}_{2}^{2}+2 \mu \mathrm{p}_{2} \sim 2 \mu \mathrm{p}_{2}
$$




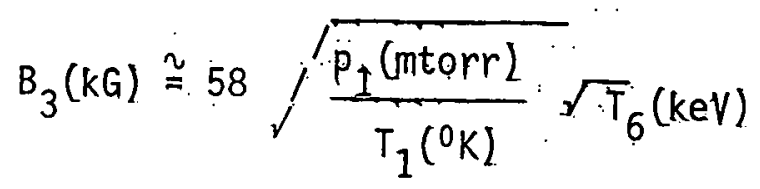

A comparison of these approximate expressions with the calculated exact results for various values of $T_{6}$, for $p_{1}=100$ mtorr, $T_{1}=3000 \mathrm{~K}$ (so that $n_{1}=3.2210^{+21} / \mathrm{m}^{3}$ ) and $B_{1}=0.01$ kGauss, is given in the following table:

Approximate Fxart Approximate Exact

\begin{tabular}{llllll}
$T_{6}(\mathrm{keV})$ & $P\left(\frac{\text { watts }}{\mathrm{m}^{2}}\right)$ & $P\left(\frac{\text { watts }}{\mathrm{m}^{2}}\right)$ & $\mathrm{e}\left(\frac{\mathrm{j}}{\mathrm{m}^{3}}\right)$ & $\mathrm{e}\left(\frac{\mathrm{J}}{\mathrm{m}^{3}}\right)$ \\
\hline 2.1644 & $4.2510^{12}$ & $2.9410^{12}$ & $7.2110^{6}$ & $6.3710^{6}$ \\
3.4304 & $8.4710^{12}$ & $5.8710^{12}$ & $1.1410^{7}$ & $1.0110^{7}$ \\
5.4368 & $1.69 \cdot 10^{13}$ & $1.1710^{13}$ & $1.8110^{7}$ & $1.5910^{7}$ \\
8.6168 & $3.3710^{13}$ & $2.34 \cdot 10^{13}$ & $2.8710^{7}$ & $2.5510^{7}$ \\
13.657 & $6.7310^{13}$ & $4.6610^{13}$ & $4.5510^{7}$ & $4.0310^{7}$ \\
21.644 & $1.3410^{14}$ & $9.3010^{13}$ & $7.2110^{7}$ & $6.3710^{7}$ \\
34.304 & $2.6810^{14}$ & $1.8610^{14}$ & $1.1410^{8}$ & $1.01 .10^{8}$ \\
54.368 & $5.3510^{14}$ & $3.7010^{14}$ & $1.8110^{8}$ & $1.5910^{8}$ \\
86.168 & $1.0710^{15}$ & $7.3810^{14}$ & $2.8710^{8}$ & $2.5510^{8}$
\end{tabular}

The functional dependence on $T_{6}$ and $p_{1}$ is predicted exactly. The absolute value of the magnetic piston field is conservatively overestimated by an almost constant 19\%, and the specific energy input and the power per unit area by $12.5 \%$ and $44 \%$, respectively. 
Appendix IV: System Parameters

The results of the cycle calculation are summarized in the following table for two potentially interesting reactor designs (with reasonable energy multiplications of 10-20) and a breakeven experiment (with an energy gain of 2-3). In these cases, classical thermal conductivities are used and there is zero alpha particle heating.

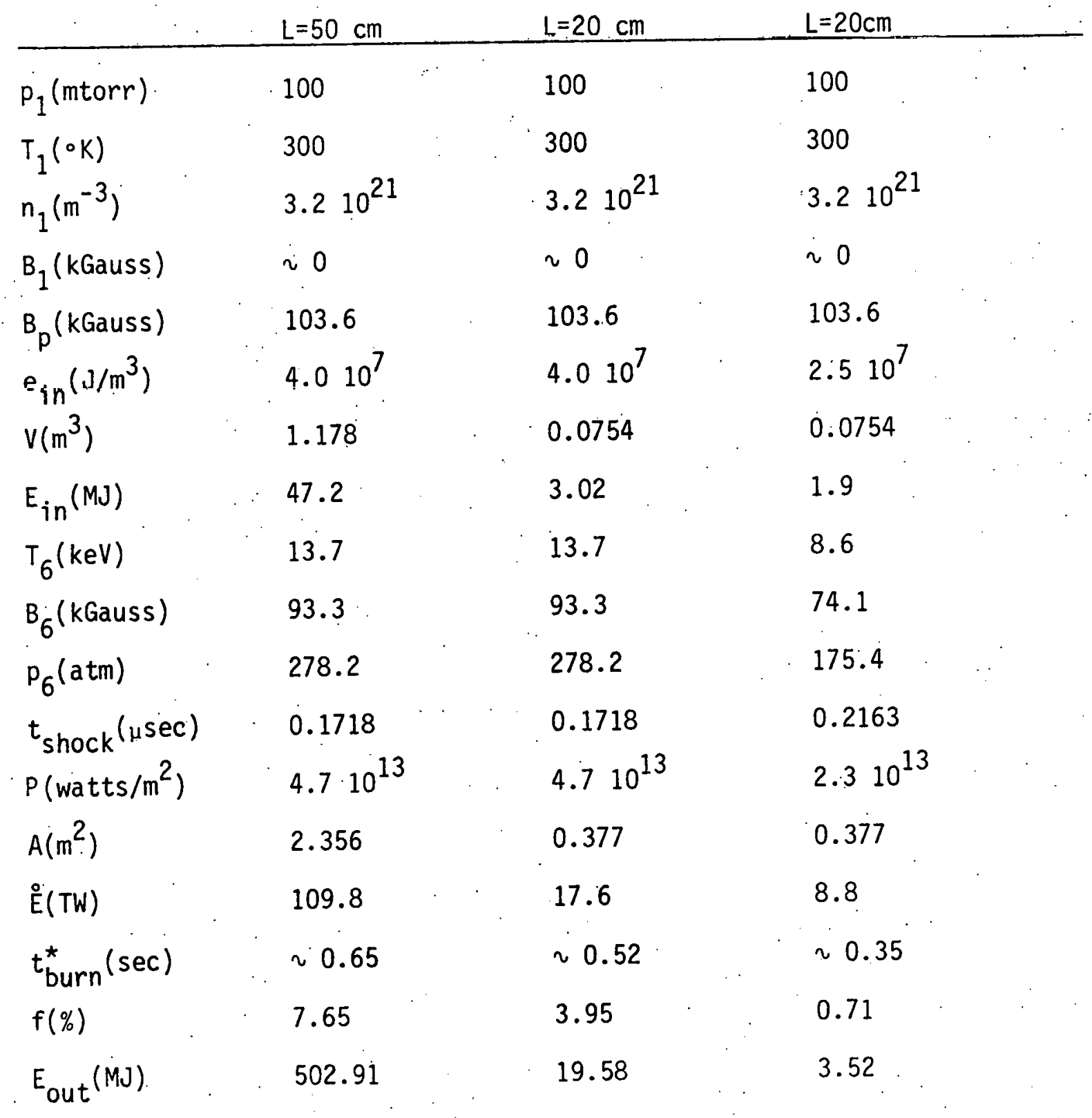




\begin{tabular}{lrrr} 
& $L=50 \mathrm{~cm}$ & $L=20 \mathrm{~cm}$ & $L=20 \mathrm{~cm}$ \\
\hline$Q$ & 13.56 & 7.49 & 2.85 \\
$q_{\alpha}^{\dagger}\left(\mathrm{J} / \mathrm{m}^{2}\right)$ & $1.310^{7}$ & $2.610^{6}$ & $4.710^{5}$ \\
$q_{\mathrm{th}}^{\dagger}\left(\mathrm{J} / \mathrm{m}^{2}\right)$ & $5.010^{6}$ & $2.010^{6}$ & $1.310^{6}$ \\
$\mathrm{q}_{n}^{\pi}\left(\mathrm{J} / \mathrm{m}^{2}\right)$ & $5.010^{7}$ & $1.010^{7}$ & $1.910^{6}$
\end{tabular}

" $t_{\text {burn }}$ is the time at which all plasma is at a temperature below $1 \mathrm{keV}$. ${ }^{+} q_{\alpha}$ is the total alpha energy impacting on walls $\ddagger^{q_{\text {th }}}$ is the total thermal impact on the walls ${ } q_{n}$ is the total neutron energy flux impacting on walls 
Appendix V: Nomenclature

A Area of dielectric end wall where magnetic piston is formed (appendix III)

a Plasma sound velocity (appendix III)

$B \quad$ Transverse magnetic field $B_{Z}$

$c_{m} \quad$ Plasma magnetosonic velocity (chapter 2)

E. Energy

e Electronic charge; energy per unit volume

$f$ Burn fraction (chapter 3); fraction of alpha particle energy deposited to ions (appendix II)

$f_{1}$ Ratio of reactor inner radius to height

$f_{2}$ Ratio of reactor outer radius to height

h Reactor height

j Transverse current density $j_{y}$ (chapter 2).

k Boltzmann constant

L. Distance between walls

\& Characteristic length; alpha particle track length (appendix II)

m : Particle mass

n Number density

P Fluid momentum (chapter 2); power per unit area driving shock (appendix. III)

p Pressure (kinetic)

$\mathrm{P}_{\text {wall }}$ System pressure (kinetic and magnetic)

Q Rate of change of energy (chapter 2); energy multiplication (chapter 3)

$q$ : Energy per unit area 
Radius

$\$ \quad$ Particle source function (chapter 2)

s Fractional alpha particle track length (appendix. II)

$T$ Temperature

$t \quad$ Time

u,v Velocity

$V \quad$ Relative cell volume (chapter 2)

W Random number (appendix II)

$X$ Cell boundary

$\bar{X} \quad$ cell center

x. Shock compression ratio (appendix III)

y Cell compressibility (chapter 2)

Z Atomic charge

Greek

$\gamma \quad$ Ratio of heat capacities

$n$ Transverse electrical resistivity (chapter 2)

$\theta$ Measure of implicitness of difference scheme

k Transverse thermal conductivity

$\lambda \quad$ Absorption length

$\rho \quad$ Mass density

$\sigma \quad$ Cross-section

$\tau \quad$ Characteristic time

$\xi \quad$ Lagrangian variable

en . Coulomb logarithm 
Subscripts

\begin{tabular}{|c|c|c|c|c|}
\hline$b, B$ & & Bremsstrahlung ra & adiation & \\
\hline$c$ & & Self-collision; & thermal & conduction \\
\hline cl & & Classical & & \\
\hline D & & Deuteron; Debye; & ; drift & \\
\hline e & & Electron & & \\
\hline$f$ & & Fusion & & \\
\hline$g$ & & Gyro-orbit & $\cdots$ & \\
\hline$j$ & & Ion & & . \\
\hline in & & Input; initial & . . . & \\
\hline 了 & & Joule heating & & \\
\hline$n$ & & Neutron & & \\
\hline 0 & & Reference & & \\
\hline $\mathrm{p}$ & & Photon & $\because$ & \\
\hline $\mathrm{R}$ & & DT reaction & $\cdot$ & \\
\hline$s$ & & Shock: particle & species & \\
\hline $\mathbf{T}$ & & Triton & & \\
\hline$x$ & & Exchange & . & . \\
\hline$z$ & & Atomic charge & . & \\
\hline$\alpha$ & & Alpha particle & & \\
\hline
\end{tabular}


REFERENCES

\section{Chapter 1}

1. G.I. Budker, 6th European Conf., Controlled Fusion and Plasma Physics, Moscow; 2, 136. (1973).

2. C.M. Braams et al., Proc. 5th International Conf. Controlled Fusion and Plasma Physics, IAEA, Tokyo, Vol. II, 725 (1975).

3. B. l,ehnert, Proc. 5th Interiational Conf. Controlled Fusion and Plasma Physics, IAEA, Tokyo, Vol. II, 725 (197.5).

4. A.R. Sherwood, B.L. Freeman, R.A. Gerwin, T.R. Jarboe, R.A. Krakowski,.R.C. Malone, J. Marshall, R.L. Miller and B. Suydam; Los Alamos Scientific Laboratory Report LA-6707-P (1977).

5. R.A. Gross, Nucl. Fusion, 13, 293 (1973).

6. R.A. Gross, Nucl. Fusion, 15, 729 (1975).

7. R.A. Gross, Y.G. Chen, E. Halmoy, and P. Moriette, Phys. Rev. Letters, 25, 575 (1970).

8. E. Halmoy, Phys. Fluids 14, 2134 (1971).

9. P. Moriette, Phys. Fluids 15, 51 (1971).

10. Y.G. Chen, C.K. Chu, R.A. Gross, E. Halmoy, P. Moriette and S. Scheider; "Plasma Heating by Strong Shock Waves", Proc. 4th International IAEA Conf. Plasma Physics and Controlled Nuclear Fusion Research, Madison, Wisconsin, III, 169 (1971).

11. R.A. Gross, "Shock Wave Heating to Fusion Temperatures", Proc. of 9th International Shock Tube Symposium, Recent Developments on Shock Tube Research: Ed's Bershader and Griffith; Stanford University Press; 72 (1973).

12. H.S. Carslaw and J.C. Jaeger, Conduction of Heat in Solids, Oxford University Press (1959).

13. E.R.G. Eckert and R.M. Drake Jr., Heat and Mass Transfer, McGraw-Hi11 (1959),

14. M. Mitchner and C.H. Kruger Jr., Partially Ionized Gases, Wiley (1973).

15. M.S. Chu., Phys. Fluids 16, 1441 (1973). 
16. G.E. Vekshtein, D.D. Ryutov, M.D. Spektor and P.Z. Chebotaev, Zh. PrikT. Mekh. Tekh. Fiz. 15, 731 (1974) [J. Appl. Mech. Tech. Phys. 15, 731 (1974)].

17. B.K. Jensen, Phys. Fluids 20, 373 (1977).

18. I.R. Lindemuth, J.S: Pettibone, J.C. Stevens, R.C. Harding, D.M. Kraybil1 and L.J. Suter, Phys. Fluids 21, 1723 (1978).

19. M.S. Chu, Phys. Fluids, 15, 413 (1972).

20. S.T. Butler and M.J. Buckingham, Phys. Rev. 126, 1 (1962).

21. R. Conn, UWFDM-10, University of Wisconsin (1972).

22. D.J. Sigmar and G. Joyce, Nucl. Fusion 11, 447 (1971).

23. D.J. Rose, Nucl. Fusion 9 , 183 (1969).

24. T. Kammash, Fusion Reactor Physics: Principles and Technology, Ann Arbor Science (1975).

25. H.J. Willenberg, Nucl. Fusion 19, 313 (1979).

26. R.A. Gross, Columbia University internal memorandum (1972).

27. J. Benford, V. Bailey, D. Oliver, M.D. Capua and R. Cooper, PIFR-863-1, Physics International (1977).

28. L. Spitzer Jr., Physics of Fully Ionized Gases, Intersecience (1962).

\section{Chapter 2}

1. T.J.M. Boyd and J.J. Sanderson, Plasma Dynamics, Barnes and Noble (1969), p.: 51 .

2. Ibid, p. 53.

3. L.A. Artsimovich, Controlled Thermonuclear Reactions, Gordon and Breach Science Pub7ishers (1964), p. 82.

4. R.L. Liboff, Introduction to Theory of Kinetic Equations, Wiley (1969).

5. S.R. Seshadri, Fundamentals of Plasma Physics, American Elsevier Publishing Company (1973), p. 82.

6. C.F. Wandel, T. Hesselberg-Jensen and 0 . Kofoed-Hansen, Nucl. Instruments $\underline{4}, 269$ (1959): 
7. F. Reif, Fundamentals of Statistical and Thermal Physics, McGrawHill (1965), p. 515 .

8. W.F. Sommer, private communication.

9. B. Feinberg, Plasma Physics 18, 265 (1976).

10. L. Spitzer, Physics of Fülly Ionized Gases, Interscience (1962).

11. G. Hopkins, Symp. Tech. Cont. Thermonuclear Fusion Exps and the Eng. Aspects of Fusion Reactors, Austin, Texas, p. 795 (1972).

12. I.R. Lindemuth, J. Comp. Physics 25, 104 (1977).

13. T.A. 0liphant, G.E. Gryczkowski and T. Kammash, Nuclear Fusion 16, $263(1976)$.

14. T.A. 01iphant, private communication.

15. R.C. Richtmeyer and K.W. Morton, Difference Methods for InitialValue Problems, Interscience (1967), p. 198.

16. IMSL-International Mathematical and Statistical Libraries; Inc., Houston, Texas.

17. T.E. Hull, W.H. Enright and K.R. Jackson, TR 100, Department of Computer Science, University of Toronto (1976).

18. K.R. Jackson, W:H. Enright and T.E. Hul1, TR 101, Department of Computer Science, University of Toronto (1977).

19. B.K. Jensen, Columbia University Plasma Laboratory Report, No. $67(1976)$.

20. K.M. Brown, SIAM Journal on Numerical Analysis 6, 560 (1969).

21. K.M. Brown and J.E. Dennis Jr., Yale University Department of Computer Science Technical Report, p. 71 (1971).

22. M.S. Chü, Phys. Fluids 16, 1441 (1973).

23. B.K. Jensen, Phys. Fluids 20, 373 (1977).

24. I.R. Lindemuth, J.S. Pettibone, J.C. Stevens, R.C. Harding, D.M. Kraybil1 and L.J. Suter, UCRL- 7983L, Lawrence Livermore Laboratory (1977).

25. I.R. Lindemuth, J.S. Pettibone, J.C. Stevens, R.C. Harding, D.M. Kraybill and L.J. Suter, Phys. Fluids 21, 1723 (1978).

26. I.R. Lindemuth, private communication. 


\section{Chapter 4}

1. B. Lehnert, Proc. 5th International Conf. Controlled Fusion and

Plasma Physics, IAEA, Tokyo, Vol. II, p. 717 (1975).

2. G.E. Vekshtein, D.D. Ryutov and P.Z. Chabotaev, Physics of Plasma (USSR) 3 , 401 (1975).

3. B.K. Jensen, Phys. Fluids 20, 373 (1977).

4. I.R. Lindemuth, J.S. Pettibone, J.C. Stevens, R.C. Harding, D.M. Kraybill and L.J. Suter, Phys. Fluids 21, 1723 (1978).

\section{Appendix I}

1. L. Spitzer, Physics of Fully Ionized Gàses, Interscience (1962).

2. T. Kammash, Fusion Reactor Physics: Principles and Technology, Ann Arbor Science (1975).

3. N.A. Krall and A.W. Trivelpiece, Principles of Plasma Physics, McGraw-Hill (1973).

4. R.A. Gross, Comments Plasma Phys. 2, 155 (1976).

5. S. Glasstone and R.H. Lovberg, Controlled Thermonuclear Fusion, D. Van Nostrand (1960).

\section{Appendix II}

1. I.P Shkarofsky, T.W. Johnston and M.P. Bachynski, The Particle Kinetics of Plasmas, Addison-Wesley (1966).

2. S.T. Butler and M.J. Buchingham, Phys. Rev. 126, 1 (1962).

3. T. Kammash, Fusion Reactor. Physics: Principles and Technology, Ann Arbor Science (1975).

4. D.J. Sigmar and G. Joyce, Nucl. Fusion 11, 447 (1971).

5. Kammash, op. cit.

6. R. Conn, UWFCM-10, University of Wiscons in (1972). 


\section{Appendix III}

1. C.K. Chu and R.A. Gross, "Shock Waves in P.lasma Physics," Advances in Plasma Physics, Vol. 2, edited by Simon and Thompson, p. 139 (1969).

2. R.A. Gross, private combiunication.

3. R.A. Gross, Rev. Mod. Phys. 37, 724 (1965).

4. A.G. Gaydon and I:R. Hurle, The Shock Tube in High Temperature Chemical Physics, Reinhold (1963).

5. IMSL-International Mathematical and Statistical Libraries, Inc., Houston, Texas.

6. K.M. Brown, SIAM Journal on Numerical Analys is $\underline{6}, 560$ (1969).

7. K.M. Brown and J.E. Dennis Jr., Yale University Department of Computer Science Technical Report, p. 71 (1971). 


\section{LIST OF FIGURES}

\section{Chapter 1}

1. Schematic of a shock-heated wall-confined fusion reactor

2. Shock heating and re-expansion

3. Characteristic times for a plasma with temperature $T_{6}$, confined between walls $50 \mathrm{~cm}$ apart

\section{Chapter 2}

1. Reaction probabilities for several thermonuclear reactions in a deuterium-tritium plasma

2. Coordinate system for a one-dimensional plasma-wail study

3. Spacial zoning for the geometry shown in figure 2

4. Curve-fit used for the reaction probability

5. Spatial profiles of the time evolution of the plasma temperature

6. Spatial profiles of the time evolution of the total alpha particle number density (thermalized and nonthermalized)

7. Spatial profiles of the time evolution of the deuteron and triton number densities (with $n_{D}=n_{T}$ ), which together with the thermalized alpha particles form the ion fluid

8. Spatial profiles of the time evolution of the relative cell volumes

9. Spatial profiles of the time evolution of the transverse imbedded magnetic field

10. Spatial profiles of the time evolution of the induced electric currents

11. Spatial profiles of the time evolution of the bulk plasma velocity, 
where negative values correspond to flow toward the walls and positive values to flow away from the walls

12. Time evolution of the total system pressure (kinetic and magnetic) for various wáll separalion distances

13. Spatial profiles of the time evolution of the plasma temperature

14. Spatial profiles of the time evolution of the total alpha particle number density (thermalized and nonthermalized)

15. Spatial profiles of the time evolution of the plasma temperature

16. Spatial profiles of the time evolution of the total alpha particle number density (thermalized and nonthermalized)

\section{Chapter 3}

1. Limiting energy multiplication factors for a shock-heated wallconfined fusion reactor

2. Integration volume for a one-dimensional profile in the coaxial reaction chamber

3. Energy multiplication factors calculated from the one-dimensional results of chapter 2

4. Energy multiplication factors for a small $(L=20 \mathrm{~cm})$ system after various two-dimensional loss mechanisms have been taken into account

5. Energy multiplication factors for an intermediate $(L=50 \mathrm{~cm})$ system after various two-dimensional loss mechanisms have been taken into account.

6. Energy multiplication factors for a large $(L=1 \mathrm{~m})$ system after various two-dimensional loss mechanisms have been taken into account 


\section{Appendix II}

1. Time evolution of the ion temperature for different models of the time dependence of alpha particle energy deposition

2. Time evolution of the electron temperature for different models of the time dependence of alpha particle energy deposition

3. Alpha particle orbits which (a) intersect the wall, (b) are perfectly contained, or (c) are contained, but pass through the symmetry plane

4. Fractional track length of an alpha particle born in cell $j$ which lies in cell $\mathrm{i}$

5. Fractional track length of an alpha particle born in cell $j$ which lies in cell $i$

6. Time evolution of the central $(x=l)$ plasma temperature for different models of the spatial dependence of alpha particle energy deposition

7. Fraction of alpha particles born in cell $j$ which collide with the wall .

8. Fraction of alpha particles born in cell $j$ which collide with the wall

\section{Appendix III}

1. Power per unit area required to shock-heat to a final temperature $T_{6}$, for various values of the initial fill magnetic field

2. Power per unit area required to shock-heat to a final temperature $T_{6}$, for various values of the initial fill pressure 